\title{
SISTEMAS \\ DIFERENCIALES-ALGEBRAICOS: \\ APLICACIONES A PERTURBACIÓN SINGULAR Y CONTROL
}

\author{
María del Rosario Etchechoury \\ Tesis para acceder al grado de Doctor en Matemática
}

Dto de Matemática, Facultad de Ciencias Exactas, U.N.L.P.,

LEICI, Facultad de Ingeniería, U.N.L.P.,

junio 2001. 
A mi familia 
Agradecimientos:

A mis directores, Carlos Muravchik y María Amelia Muschietti, que me apoyaron y guiaron para realizar esta tesis.

A mis compañeros del Laboratorio de Electrónica Industrial, Control e Instrumentación de la Facultad de Ingeniería por hacer de mi lugar de trabajo un espacio agradable y cálido.

A mis compañeros del Departamento de Matemática de la Facultad de Ciencias Exactas que me alentaron a lo largo de este proyecto.

A mis amigos, que como siempre estuvieron conmigo y me incentivaron para emprender y culminar esta tesis.

A mis padres y esposo, que en todo momento, desde mis comienzos como estudiante de la Licenciatura en Matemática, comprendieron mi pasión por mi trabajo y nunca reclamaron el tiempo que no pude brindarles en muchas ocasiones.

A mis hijos, Ramiro y Leticia, que casi sin saberlo renuevan en mí cada día mis energías y mis fuerzas, indispensables para continuar en este camino.... 


\section{Índice general}

1. Introducción 5

1.1. Generalidades ..................... 5

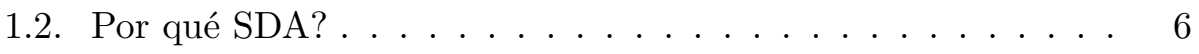

1.3. Distintas clases de SDA . . . . . . . . . . . . 7

1.4. Aplicaciones . . . . . . . . . . . . . . . . 9

1.5. Esquema general . . . . . . . . . . . . . . . . 11

2. Preliminares 13

3. SDA Semiexplícitos 15

3.1. Regularidad de SDAS . . . . . . . . . . . 16

3.2. Campo vectorial y espacio de configuración . . . . . . . 20

3.3. SDO con restricciones algebraicas . . . . . . . . . . 32

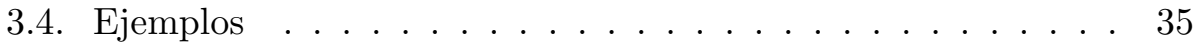

4. Sistemas con Perturbación Singular 39

4.1. Introducción . . . . . . . . . . . . . . . . . . 39

4.2. SPS en forma standard . . . . . . . . . . . . . . 43

4.3. SPS no-standard . . . . . . . . . . . . . . . 47

4.4. Aproximación de trayectorias . . . . . . . . . . . 55 
5. SDAS: un problema de control 73

5.1. Introducción . . . . . . . . . . . . . 73

5.2. Caso $m=1 \ldots \ldots \ldots \ldots \ldots \ldots$

5.3. Caso $m>1 \ldots \ldots \ldots \ldots \ldots$. . . . . . . . . . . . 82

5.4. Dinámica cero sin grado relativo vectorial . . . . . . . . 91

$\begin{array}{ll}\text { 6. Conclusiones } & 97\end{array}$

6.1. Resumen de las contribuciones . . . . . . . . . . . 97

6.2. Futuras investigaciones . . . . . . . . . . . . . . . . . 99

A. Teoría de la Variedad Centro 101

$\begin{array}{ll}\text { Bibliografía } & 105\end{array}$ 


\section{Capítulo 1}

\section{Introducción}

\subsection{Generalidades}

Los Sistemas Diferenciales Algebraicos (SDA) son también conocidos como sistemas singulares, implícitos, descriptores o sistemas generalizados. Surgen naturalmente como modelos dinámicos de aplicaciones de la ingeniería (tales como redes de circuitos eléctricos [1], sistemas de potencia [2], sistemas mecánicos con restricciones [3], ingeniería aeroespacial [4] y procesos químicos [5]); se utilizan también para modelar sistemas sociales, sistemas económicos, sistemas biológicos; etc.

En muchos casos los SDA pueden resolverse eficientemente por medio de métodos numéricos standard utilizados para la resolución de Sistemas Diferenciales Ordinarios (SDO). Este enfoque fue introducido por Gear [6], y utilizado por diferentes autores, por ejemplo en [7] y [8]. Sin embargo, los SDA suelen tener algunas propiedades que provocan que estos métodos numéricos fracasen. En [7] y [9], por ejemplo, se presentan algunos resultados acerca de las causas de tales dificultades para el caso particular de una clase de SDA lineales. Las técnicas utilizadas en estos trabajos son de naturaleza 
algebraica, y no brindan una información completa acerca de la existencia y unicidad de soluciones.

Otro enfoque diferente surge al considerar un SDA como un conjunto de ecuaciones diferenciales sobre una variedad. Esta aproximación geométrica permite desarrollar una teoría de existencia y unicidad de soluciones que da lugar a conocer nuevas propiedades de los SDA, y a analizar cuales son las causas por la que los métodos numéricos fallan algunas veces, [10].

\subsection{Por qué SDA?}

Consideremos un sistema diferencial de la forma,

$$
F(t, x(t), \dot{x}(t))=0
$$

donde $F: \mathbb{R} \times \mathbb{R}^{n} \times \mathbb{R}^{n} \rightarrow \mathbb{R}^{n}$ es una aplicación suave. Si la derivada parcial $D_{p} F(t, x, p)$ tiene rango constante $n$ sobre el dominio (abierto) de $F$ ó sobre algún entorno de $F^{-1}(0)$ en $\mathbb{R} \times \mathbb{R}^{n} \times \mathbb{R}^{n}$, entonces aplicando el Teorema de la Función Implícita (1.1) se puede escribir como,

$$
\dot{x}(t)=f(t, x(t))
$$

El sistema (1.1) representa un SDA cuando

$$
r g\left(D_{p} F(t, x, p)\right)=\rho<n,
$$

sobre el dominio de $F$ ó localmente sobre algún entorno de $F^{-1}(0)$.

En la actualidad las diferencias entre un SDA y un SDO están bien establecidas. En particular, un concepto que es comunmente usado para dar una medida de tales diferencias es el de índice (diferencial). Básicamente, el índice de un SDA es el número mínimo de diferenciaciones requeridas 
para obtener un SDO equivalente. Precisaremos este concepto en el Capítulo siguiente. Los SDA de índice $>1$ son conocidos como de alto índice y han sido ampliamente estudiados en las últimas décadas; por ejemplo en [11, 12] desde el punto de vista numérico y en [13] analizando una clase particular: los SDA lineales y con control.

\subsection{Distintas clases de SDA}

En esta Sección mencionaremos algunas clases de SDA, que aparecerán en los capítulos siguientes.

(i) Sistemas Diferenciales Algebraicos Semiexplícitos (SDAS)

Son sistemas de la forma

$$
\begin{aligned}
\dot{x}(t) & =f(t, x(t), z(t)) \\
0 & =g(t, x(t), z(t))
\end{aligned}
$$

con $f: \mathbb{R} \times \mathbb{R}^{n} \times \mathbb{R}^{m} \rightarrow \mathbb{R}^{n}$ y $g: \mathbb{R} \times \mathbb{R}^{n} \times \mathbb{R}^{m} \rightarrow \mathbb{R}^{m}$ suficientemente suaves. A las variables $x$ las llamaremos diferenciales y a las variables $z$ algebraicas.

Llamamos

$$
E=\left[\begin{array}{ll}
I & 0 \\
0 & 0
\end{array}\right], \quad X=\left[\begin{array}{l}
x \\
z
\end{array}\right], \quad F=\left[\begin{array}{l}
f \\
g
\end{array}\right]
$$

con $I$ la matriz identidad de orden $n$. Entonces, matricialmente, el sistema (1.3)-(1.4) resulta,

$$
E \dot{X}(t)=F(X(t))
$$

Observar que la singularidad de $E$ transforma a (1.5) en un SDA. 
(ii) Sistemas Diferenciales Algebraicos con Control (SDAC)

Tienen la forma general:

$$
\begin{aligned}
& F(\dot{x}(t), x(t), u(t), t)=0 \\
& G(x(t), u(t), y(t), t)=0
\end{aligned}
$$

donde $x(t)$ es el vector de variables de estado del sistema; $u(t)$ es la entrada o control; $y(t)$ es la salida; y $F$ y $G$ son funciones vectoriales a valores en espacios de dimensiones apropiadas. Generalmente, (1.6) y (1.7) son llamadas ecuaciones de estado y de salida del sistema, respectivamente.

Un caso especial de (1.6)-(1.7) es

$$
\begin{aligned}
E(t) \dot{x}(t) & =H(x(t), u(t), t) \\
y(t) & =J(x(t), u(t), t)
\end{aligned}
$$

con $H$ y $J$ funciones vectoriales a valores en espacios de dimensiones adecuadas y $E(t)$ singular.

Si $H$ y $J$ son funciones lineales de $x(t)$ y $u(t)$, otra forma especial de (1.8)-(1.9) es el sistema lineal singular:

$$
\begin{aligned}
E \dot{x}(t) & =A x(t)+B u(t) \\
y(t) & =C x(t)
\end{aligned}
$$

con $x(t) \in \mathbb{R}^{n}, u(t) \in \mathbb{R}^{m}, y(t) \in \mathbb{R}^{r} ; E, A \in \mathbb{R}^{n \times n}, B \in \mathbb{R}^{n \times m}$ y $C \in \mathbb{R}^{n \times r}$ matrices constantes. Esta forma es posiblemente la más estudiada hasta el momento, [13]. 


\subsection{Aplicaciones}

Existen diferentes clases de problemas donde los SDA aparecen frecuentemente: problemas variacionales con restricciones, modelado de redes, reducción de modelo y perturbación singular (que se tratará en el Capítulo 4), discretización de ecuaciones diferenciales parciales, diferentes procesos químicos, etc.

En esta Sección, y a modo de ejemplo, describiremos brevemente como surgen los SDA en el tratamiento de problemas variacionales con restricciones. Para ello supongamos un sistema mecánico restringido con posición $x$, velocidad $u$, energía cinética $T(x, u)$, fuerza externa $f(x, u, t)$ y restricción $\phi(x)=0$. La formulación de Euler-Lagrange [14], da lugar a las ecuaciones:

$$
\begin{aligned}
\dot{x} & =u \\
\frac{d}{d t} \frac{\partial}{\partial u} T(x, u) & =\frac{\partial T}{\partial x}+f(x, u, t)+G^{T} \lambda \\
0 & =\phi(x)
\end{aligned}
$$

donde $G=\frac{\partial \phi}{\partial x}, G^{T}$ es la traspuesta de la matriz $G$, y $\lambda$ es el multiplicador de Lagrange. Este sistema puede reescribirse como

$$
\begin{aligned}
\frac{\partial^{2} T}{\partial u^{2}} \dot{u} & =g(x, u, t)+G^{T} \lambda \\
\dot{x} & =u \\
0 & =\phi(x),
\end{aligned}
$$

que resulta un SDA en las variables $u, x$ y $\lambda$. Si $\frac{\partial^{2} T}{\partial u^{2}}$ es una matriz definida positiva, entonces, premultiplicando (1.15) por $\left(\frac{\partial^{2} T}{\partial u^{2}}\right)^{-1}$, el sistema (1.15)(1.17) se transforma en un SDAS. 
Las ecuaciones de movimiento de un péndulo de longitud $L$ constituyen un ejemplo clásico de un problema variacional que da lugar a un SDA. Si $g$ es la constante gravitacional, $\lambda$ la fuerza en la barra, $\mathrm{y}(x, y)$ las coordenadas cartesianas de la bola infinitesimal de masa uno, se obtiene el SDA,

$$
\begin{aligned}
\dot{x} & =\lambda x \\
\ddot{x} & =\lambda y-g \\
0 & =x^{2}+y^{2}-L^{2}
\end{aligned}
$$

Diferentes problemas en robótica han sido formulados como SDA utilizando este enfoque variacional, [15]. Un ejemplo es un brazo de robot que se mueve con un punto final en contacto con una superficie. El movimiento de este objeto puede modelarse como,

$$
\begin{aligned}
M(x) \ddot{x}+G(x, \dot{x}) & =u+B^{T}(x) \lambda \\
0 & =\phi(x),
\end{aligned}
$$

con $B=\phi_{x}, x \in \mathbb{R}^{n}, \lambda \in \mathbb{R}^{m}, u \in \mathbb{R}^{n} . M$ es la matriz de masa, $G$ caracteriza los efectos de Coriolis, centrífugo y gravitacional del sistema, $u$ es la entrada dada por el torque aplicado a las juntas, $\phi$ representa la superficie de contacto, y $B^{T} \lambda$ es el vector que representa la fuerza de contacto. Este sistema puede transformarse, mediante la sustitución $\dot{x}=v$, en un SDA.

Por último, mencionaremos otro ejemplo de SDA que surge a partir de un problema variacional con restricciones: control óptimo con controles sin restricción. En este tipo de problemas existe un proceso dado por

$$
\dot{x}=f(x, u, t)
$$


y un costo

$$
J[x, u]=\int_{t_{0}}^{t_{1}} g(x, u, s) d s .
$$

El objetivo es elegir el control $u$ para lograr minimizar el costo (1.24) sujeto a (1.23) y a ciertas condiciones iniciales y de borde. Las ecuaciones variacionales de (1.23)-(1.24) para el problema de tiempo fijo y punto final fijo dan lugar al SDAS:

$$
\begin{aligned}
\dot{x} & =f(x, u, t) \\
\dot{\lambda} & =-g_{x}(x, u, t)-f_{x}^{T} \lambda \\
0 & =g_{u}(x, u, t)+f_{u}^{T} \lambda .
\end{aligned}
$$

\subsection{Esquema general}

En este capítulo, hemos introducido de modo general el concepto de SDA, y hemos comentado dos enfoques diferentes: numérico y geométrico. Hemos citado, además, algunas clases particulares de SDA; y también algunas problemas de aplicación donde los SDA surgen naturalmente.

En el Capítulo 2 estableceremos que tipo de hipótesis se pedirán para los campos vectoriales y funciones con los que se trabajará a lo largo de la tesis. Se enunciarán además algunos resultados standard cuya validez se garantizará a partir de las hipótesis que mencionamos antes.

En el Capítulo 3 nos ocuparemos especialmente de los SDAS, analizando bajo qué condiciones existen y son únicas las soluciones (caso regular). En particular, hallaremos el espacio de configuración de un SDAS, también conocido como dominio de condiciones iniciales consistentes.

En los Capítulos 4 y 5 aplicaremos los conceptos fundamentales detallados en el Capítulo 3 a dos problemas particulares. En el Capítulo 4 trabaja- 
remos con Sistemas de Perturbación Singular. Estos sistemas (que dependen de uno o varios parámetros de perturbación) se convierten en SDA al anular los parámetros de perturbación. Luego, es posible aproximar sus trayectorias a partir de las trayectorias del SDA asociado. En particular, se obtendrá un resultado de aproximación cuando el SDA que resulta es de índice 2. En el Capítulo 5 trabajaremos con sistemas no-lineales con control, sujetos a restricciones algebraicas. Entonces, considerando a las variables de control como variables algebraicas, hallaremos la variedad maximal invariante por realimentación de estados correspondiente al sistema no-lineal. Es decir, construiremos un control por realimentación y hallaremos una subvariedad $\mu$ de aquella que definen las restricciones algebraicas, de modo tal que, las trayectorias del sistema realimentado, con condiciones iniciales en $\mu$, evolucionan sobre $\mu$. Además, se aplicará el algoritmo propuesto en este capítulo para hallar la dinámica cero de un sistema no-lineal con múltiples entradas y salidas.

Por último, en el Capítulo 6 enunciaremos las conclusiones, resumiendo las contribuciones de la tesis y citando algunas futuras líneas de investigación. 


\section{Capítulo 2}

\section{Preliminares}

A lo largo de la tesis trabajaremos con campos vectoriales y funciones suficientemente diferenciables (en general $C^{\infty}$ ), que estarán definidos sobre conjuntos abiertos de espacios euclídeos ó sobre variedades, que precisaremos en cada caso.

El objetivo de trabajar con campos ó funciones suficientemente diferenciables es garantizar la validez de algunos resultados, en particular el Teorema de la Función Implícita (TFI) y el Teorema de Existencia y Unicidad de Solución para Ecuaciones Diferenciales Ordinarias, que enunciaremos a continuación, [16].

\section{Teorema de la Función Implícita:}

Sean $U \subset E, V \subset F$ abiertos de espacios de Banach $E$ y $F$, y

$$
f: U \times V \rightarrow G
$$

de clase $C^{r}, r \geq 1$. Para algún $x_{0} \in U, y_{0} \in V$, supongamos que

$$
D_{2} f\left(x_{0}, y_{0}\right): F \rightarrow G
$$

es isomorfismo. Entonces existen entornos $U_{0}$ de $x_{0}$ y $W_{0}$ de $f\left(x_{0}, y_{0}\right)$ y una 
única aplicación

$$
g: U_{0} \times W_{0} \rightarrow V
$$

tal que para todo $(x, w) \in U_{0} \times W_{0}, f(x, g(x, w))=w$.

\section{Teorema de Existencia y Unicidad de Solución para Ecuaciones Diferenciales Ordinarias:}

Sea $E$ un espacio de Banach y $X: U \subset E \rightarrow E$ de clase $C^{\infty}$. Para cada $x_{0} \in U$, existe una curva $c: I \rightarrow U$ en $x_{0}$ tal que $c^{\prime}(t)=X(c(t)), \forall t \in I$. Dos cualesquiera de tales curvas son iguales sobre la intersección de sus dominios. Además, existe un entorno $U_{0}$ de $x_{0} \in U$, un número real $a>0$ y una aplicación $C^{\infty} F: U_{0} \times I \rightarrow E$, donde $I=(-a, a)$, tal que la curva $c_{u}(t): I \rightarrow E$, definida por $c_{u}(t)=F(t, u)$ es una curva en $u \in E$ que satisface la ecuación diferencial $c_{u}^{\prime}(t)=X\left(c_{u}(t)\right), \forall t \in I$.

Finalmente observamos que todos los resultados que se obtendrán serán locales, es decir serán válidos en un entorno del punto de operación donde se trabaje en cada caso. 


\section{Capítulo 3}

\section{SDA Semiexplícitos}

En este capítulo analizaremos algunos aspectos vinculados a una clase particular de Sistemas Diferenciales Algebraicos: los Sistemas Diferenciales Algebraicos Semiexplícitos (SDAS), ya mencionados en la Sección 1.3. Trabajaremos con SDAS autónomos de la forma

$$
\begin{aligned}
\dot{x}(t) & =f(x(t), z(t)) \\
0 & =g(x(t), z(t))
\end{aligned}
$$

con $f$ y $g$ definidos sobre $D_{1} \times D_{2}$, con $D_{1} \subset \mathbb{R}^{n}$ y $D_{2} \subset \mathbb{R}^{m}$ abiertos; suponemos además que $g$ toma valores en $\mathbb{R}^{m}$. Nos ocuparemos esencialmente de los SDAS desde el punto de vista geométrico. Existen en la literatura diferentes trabajos que analizan a los SDA con un enfoque geométrico, como por ejemplo [10], [17], [18], [19] y [20]. La idea básica con que se trabaja en estos artículos es la de asociar al SDA un campo vectorial definido sobre una cierta variedad, de modo tal que las soluciones del SDA y las del campo vectorial estén en correspondencia uno a uno. En [17] y [18] se estudia la existencia y unicidad de solución para SDA más generales; se introducen además algunos conceptos tales como regularidad e índice de un 
SDA, campo vectorial y espacio de configuración correspondientes a un SDA, familia de variedades restringidas, etc. En [21] hemos aplicado estas ideas al caso particular de los SDAS de la forma (3.1)-(3.2), y hemos construido un algoritmo que permite describir en forma explícita la familia de variedades restringidas, campo vectorial y espacio de configuración asociados al sistema, y que detallaremos en la Sección 3.2. Luego, aplicaremos este algoritmo al caso particular de un SDO con restricciones algebraicas. Además, analizando dos ejemplos simples, ilustraremos algunos de los conceptos y resultados presentados en el capítulo.

\subsection{Regularidad de SDAS}

Comenzaremos esta Sección dando la definición de solución correspondiente a un SDA y a un campo vectorial, respectivamente.

Definición 3.1: Una solución del sistema (3.1)-(3.2) es una aplicación diferenciable $c=\left(c_{1}, c_{2}\right): I \rightarrow \mathbb{R}^{n+m}$ (donde $I$ representa un intervalo real) tal que, para todo $t \in I$,

$$
\begin{aligned}
\frac{d c_{1}}{d t}(t) & =f\left(c_{1}(t), c_{2}(t)\right) \\
0 & =g\left(c_{1}(t), c_{2}(t)\right) .
\end{aligned}
$$

Dada $M$ variedad diferenciable, llamamos $T M$ al fibrado tangente de $M$ y $T_{x} M$ al espacio tangente a $M$ en $x$.

Definición 3.2: Un campo vectorial sobre $M$ es una aplicación $v: M \rightarrow T M$ tal que $v(x) \in T_{x} M$, para todo $x \in M$. Una solución del campo vectorial $v$ por $x_{0} \in M$ es una aplicación diferenciable $w: I \rightarrow M$ ( $I$ intervalo real) que 
pasa por $x_{0}$, tal que, para todo $t \in I$,

$$
\frac{d w}{d t}(t)=v(w(t))
$$

Rheinboldt, [10], fue el primer autor en dar una caracterización geométricodiferencial para cierta clase de SDA autónomos. Esta caracterización dio lugar a considerar a un SDA como una descripción implícita de un campo vectorial, y a introducir la noción de SDA regular.

Definición 3.3: el SDAS (3.1)-(3.2) se dice regular si existe una variedad diferenciable $M \subset \mathbb{R}^{n+m}$ y un campo vectorial $v: M \rightarrow T M$ tal que una aplicación diferenciable $w: I \rightarrow M$ es una solución del campo vectorial sii la aplicación $c=j \circ w: I \rightarrow \mathbb{R}^{n+m}$ es una solución de (3.1)-(3.2); siendo $j: M \rightarrow \mathbb{R}^{n+m}$ la inclusión canónica.

A la variedad $M$ maximal que cumple con la definición anterior se la llama espacio de configuración del SDAS (3.1)-(3.2) y al campo vectorial $v$ campo vectorial correspondiente al sistema (3.1)-(3.2).

Observación 3.1: cuando la regularidad de un SDAS (o en general de un SDA) ha sido probada, pueden obtenerse resultados de existencia y unicidad de soluciones para ese sistema empleando la teoría de existencia y unicidad de soluciones ya desarrollada para campos vectoriales.

En [17] y [18] se caracterizaron el espacio de configuración y el campo vectorial correspondiente para cierta clase de SDA. Se estableció, además, una condición suficiente que garantiza regularidad para un SDA dado. Resumiremos esta caracterización para el caso particular de los SDAS. 
Definición 3.4: El conjunto correspondiente al sistema (3.1)-(3.2) es

$$
N=\left\{(x, z, \dot{x}, \dot{z}) \in \mathbb{R}^{2(n+m)}: \dot{x}=f(x, z), 0=g(x, z)\right\}
$$

Observación 3.2: Una aplicación $c=\left(c_{1}, c_{2}\right): I \rightarrow \mathbb{R}^{n+m}$ es solución del SDAS (3.1)-(3.2) sii $\left(c(t), \frac{d c}{d t}(t)\right) \in N$, para todo $t \in I$.

Si llamamos

$$
M_{1}=\pi_{1}(N)
$$

siendo $\pi_{1}: \mathbb{R}^{2(n+m)} \rightarrow \mathbb{R}^{n+m}$ la proyección canónica sobre las primeras $n+m$ componentes, se tiene que: si $M_{1}$ es variedad diferenciable, entonces $c=\left(c_{1}, c_{2}\right)$ es solución del SDAS (3.1)-(3.2) sii $\left(c(t), \frac{d c}{d t}(t)\right) \in N \cap T M_{1}$, para todo $t \in I$.

Definiendo ahora,

$$
M_{2}=\pi_{1}\left(N \cap T M_{1}\right)
$$

y suponiendo a $M_{2}$ variedad diferenciable, se tiene nuevamente que $c=\left(c_{1}, c_{2}\right)$ es solución de (3.1)-(3.2) sii $\left(c(t), \frac{d c}{d t}(t)\right) \in N \cap T M_{2}$, para todo $t \in I$.

Este proceso puede continuarse hasta que

$$
M_{i}=\pi_{1}\left(N \cap T M_{i-1}\right)
$$

sea variedad diferenciable, y se detiene si $M_{i+1}=M_{i}$.

Definición 3.5: Sea $N$ el conjunto correspondiente asociado al sistema (3.1)-(3.2). Se define la familia de variedades $\left\{M_{i}\right\}_{i=0}^{s}$, recursivamente en la forma:

(i) $M_{0}=\mathbb{R}^{n+m}$

(ii) $M_{i+1}=\pi_{1}\left(N \cap T M_{i}\right)$, para $i=0,1, \ldots, s-1$, 
siendo $s$ el mayor entero tal que $M_{i}$ es variedad diferenciable y $M_{s-1} \neq M_{s}$. A la familia $\left\{M_{i}\right\}_{i=0}^{s}$ se la llama familia de variedades restringidas y al entero $s$ grado del SDA.

Observar que $\operatorname{dim} M_{i+1} \leq \operatorname{dim} M_{i}$, para todo $i=0,1, \ldots, s-1$. Si $\operatorname{dim}\left(M_{i+1}\right)=$ $\operatorname{dim}\left(M_{i}\right)>0$, se tiene

$$
T M_{i}=T M_{i+1} \cup\left(\bigcup_{x \in M_{i}-M_{i+1}} T_{x} M_{i}\right)
$$

y en consecuencia

$$
N \cap T M_{i}=N \cap T M_{i+1}
$$

luego, $M_{i+1}=M_{i+2}$. Se concluye entonces que el grado $s$ del SDA satisface $s \leq n+m$.

El siguiente resultado garantiza regularidad para el sistema (3.1)-(3.2):

Proposición 3.1: Sea $N$ el conjunto correspondiente al sistema (3.1)-(3.2), y sea $\left\{M_{i}\right\}_{i=0}^{s}$ la familia de variedades restringidas asociada a este sistema, que suponemos de grado $s$. Si para cada $(x, z) \in M_{s}$ existe un único $(\dot{x}, \dot{z}) \in$ $T_{(x, z)} M_{s}$ tal que $(x, z, \dot{x}, \dot{z}) \in N$, entonces (3.1)-(3.2) es un SDA regular.

Definición 3.6: Si (3.1)-(3.2) es un SDA regular, al número $s$ se lo llama indice del sistema.

Observación 3.3: Es importante resaltar que dado un SDA regular, existe un único campo vectorial que lo representa. Además, la variedad $M=M_{s}$ obtenida con el procedimiento anterior es el espacio de configuración del sistema. Esta variedad constituye el conjunto de condiciones iniciales consistentes del SDA. Es decir, si tomamos una condición inicial sobre $M$, la 
solución del SDA con esta condición inicial, evoluciona sobre la variedad a lo largo del tiempo.

En [21], suponiendo regularidad para (3.1)-(3.2), obtuvimos una descripción explícita de la familia $\left\{M_{i}\right\}_{i}$, para poder establecer, también explícitamente, el espacio de configuración y el campo vectorial asociados a este sistema. A continuación se detallará esta descripción para los casos particulares de SDAS de índices 1, 2 y 3, para luego generalizarlo al caso de índice $s$.

\subsection{Campo vectorial y espacio de configuración}

En esta sección se establecerá un algoritmo para caracterizar la familia de variedades restringidas ya definida antes, y se obtendrán, además, algunos resultados que garantizan índices 1, 2, 3 y en general índice $s$ para un SDAS del tipo (3.1)-(3.2). Estos resultados, además de algunos comentarios, se intercalarán en los distintos pasos del algoritmo, de modo tal de poder continuar con el paso siguiente de manera más simple. Las hipótesis que se pedirán a lo largo del algoritmo son de naturaleza local, es decir, valdrán en un entorno de algún punto de operación (aunque no lo aclaremos en cada caso). Entonces, los campos vectoriales quedarán definidos también localmente.

- Paso 0:

$M_{0}=\mathbb{R}^{n+m}$

- Paso 1:

$M_{1}=\pi_{1}(N)=g^{-1}(0)$ 
- Paso 2:

$$
M_{2}=\pi_{1}\left(N \cap T M_{1}\right)
$$

Para determinar la forma explícita de $M_{2}$ observar que $(x, z, \dot{x}, \dot{z}) \in N \cap T M_{1}$ sii

$$
\left\{\begin{aligned}
\dot{x} & =f(x, z) \\
0 & =g(x, z) \\
0 & =\frac{\partial g}{\partial x} \dot{x}+\frac{\partial g}{\partial z} \dot{z}
\end{aligned}\right.
$$

Caso I:

$$
r g\left(\frac{\partial g}{\partial z}\right)=m \text {. }
$$

Proposición 3.2: Si se cumple (3.3), el sistema (3.1)-(3.2) tiene índice 1.

Demostración:

Por hipótesis, la matriz $\left(\frac{\partial g}{\partial z}\right)$ tiene inversa, entonces es posible obtener explícitamente una expresión para $\dot{z}$,

$$
\dot{z}=-\left(\frac{\partial g}{\partial z}\right)^{-1} \frac{\partial g}{\partial x} f(x, z)
$$

y se tiene que $M_{2}=M_{1}$; y entonces, el sistema (3.1)-(3.2) tiene índice 1 .

Observación 3.3: Si el SDAS (3.1)-(3.2) tiene índice 1, el espacio de configuración es $M_{1}$, y el campo vectorial correspondiente es

$$
v_{1}: M_{1} \rightarrow T M_{1}, \text { con } v_{1}(x, z)=\left(x, z, f(x, z),-\left(\frac{\partial g}{\partial z}\right)^{-1} \frac{\partial g}{\partial x} f(x, z)\right) . \square
$$

Nuevamente observemos que si bien consideramos a $M_{1}$ como dominio de $v_{1}$, en realidad este dominio estará restringido al conjunto de puntos de $M_{1}$ donde se cumple la hipótesis (3.3). De la misma forma, los dominios de los campos vectoriales que se irán definiendo en adelante estarán restringidos al conjunto de puntos que verifiquen las hipótesis de rango constante que se pedirán en cada caso. 
Entonces, si el sistema tiene índice 1, el algoritmo termina. Si en cambio,

\section{Caso II:}

$$
r g\left(\frac{\partial g}{\partial z}\right)=m_{1}<m
$$

entonces, existe una permutación $P$ tal que $z_{1}=P z$ y $g_{1}=P g$, con

$$
z_{1}=\left[\begin{array}{l}
z_{11} \\
z_{12}
\end{array}\right], g_{1}=\left[\begin{array}{l}
g_{11} \\
g_{12}
\end{array}\right]
$$

donde $z_{11} \in \mathbb{R}^{m_{1}}, z_{12} \in \mathbb{R}^{m-m_{1}}, g_{11}: \mathbb{R}^{n+m} \rightarrow \mathbb{R}^{m_{1}}, g_{12}: \mathbb{R}^{n+m} \rightarrow \mathbb{R}^{m-m_{1}}$; y de modo tal que

$$
r g\left(\frac{\partial g_{11}}{\partial z_{11}}\right)=m_{1}
$$

Luego, la aplicación

$$
\left(x, w_{11}, z_{12}\right)=\phi_{1}\left(x, z_{11}, z_{12}\right)=\left(x, g_{11}\left(x, z_{11}, z_{12}\right), z_{12}\right)
$$

resulta un difeomorfismo. Además, aplicando a $g_{11}\left(x, z_{11}, z_{12}\right)$ el TFI (ver Capítulo 2), existe una función suave $\alpha_{1}\left(x, w_{11}, z_{12}\right)$ tal que

$$
g_{11}\left(x, \alpha_{1}\left(x, w_{11}, z_{12}\right), z_{12}\right)=w_{11}
$$

y se verifica

$$
g_{11} \circ \phi_{1}^{-1}\left(x, w_{11}, z_{12}\right)=w_{11} .
$$

Entonces, mediante el cambio de coordenadas definido por $\phi_{1}$, la restricción algebraica $0=g_{1}\left(x, z_{11}, z_{12}\right)$ es equivalente a:

$$
\begin{aligned}
w_{11} & =0 \\
g_{12} \circ \phi_{1}^{-1}\left(x, w_{11}, z_{12}\right) & =0
\end{aligned}
$$

Sin embargo, bajo la hipótesis (3.4), puede probarse que: 
Proposición 3.3: Existe una función suave $H_{1}$ que depende sólo de $x$ y a valores en $\mathbb{R}^{m-m_{1}}$, tal que (3.5)-(3.6) es equivalente a

$$
\begin{aligned}
w_{11} & =0 \\
H_{1}(x) & =0
\end{aligned}
$$

Demostración:

Si llamamos $\bar{g}_{12}\left(x, w_{11}, z_{12}\right)=g_{12} \circ \phi_{1}^{-1}\left(x, w_{11}, z_{12}\right)$, y $w_{11}=\left(w_{11}^{1}, \ldots, w_{11}^{m_{1}}\right)$, $z_{12}=\left(z_{12}^{1}, \ldots, z_{12}^{m-m_{1}}\right)$, entonces la matriz Jacobiana $\frac{\partial\left(g_{1} \circ \phi_{1}^{-1}\right)}{\partial\left(w_{11}, z_{12}\right)}$ en cada punto $\left(x, w_{11}, z_{12}\right)$ es:

$$
\left[\begin{array}{ccccccc}
1 & 0 & \ldots & 0 & 0 & \cdots & 0 \\
0 & 1 & \ldots & 0 & 0 & \cdots & 0 \\
\vdots & \vdots & & \vdots & \vdots & & \vdots \\
0 & 0 & \ldots & 1 & 0 & \ldots & 0 \\
\frac{\partial \bar{g}_{12}^{1}}{\partial w_{11}^{1}} & \frac{\partial \bar{g}_{12}^{1}}{\partial w_{11}^{2}} & \cdots & \frac{\partial \bar{g}_{12}^{1}}{\partial w_{11}^{m_{1}}} & \frac{\partial \bar{g}_{12}^{1}}{\partial z_{12}^{1}} & \cdots & \frac{\partial \bar{g}_{12}^{1}}{\partial z_{12}^{m-m_{1}}} \\
& & & & & & \\
\frac{\partial \bar{g}_{12}^{m-m_{1}}}{\partial w_{11}^{1}} & \frac{\partial \bar{g}_{12}^{m-m_{1}}}{\partial w_{11}^{2}} & \ldots & \frac{\partial \bar{g}_{12}^{m-m_{1}}}{\partial w_{11}^{m}} & \frac{\partial \bar{g}_{12}^{m-m_{1}}}{\partial z_{12}^{1}} & \cdots & \frac{\partial \bar{g}_{12}^{m-m_{1}}}{\partial z_{12}^{m-m_{1}}}
\end{array}\right]
$$

donde $\frac{\partial \bar{g}_{12}^{i}}{\partial w_{11}^{l}}, \frac{\partial \bar{g}_{12}^{i}}{\partial z_{12}^{j}}$, con $1 \leq i, j \leq m-m_{1}, 1 \leq l \leq m_{1}$, están evaluados en $\left(x, w_{11}, z_{12}\right)$. Además, como

$$
r g\left(\frac{\partial g_{1}}{\partial\left(z_{11}, z_{12}\right)}\right)=m_{1}
$$

entonces

$$
r g\left(\frac{\partial\left(g_{1} \circ \phi_{1}^{-1}\right)}{\partial\left(w_{11}, z_{12}\right)}\right)=m_{1}
$$

de donde se concluye que

$$
\frac{\partial \bar{g}_{12}^{i}}{\partial z_{12}^{j}}\left(x, w_{11}, z_{12}\right)=0
$$


para todo $1 \leq i, j \leq m-m_{1}$. Se tiene entonces que $\bar{g}_{12}$ no depende de $z_{12}$. Es decir, $\bar{g}_{12}\left(x, w_{11}, z_{12}\right)=\tilde{g}_{12}\left(x, w_{11}\right)$.

Luego (3.5)-(3.6) es equivalente a

$$
\begin{aligned}
w_{11} & =0 \\
H_{1}(x) & =0
\end{aligned}
$$

con $H_{1}(x)=\tilde{g}_{12}(x, 0)$.

Para determinar en este caso la forma explícita de $M_{2}$, trabajando por simplicidad en coordenadas $\left(x, w_{11}, z_{12}\right)$, se tiene que $\left(x, w_{11}, z_{12}, \dot{x}, \dot{w}_{11}, \dot{z}_{12}\right) \in$ $H \cap T M_{1}$ sii:

$$
\left\{\begin{aligned}
\dot{x} & =f_{1}\left(x, w_{11}, z_{12}\right) \\
0 & =w_{11} \\
0 & =H_{1}(x) \\
0 & =\dot{w}_{11} \\
0 & =\frac{\partial H_{1}}{\partial x} f_{1}\left(x, w_{11}, z_{12}\right)
\end{aligned}\right.
$$

con $f_{1}\left(x, w_{11}, z_{12}\right)=f\left(x, \alpha_{1}\left(x, w_{11}, z_{12}\right), z_{12}\right)$. Luego,

$$
M_{2}=\left\{\left(x, w_{11}, z_{12}\right): w_{11}=0, H_{1}(x)=0, L_{f_{1}} H_{1}\left(x, w_{11}, z_{12}\right)=0\right\},
$$

donde $L_{f_{1}} H_{1}\left(x, w_{11}, z_{12}\right)=\frac{\partial H_{1}}{\partial x} f_{1}\left(x, w_{11}, z_{12}\right)$.

Volvemos ahora a nuestro algoritmo,

- Paso 3:

$$
M_{3}=\pi_{1}\left(N \cap T M_{2}\right)
$$

Razonando como antes se tendrá que $\left(x, w_{11}, z_{12}, \dot{x}, \dot{w}_{11}, \dot{z}_{12}\right) \in N \cap T M_{2}$ sii 


$$
\left\{\begin{aligned}
\dot{x} & =f_{1}\left(x, w_{11}, z_{12}\right) \\
0 & =w_{11} \\
0 & =H_{1}(x) \\
0 & =\dot{w}_{11} \\
0 & =L_{f_{1}} H_{1}\left(x, w_{11}, z_{12}\right) \\
0 & =\frac{\partial\left(L_{f_{1}} H_{1}\right)}{\partial x} f_{1}\left(x, w_{11}, z_{12}\right)+\frac{\partial\left(L_{f_{1}} H_{1}\right)}{\partial z_{12}} \dot{z}_{12}
\end{aligned}\right.
$$

Caso I:

$$
r g\left(\frac{\partial\left(L_{f_{1}} H_{1}\right)}{\partial z_{12}}\right)=m-m_{1}
$$

Proposición 3.4: Si suponemos (3.9), entonces el sistema (3.1)-(3.2) tiene índice 2.

Demostración:

Por hipótesis (3.9), se puede obtener una expresión para $\dot{z}_{12}$ :

$$
\dot{z}_{12}=-\left(\frac{\partial\left(L_{f_{1}} H_{1}\right)}{\partial z_{12}}\right)^{-1}\left(\frac{\partial\left(L_{f_{1}} H_{1}\right)}{\partial x}\right) f_{1}\left(x, w_{11}, z_{12}\right),
$$

y se concluye entonces que $M_{3}=M_{2}$.

Observación 3.4: si el SDAS (3.1)-(3.2) tiene índice 2 el espacio de configuración es $M_{2}$, y el campo vectorial correspondiente resulta

$$
\begin{gathered}
v_{2}: M_{2} \rightarrow T M_{2}, \operatorname{con} v_{2}\left(x, w_{11}, z_{12}\right)= \\
\left(x, w_{11}, z_{12}, f_{1}\left(x, w_{11}, z_{12}\right), 0,-\left(\frac{\partial\left(L_{f_{1}} H_{1}\right)}{\partial z_{12}}\right)^{-1} \frac{\partial\left(L_{f_{1}} H_{1}\right)}{\partial x} f_{1}\left(x, w_{11}, z_{12}\right)\right) .
\end{gathered}
$$

En este caso el algoritmo termina.

Caso II:

$$
r g\left(\frac{\partial\left(L_{f_{1}} H_{1}\right)}{\partial z_{12}}\right)=m_{2}<m-m_{1} .
$$

Nuevamente, podemos reordenar las componentes de $z_{12}$ y $L_{f_{1}} H_{1}$, y llamando $z_{2}=z_{12}, g_{2}=L_{f_{1}} H_{1}$, se tendrá 


$$
z_{2}=\left[\begin{array}{l}
z_{21} \\
z_{22}
\end{array}\right], g_{2}=\left[\begin{array}{l}
g_{21} \\
g_{22}
\end{array}\right]
$$

con $z_{21} \in \mathbb{R}^{m_{2}}, z_{22} \in \mathbb{R}^{m-\left(m_{1}+m_{2}\right)}, g_{21}: \mathbb{R}^{m-m_{1}} \rightarrow \mathbb{R}^{m_{2}}, g_{22}: \mathbb{R}^{m-m_{1}} \rightarrow \mathbb{R}^{m-\left(m_{1}+m_{2}\right)}$, de modo tal que

$$
r g\left(\frac{\partial g_{21}}{\partial z_{21}}\right)=m_{2}
$$

Luego, la aplicación

$$
\left(x, w_{11}, w_{21}, z_{22}\right)=\phi_{2}\left(x, w_{11}, z_{21}, z_{22}\right)=\left(x, w_{11}, g_{21}\left(x, w_{11}, z_{21}, z_{22}\right), z_{22}\right)
$$

es un difeomorfismo; y aplicando a $g_{21}\left(x, w_{11}, z_{21}, z_{22}\right)$ el TFI, existe una función suave $\alpha_{2}\left(x, w_{11}, w_{21}, z_{22}\right)$ tal que,

$$
g_{21}\left(x, w_{11}, \alpha_{2}\left(x, w_{11}, w_{21}\right), z_{22}\right)=w_{21}
$$

Además, se verifica:

$$
g_{21} \circ \phi_{2}^{-1}\left(x, w_{11}, w_{21}, z_{22}\right)=w_{21}
$$

Entonces, bajo el cambio de coordenadas definido por $\phi_{2}$, la restricción algebraica $0=g_{2}\left(x, w_{11}, z_{21}, z_{22}\right)$ es equivalente a:

$$
\begin{aligned}
w_{21} & =0 \\
g_{22} \circ \phi_{2}^{-1}\left(x, w_{11}, w_{21}, z_{22}\right) & =0
\end{aligned}
$$

De la misma manera que en la Proposición 3.3, y suponiendo $r g\left(\frac{\partial g_{21}}{\partial z_{21}}\right)=m_{2}$, se puede probar que existe $H_{2}(x)$ tal que, (3.11)-(3.12) es equivalente a:

$$
\begin{aligned}
w_{21} & =0 \\
H_{2}(x) & =0
\end{aligned}
$$


y entonces $\left(x, w_{11}, w_{21}, z_{22}, \dot{x}, \dot{w}_{11}, \dot{w}_{21}, \dot{z}_{22}\right) \in N \cap T M_{2}$ sii

$$
\left\{\begin{aligned}
\dot{x} & =f_{2}\left(x, w_{11}, w_{21}, z_{22}\right) \\
0 & =w_{11} \\
0 & =H_{1}(x) \\
0 & =\dot{w}_{11} \\
0 & =w_{21} \\
0 & =H_{2}(x) \\
0 & =\dot{w}_{21} \\
0 & =L_{f_{2}} H_{2}\left(x, w_{11}, w_{21}, z_{22}\right)
\end{aligned}\right.
$$

donde $f_{2}\left(x, w_{11}, w_{21}, z_{22}\right)=f_{1}\left(x, w_{11}, \alpha_{2}\left(x, w_{11}, w_{21}, z_{22}\right), z_{22}\right)$.

La forma explícita de $M_{3}$ es,

$$
\begin{gathered}
M_{3}=\left\{\left(x, w_{11}, w_{21}, z_{22}\right): w_{11}=0, H_{1}(x)=0, w_{21}=0, H_{2}(x)=0,\right. \\
\left.L_{f_{2}} H_{2}\left(x, w_{11}, w_{21}, z_{22}\right)=0\right\} .
\end{gathered}
$$

- Paso 4:

$$
M_{4}=\pi_{1}\left(N \cap T M_{3}\right)
$$

\section{Caso I:}

$$
r g\left(\frac{\partial\left(L_{f_{2}} H_{2}\right)}{\partial z_{22}}\right)=m-\left(m_{1}+m_{2}\right) .
$$

Proposición 3.5: Si se verifica (3.13), entonces el sistema (3.1)-(3.2) tiene índice 3.

Observación 3.5: Si (3.1)-(3.2) tiene índice 3, el espacio de configuración es $M_{3}$ y el campo vectorial correspondiente

$$
v_{3}: M_{3} \rightarrow T M_{3}
$$

con

$$
v_{3}\left(x, w_{11}, w_{12}, z_{22}\right)=
$$

$\left.\left(x, w_{11}, w_{21}, z_{22}, f_{2}\left(x, w_{11}, w_{21}, z_{22}\right), 0,0,-\left(\frac{\partial\left(L_{f_{2}} H_{2}\right)}{\partial z_{22}}\right)^{-1} \frac{\partial\left(L_{f_{2}} H_{2}\right)}{\partial x} f_{2}\left(x, w_{11}, w_{21}, z_{22}\right)\right)\right)$. 


\section{Caso II:}

$$
r g\left(\frac{\partial\left(L_{f_{2}} H_{2}\right)}{\partial z_{22}}\right)=m_{3}<m-\left(m_{1}+m_{2}\right)
$$

en este caso el algoritmo continúa.

- Paso s:

$$
M_{s}=\pi_{1}\left(N \cap T M_{s-1}\right)
$$

\section{Caso I:}

$$
r g\left(\frac{\partial L_{f_{s-2}} H_{s-2}}{\partial z_{(s-2) 2}}\right)=m-\left(m_{1}+m_{2}+\ldots+m_{s-2}\right)
$$

y entonces el sistema (3.1)-(3.2) tiene índice $s-1$. Si en cambio,

\section{Caso II:}

$$
r g\left(\frac{\partial L_{f_{s-2}} H_{s-2}}{\partial z_{(s-2) 2}}\right)=m_{s-1}<m-\left(m_{1}+m_{2}+\ldots+m_{s-2}\right)
$$

como antes, renombramos $z_{s-1}=z_{(s-2) 2}, g_{s-1}=L_{f_{s-2}} H_{s-2}$, y obtenemos

$$
z_{s-1}=\left[\begin{array}{l}
z_{(s-1) 1} \\
z_{(s-1) 2}
\end{array}\right] ; g_{s-1}=\left[\begin{array}{l}
g_{(s-1) 1} \\
g_{(s-1) 2}
\end{array}\right]
$$

con

$$
\begin{gathered}
z_{(s-1) 1} \in \mathbb{R}^{m_{s-1}}, z_{(s-1) 2} \in \mathbb{R}^{m-\left(m_{1}+\ldots+m_{s-1}\right)}, \\
g_{(s-1) 1}: \mathbb{R}^{m-\left(m_{1}+\ldots+m_{s-2}\right)} \rightarrow \mathbb{R}^{m_{s-1}}, \\
g_{(s-1) 2}: \mathbb{R}^{m-\left(m_{1}+\ldots+m_{s-2}\right)} \rightarrow \mathbb{R}^{m-\left(m_{1}+\ldots+m_{s-1}\right)},
\end{gathered}
$$

tales que

$$
r g\left(\frac{\partial g_{(s-1) 1}}{\partial z_{(s-1) 1}}\right)=m_{s-1}
$$


En este caso, la aplicación

$\left(x, w_{11}, w_{21}, \ldots, w_{(s-2) 1}, w_{(s-1) 1}, z_{(s-1) 2}\right)=\phi_{s}\left(x, w_{11}, w_{21}, \ldots, w_{(s-2) 1}, z_{(s-1) 1}, z_{(s-1) 2}\right)$

es un difeomorfismo; y por el TFI existe

$$
\alpha_{s-1}\left(x, w_{11}, w_{21}, \ldots, w_{(s-2) 1}, w_{(s-1) 1}, z_{(s-1) 2}\right)
$$

función suave, tal que

$g_{(s-1) 1}\left(x, w_{11}, \ldots, w_{(s-2) 1}, \alpha_{s-1}\left(x, w_{11}, \ldots, w_{(s-2) 1}, w_{(s-1) 1}, z_{(s-1) 2}\right), z_{(s-1) 2}\right)=w_{(s-1) 1}$,

$\mathrm{y}$

$$
g_{(s-1) 1} \circ \phi_{s-1}^{-1}\left(x, w_{11}, \ldots, w_{(s-1) 1}, z_{(s-1) 2}\right)=w_{(s-1) 1}
$$

Además, existe $H_{s-1}(x)$ tal que,

$$
\begin{aligned}
w_{(s-1) 1} & =0 \\
g_{(s-1) 2} \circ \phi_{s-1}^{-1}\left(x, w_{11}, \ldots, w_{(s-1) 1}, z_{(s-1) 2}\right) & =0
\end{aligned}
$$

es equivalente a:

$$
\begin{aligned}
w_{(s-1) 1} & =0 \\
H_{s-1}(x) & =0
\end{aligned}
$$

La forma explícita de $M_{s}$ es ahora:

$$
\begin{aligned}
M_{s} & =\left\{\left(x, w_{11}, w_{21}, \ldots, w_{(s-1) 1}, z_{(s-1) 2}\right): w_{11}=0, H_{1}(x)=0, w_{21}=0,\right. \\
H_{2}(x) & \left.=0, \ldots, w_{(s-1) 1}=0, H_{s-1}(x)=0, L_{f_{s-1}} H_{s-1}\left(x, w_{11}, \ldots, w_{(s-1) 1}, z_{(s-1) 2}\right)=0\right\}
\end{aligned}
$$

con

$$
\begin{gathered}
f_{s-1}\left(x, w_{11}, \ldots, w_{(s-1) 1}, z_{(s-1) 2}\right)= \\
f_{s-2}\left(x, w_{11}, \ldots, \alpha_{s-1}\left(x, w_{11}, \ldots, w_{(s-1) 1}, z_{(s-1) 2}\right), z_{(s-1) 2}\right)
\end{gathered}
$$


- Paso $s+1$ :

$$
M_{s+1}=\pi_{1}\left(N \cap T M_{s}\right)
$$

Supondremos que

$$
r g\left(\frac{\partial\left(L_{f_{s-1}} H_{s-1}\right)}{\partial z_{(s-1) 2}}\right)=m-\left(m_{1}+m_{2}+\ldots+m_{s-1}\right) .
$$

Se tiene entonces $M_{s+1}=M_{s}$, y luego el sistema (3.1)-(3.2) tiene índice $s$. En este caso el espacio de configuración es $M_{s}$ y el campo vectorial correspondiente $v_{s}: M_{s} \rightarrow T M_{s}$, se define así:

$$
\begin{gathered}
v_{s}\left(x, w_{11}, \ldots, w_{(s-1) 1}, z_{(s-1) 2}\right)= \\
\left(x, w_{11}, \ldots, w_{(s-1) 1}, z_{(s-1) 2}, f_{s-1}\left(x, w_{11}, \ldots, w_{(s-1) 1}, z_{(s-1) 2}\right), 0, \ldots, 0\right. \\
\left.-\left(\frac{\partial L_{f_{s-1}} H_{s-1}}{\partial z_{(s-1) 2}}\right)^{-1}\left(\frac{\partial L_{f_{s-1}} H_{s-1}}{\partial x}\right) f_{s-1}\left(x, w_{11}, \ldots, w_{(s-1) 1}, z_{(s-1) 2}\right)\right) .
\end{gathered}
$$

Para terminar esta Sección establecemos el siguiente resultado que generaliza las proposiciones probadas a lo largo del algoritmo.

Teorema: Dado el sistema (3.1)-(3.2), supongamos que

$$
r g\left(\frac{\partial g}{\partial z}\right)=m_{1}<m \text {. }
$$

Reordenando si fuera necesario las componentes del campo $g$ y del vector $z$; llamamos

$$
g_{1}=g=\left[\begin{array}{l}
g_{11} \\
g_{12}
\end{array}\right] ; \quad z_{1}=z=\left[\begin{array}{l}
z_{11} \\
z_{12}
\end{array}\right],
$$

con $g_{11}$ a valores en $\mathbb{R}^{m_{1}}, g_{12}$ a valores en $\mathbb{R}^{m-m_{1}}, z_{11} \in \mathbb{R}^{m_{1}}, z_{12} \in \mathbb{R}^{m-m_{1}}$; de modo tal que

$$
r g\left(\frac{\partial g_{11}}{\partial z_{11}}\right)=m_{1}
$$


Suponemos también que

$$
r g\left(\frac{\partial\left(L_{f_{i}} H_{i}\right)}{\partial z_{i 2}}\right)=m_{i+1}<m-\left(m_{1}+\ldots+m_{i}\right), \quad i=1, \ldots, s-2 ;
$$

y

$$
r g\left(\frac{\partial\left(L_{f_{s-1}} H_{s-1}\right)}{\partial z_{(s-1) 2}}\right)=m_{s}=m-\left(m_{1}+\ldots+m_{s-1}\right)
$$

donde definimos recursivamente,

$$
\begin{gathered}
f_{0}=f \\
f_{i}\left(x, w_{11}, \ldots, w_{(i-1) 1}, w_{i 1}, z_{i 2}\right)=f_{i-1}\left(\phi_{i}^{-1}\left(x, w_{11}, \ldots, w_{(i-1) 1}, w_{i 1}, z_{i 2}\right)\right)
\end{gathered}
$$

con

$$
\begin{gathered}
\left(x, w_{11}, w_{21}, \ldots, w_{(i-1) 1}, w_{i 1}, z_{i 2}\right)=\phi_{i}\left(x, w_{11}, w_{21}, \ldots, w_{(i-1) 1}, z_{i 1}, z_{i 2}\right)= \\
=\left(x, w_{11}, \ldots, w_{(i-1) 1}, g_{i 1}\left(x, w_{11}, \ldots, w_{(i-1) 1}, z_{i 1}, z_{i 2}\right), z_{i 2}\right) .
\end{gathered}
$$

En la expresión anterior hemos considerado

$$
g_{i}=\left[\begin{array}{c}
g_{i 1} \\
g_{i 2}
\end{array}\right]=L_{f_{i-1}} H_{i-1}, \quad i=2, \ldots, s-1 ;
$$

donde $H_{i-1}=g_{(i-1) 2} \circ \phi_{i-1}^{-1}, g_{i 1}$ a valores en $\mathbb{R}^{m_{i}}$ y $z_{i 1} \in \mathbb{R}^{m_{i}}$.

Entonces, el sistema (3.1)-(3.2) tiene índice $s$ y su espacio de configuración es

$$
\begin{aligned}
M_{s} & =\left\{\left(x, w_{11}, w_{21}, \ldots, w_{(s-1) 1}, z_{(s-1) 2}\right): w_{11}=0, H_{1}(x)=0, w_{21}=0,\right. \\
H_{2}(x) & \left.=0, \ldots, w_{(s-1) 1}=0, H_{s-1}(x)=0, L_{f_{s-1}} H_{s-1}\left(x, w_{11}, \ldots, w_{(s-1) 1}, z_{(s-1) 2}\right)=0\right\} .
\end{aligned}
$$

Además, se verifica

$f_{s}\left(x, w_{11}, w_{21}, \ldots, w_{(s-1) 1}, z_{(s-1) 2}\right)=f\left(\phi^{-1}\left(x, w_{11}, w_{21}, \ldots, w_{(s-1) 1}, z_{(s-1) 2}\right)\right)$,

siendo

$\left(x, w_{11}, \ldots, w_{(s-1) 1}, z_{(s-1) 2}\right)=\phi\left(x, w_{11}, \ldots, z_{(s-1) 1}, z_{(s-1) 2}\right)=\phi_{s-1} \circ \ldots \circ \phi_{2} \circ \phi_{1}\left(x, z_{11}, z_{12}\right)$. 


\section{3. $\quad \mathrm{SDO}$ con restricciones algebraicas}

En esta Sección analizaremos sistemas diferenciales ordinarios con restricciones algebraicas. Estos sistemas tienen la forma

$$
\begin{aligned}
\dot{w}(t) & =F(w(t)) \\
0 & =G(w(t))
\end{aligned}
$$

con $F$ y $G$ campos vectoriales definidos en un abierto de $\mathbb{R}^{n}$ y a valores en $\mathbb{R}^{n}$ y $\mathbb{R}^{m}$, respectivamente. Los conceptos de regularidad, índice y familia de variedades restringidas establecidos en la sección anterior pueden extenderse para este tipo de sistemas. Para ello definimos,

$$
M_{1}=G^{-1}(0),
$$

que suponemos suave.

Definición 3.7: El sistema (3.15)-(3.16) se llama regular si existe una subvariedad suave $M \subseteq M_{1}$ tal que

$$
\operatorname{Im}\left(\left.F\right|_{M}\right) \subseteq T M
$$

es decir, $F(w) \in T_{w} M, \forall w \in M$. A la variedad $M$ maximal que cumple con esta definición se la llama espacio de configuración del sistema (3.15)-(3.16).

Observación 3.6: Sea $I$ un intervalo real, si (3.15)-(3.16) es regular, entonces $c: I \rightarrow M$ es solución del campo vectorial $v=\left.F\right|_{M}$ sii $j \circ c: I \rightarrow \mathbb{R}^{n}$ es solución de (3.15)-(3.16); siendo $j: M \rightarrow \mathbb{R}^{n}$ la inclusión canónica. Esto significa que el dominio $M$ es el conjunto de condiciones iniciales consistentes del sistema (3.15)-(3.16). Más precisamente, si $w_{0} \in M_{1}$ y $w(t)$ es la solución 
de

$$
\dot{w}(t)=F(w(t)), w(0)=w_{0} ;
$$

para que esta solución satisfaga la restricción (3.16) se debería verificar que $F(w(t)) \in T_{w(t)} M_{1}$, para todo $t \in I$, lo que no ocurre en general. En cambio, si $w_{0} \in M$, entonces $w(t) \in M$, para todo $t \in I$.

Definición 3.8: El conjunto correspondiente al sistema (3.15)-(3.16) es

$$
N=\left\{(w, \dot{w}) \in \mathbb{R}^{2 n}: \dot{w}=F(w), 0=G(w)\right\}
$$

Definición 3.9: Se define la familia de variedades restringidas asociada a (3.15)-(3.16) como:

(i) $M_{0}=\mathbb{R}^{n}$

(ii) $M_{i}=\pi_{1}\left(N \cap T M_{i-1}\right), i=1, \ldots, s$; siendo $s$ el mayor entero tal que $M_{i}$ es variedad suave y $M_{s-1} \neq M_{s}$.

La siguiente proposición establece una forma explícita para los elementos de esta familia.

Proposición 3.6: Sea $\left\{M_{i}\right\}_{i=0}^{s}$ la familia de variedades restringidas asociadas al sistema (3.15)-(3.16), entonces

$$
M_{i}=M_{i-1} \cap\left\{w \in \mathbb{R}^{n}: 0=L_{F}^{i-1} G(w)\right\}, \forall i \geq 1,
$$

siendo $L_{F} G(w)=\frac{\partial G}{\partial w} F(w)$ y $L_{F}^{i} G(w)=L_{F}\left(L_{F}^{i-1} G(w)\right)$. 


\section{Demostración:}

Observar que $(w, \dot{w}) \in N \cap T M_{1}$ sii

$$
\left\{\begin{aligned}
\dot{w} & =F(w) \\
0 & =G(w) \\
0 & =L_{F} G(w)
\end{aligned}\right.
$$

y entonces $M_{2}=M_{1} \cap\left\{w \in \mathbb{R}^{n}: 0=L_{F} G(w)\right\}$.

Razonando inductivamente se tiene que,

$$
M_{i}=M_{i-1} \cap\left\{w \in \mathbb{R}^{n}: 0=L_{F}^{i-1} G(w)\right\} ;
$$

con lo que queda probada la proposición

Definición 3.10: Al entero $s$ se lo llama índice de (3.15)-(3.16).

Observar que si el índice es $s, M=M_{s}$ es el espacio de configuración del sistema.

Concluimos esta Sección con un resultado que garantiza existencia y unicidad de solución para un sistema del tipo (3.15)-(3.16).

Proposición 3.7: Consideramos el sistema (3.15)-(3.16) con condición inicial $w\left(t_{0}\right)=w_{0}$. Supongamos que (3.15) con esta condición inicial tiene solución única. Supongamos también que el sistema (3.15)-(3.16) tiene índice $s$ y que $w_{0} \in M_{s}$.

Entonces, la única solución $c(t)$ de (3.15) definida sobre algún intervalo $I=\left[t_{0}, t_{0}+\delta\right]$, es tal que $c(t) \in M_{s}$, para todo $t \in I$; siendo $M_{s}$ el espacio de configuración de (3.15)-(3.16). 


\section{Demostración:}

Consecuencia inmediata de la definiciones de índice y espacio de configuración.

\subsection{Ejemplos}

En esta Sección presentaremos dos ejemplos simples, y hallaremos en cada caso la familia de variedades restringidas, índice, espacio de configuración y campo vectorial correspondiente.

\section{Ejemplo 1}

Consideramos el siguiente SDAS con trayectorias en $\mathbb{R}^{5}$ :

$$
\begin{aligned}
\dot{x}_{1} & =x_{3} \\
\dot{x}_{2} & =x_{4} \\
\dot{x}_{3} & =-x_{1} x_{4} \\
\dot{x}_{4} & =-x_{2} x_{5}+1 \\
0 & =x_{1}^{2}+x_{2}^{2}-1
\end{aligned}
$$

Usaremos el algoritmo de la Sección 3.2 para determinar la familia de variedades restringidas. En este caso el conjunto correspondiente es

$$
\begin{gathered}
N=\left\{\left(x_{1}, x_{2}, x_{3}, x_{4}, x_{5}, \dot{x}_{1}, \dot{x}_{2}, \dot{x}_{3}, \dot{x}_{4}, \dot{x}_{5}\right): \dot{x}_{1}=x_{3}, \dot{x}_{2}=x_{4},\right. \\
\left.\dot{x}_{3}=-x_{1} x_{4}, \dot{x}_{4}=-x_{2} x_{5}+1, x_{1}^{2}+x_{2}^{2}=1\right\} .
\end{gathered}
$$

- $M_{0}=\mathbb{R}^{5}$

- $M_{1}=\left\{\left(x_{1}, x_{2}, x_{3}, x_{4}, x_{5}\right) \in \mathbb{R}^{5}: x_{1}^{2}+x_{2}^{2}=1\right\}$, que resulta una subvariedad suave de dimensión 4. 
- $M_{2}=\pi_{1}\left(N \cap T M_{1}\right)$

Para determinar $M_{2}$ observar que $\left(x_{1}, x_{2}, x_{3}, x_{4}, x_{5}, \dot{x}_{1}, \dot{x}_{2}, \dot{x}_{3}, \dot{x}_{4}, \dot{x}_{5}\right) \in N \cap T M_{1}$ sii se verifican (3.17)-(3.21) y además

$$
x_{1} x_{3}+x_{2} x_{4}=0
$$

Luego,

$$
M_{2}=M_{1} \cap\left\{\left(x_{1}, x_{2}, x_{3}, x_{4}, x_{5}\right) \in \mathbb{R}^{5}: x_{1} x_{3}+x_{2} x_{4}=0\right\},
$$

subvariedad suave de $\mathbb{R}^{5}$ de dimensión 3 .

- $M_{3}=\pi_{1}\left(N \cap T M_{2}\right)$

En este caso se tiene que $\left(x_{1}, x_{2}, x_{3}, x_{4}, x_{5}, \dot{x}_{1}, \dot{x}_{2}, \dot{x}_{3}, \dot{x}_{4}, \dot{x}_{5}\right) \in N \cap T M_{2}$ sii se verifican (3.17)-(3.22) y

$$
x_{3}^{2}+x_{4}^{2}+x_{2}-x_{5}=0
$$

Entonces,

$$
M_{3}=M_{2} \cap\left\{\left(x_{1}, x_{2}, x_{3}, x_{4}, x_{5}\right) \in \mathbb{R}^{5}: x_{3}^{2}+x_{4}^{2}+x_{2}-x_{5}=0\right\},
$$

subvariedad suave de $\mathbb{R}^{5}$ de dimensión 2 .

- $M_{4}=\pi_{1}\left(N \cap T M_{3}\right)$

Para determinar $M_{4}$, observar que $\left(x_{1}, x_{2}, x_{3}, x_{4}, x_{5}, \dot{x}_{1}, \dot{x}_{2}, \dot{x}_{3}, \dot{x}_{4}, \dot{x}_{5}\right) \in N \cap T M_{3}$ sii se cumplen (3.17)-(3.23); además, diferenciando (3.23) se obtiene:

$$
-2 x_{3} x_{1} x_{5}-2 x_{4} x_{2} x_{5}+3 x_{4}=\dot{x}_{5}
$$

Luego, se tiene que $M_{4}=M_{3}$, y entonces (3.17)-(3.21) es un SDAS regular de índice 3 y espacio de configuración $M=M_{3}$. Su campo vectorial asociado 
tiene dominio $M$ y está determinado por las ecuaciones (3.17)-(3.20) y la ecuación (3.24).

\section{Ejemplo 2}

En este ejemplo analizaremos un sistema diferencial lineal en $\mathbb{R}^{3}$ con una restricción también lineal (es decir un sistema lineal del tipo (3.15)-(3.16)). Este ejemplo se elige de este modo para poder escribir en forma explícita la solución e ilustrar el papel que juegan el campo vectorial y la variedad correspondientes.

$$
\begin{aligned}
\dot{x}_{1} & =x_{2}+x_{3} \\
\dot{x}_{2} & =-x_{1}+x_{3} \\
\dot{x}_{3} & =2 x_{1}+x_{2}-x_{3} \\
0 & =x_{1}+x_{2}-x_{3}
\end{aligned}
$$

La familia de variedades restringidas es:

- $S_{0}=\mathbb{R}^{3}$

- $S_{1}=\left\{\left(x_{1}, x_{2}, x_{3}\right) \in \mathbb{R}^{3}: 0=x_{1}+x_{2}-x_{3}\right\}$

Diferenciando la restricción (3.28), y sustituyendo adecuadamente, se obtiene,

$$
x_{3}-x_{1}=0
$$

Luego,

$$
S_{2}=\left\{\left(x_{1}, x_{2}, x_{3}\right) \in \mathbb{R}^{3}: x_{2}=0, x_{1}=x_{3}\right\}
$$

Además, diferenciando (3.29) para obtener $S_{3}$, no se genera una nueva restricción, se concluye entonces que $S_{3}=S_{2}$. El sistema (3.25)-(3.28) tiene 
índice 2 y su espacio de configuración es $S=S_{2}$.

La solución del sistema diferencial ordinario (3.25)-(3.27) (sin considerar la restricción algebraica), con condición inicial $\left(x_{1}^{0}, x_{2}^{0}, x_{3}^{0}\right)$, es:

$$
\begin{aligned}
& x_{1}(t)=\left(1-e^{-t}+\frac{1}{2} e^{t}\right) x_{1}^{0}+\left(-1+e^{t}\right) x_{2}^{0}+\left(-1+\frac{1}{2} e^{-t}\right) x_{3}^{0} \\
& x_{2}(t)=\left(-1+e^{-t}\right) x_{1}^{0}+x_{2}^{0}+\left(1-e^{-t}\right) x_{3}^{0} \\
& x_{3}(t)=\left(1-\frac{3}{2} e^{-t}+\frac{1}{2} e^{t}\right) x_{1}^{0}+\left(-1+e^{t}\right) x_{2}^{0}+\left(-1+\frac{3}{2} e^{-t}+\frac{1}{2} e^{t}\right) x_{3}^{0}
\end{aligned}
$$

Si por ejemplo, se toma como condición inicial la terna $(1,1,2)$ que satisface la restricción algebraica (3.28), pero no pertenece al espacio de configuración $S$, la solución que se obtiene es

$$
\begin{aligned}
& x_{1}(t)=\frac{1}{2} e^{-t}+\frac{5}{2} e^{t}-2 \\
& x_{2}(t)=-e^{-t}+2 \\
& x_{2}(t)=\frac{3}{2} e^{-t}+\frac{5}{2} e^{t}-2
\end{aligned}
$$

En este caso se puede probar que $x_{1}(t)+x_{2}(t)-x_{3}(t)=0$ sólo si $t=0$.

Si en cambio se toma como condición inicial $(-1,0,-1) \in S$, la solución es

$$
\begin{aligned}
& x_{1}(t)=-e^{t} \\
& x_{2}(t)=0 \\
& x_{3}(t)=-e^{t},
\end{aligned}
$$

luego, las trayectorias del sistema evolucionan sobre $S$, para todo tiempo $t$. 


\section{Capítulo 4}

\section{Sistemas con Perturbación Singular}

\subsection{Introducción}

Las soluciones exactas de ecuaciones diferenciales no-lineales se pueden obtener sólo en un número limitado de casos. En general se debe recurrir a métodos de aproximación.

En este capítulo estudiaremos un método de aproximación de solución para un Sistema con Perturbación Singular (SPS), cuya forma precisa estableceremos más adelante.

\section{Sistemas con Perturbación Regular}

Consideremos primero el sistema perturbado,

$$
\dot{x}(t)=F(t, x(t), \epsilon), \quad x\left(t_{0}\right)=\eta(\epsilon)
$$

donde $x$ es un vector y $\epsilon$ un parámetro de perturbación pequeño. Supongamos el siguiente problema: si bajo ciertas condiciones el sistema (4.1) tiene solución $x(t, \epsilon)$, se quiere obtener una función $\tilde{x}(t, \epsilon)$ tal que la diferencia

$$
x(t, \epsilon)-\tilde{x}(t, \epsilon)
$$


sea pequeña (en alguna norma) en algún dominio de las variables $t$ y $\epsilon$; de modo tal que $\tilde{x}(t, \epsilon)$ se pueda expresar en términos de soluciones de ecuaciones más simples que las del sistema original (4.1). Este problema ha sido completamente resuelto y el método utilizado ha "explotado" esencialmente que $\epsilon$ es pequeño, y luego la aproximación más simple se ha obtenido tomando $\epsilon=0$ en (4.1), que da lugar al sistema nominal

$$
\dot{x}(t)=F(t, x(t), 0), x\left(t_{0}\right)=\eta(0) .
$$

En efecto, suponiendo que,

- el sistema nominal (4.2) tiene única solución $x_{0}(t)$ sobre $\left[t_{0}, t_{1}\right]$, con $x_{0}(t) \in D\left(D\right.$ conjunto conexo y abierto de $\left.\mathbb{R}^{n}\right)$, para todo $t \in\left[t_{0}, t_{1}\right]$,

- $f$ y $\eta$ son ambas dos veces continuamente diferenciables en sus argumentos para $(t, x, \epsilon) \in\left[t_{0}, t_{1}\right] \times D \times\left[-\epsilon_{0}, \epsilon_{0}\right]$,

entonces, para $|\epsilon|$ suficientemente chico, el sistema (4.1) tiene solución única $x(t, \epsilon)$ definida en $\left[t_{0}, t_{1}\right]$; que verifica

$$
\left\|x(t, \epsilon)-x_{0}(t)\right\| \leq k|\epsilon|, \forall|\epsilon|<\epsilon_{1}, \forall t \in\left[t_{0}, t_{1}\right]
$$

para algún $k>0$ y $\epsilon_{1} \leq \epsilon_{0}$. Este resultado se obtiene básicamente por continuidad de la solución con respecto a las condiciones iniciales y a los parámetros, [22].

\section{Sistemas con Perturbación Singular}

Un problema de perturbación diferente se origina cuando el o los parámetros de perturbación que aparecen en el sistema provocan discontinuidades en las soluciones, y es por esto que a esta clase de sistemas se los llama 
de perturbación singular. La esencia de la teoría desarrollada para los SPS es que la discontinuidad de la solución, ocasionada por las perturbaciones singulares, puede evitarse si analizamos el sistema en dos escalas diferentes de tiempo. Este enfoque permite representar al sistema a través de dos subsitemas: el reducido o lento, y el "capa límite" o rápido.

En este capítulo describiremos el comportamiento de las soluciones de un SPS de la forma

$$
\begin{aligned}
\dot{x} & =f(x, z) \\
\epsilon \dot{z} & =g(x, z)
\end{aligned}
$$

con $f: D_{1} \times D_{2} \rightarrow \mathbb{R}^{n}, g: D_{1} \times D_{2} \rightarrow \mathbb{R}^{m}$, donde $D_{1}$ y $D_{2}$ son conjuntos conexos y abiertos de $\mathbb{R}^{n}$ y $\mathbb{R}^{m}$, respectivamente.

Cuando $\epsilon=0$, las propiedades dinámicas del sistema tienen un cambio abrupto pues (4.4) se convierte en la ecuación algebraica o trascendente

$$
0=g(x, z)
$$

y el sistema (4.3)-(4.4) en el SDAS

$$
\begin{aligned}
& \dot{x}=f(x, z) \\
& 0=g(x, z)
\end{aligned}
$$

Supongamos que

$$
r g\left(\frac{\partial g}{\partial z}\right)=r, \quad \text { en un entorno de }\left(x^{*}, z^{*}\right) \in g^{-1}(0) .
$$

Si $r=m$, sabemos por lo visto en la Sección 3.2 que (4.6)-(4.7) es un SDAS de índice 1 , localmente alrededor de $\left(x^{*}, z^{*}\right)$. 
Definición 4.1: Si $r=m$ en (4.8) se dice que (4.3)-(4.4) es un SPS dado en forma standard.

Existen diferentes modelos de SPS que no están en forma standard. En [23] se analiza un SPS no standard lineal y con control, que bajo ciertas hipótesis puede representarse a través de un SDA con control (correspondiente al sistema lento) y de un sistema diferencial con control (correspondiente al sistema rápido). Un análisis similar se realiza en [24], pero en este caso considerando un SPS no-standard, no-lineal y con control. En ambos trabajos se "explota" la descomposición obtenida para diseñar un control compuesto. En [25] se considera una clase particular de SPS no standard, lineales y con control; bajo hipótesis de estabilizabilidad y detectabilidad se extiende la teoría de control por realimentación ya conocida para SPS lineales dados en forma standard. Por otro lado, en [26] se estudia la vinculación entre un SPS no standard y con control (que representa el modelo de un proceso químico) y el SDA con control y de índice mayor que uno que resulta al anular el parámetro de perturbación (que corresponde al sistema lento).

En [27] hemos explorado otra conexión entre los SPS y los SDA. Considerando que el sistema (4.6)-(4.7) es de índice 2 (y luego el SPS (4.3)-(4.4) no está en la forma standard), hemos caracterizado su correspondiente sistema reducido o lento como un SDO con restricciones algebraicas (de la forma dada en la Sección 3.3). Se ha analizado la existencia y unicidad de solución para tal sistema. En [28], considerando nuevamente a (4.6)-(4.7) de índice 2, hemos estudiado las trayectorias de (4.3)-(4.4). En este caso se ha profundizado en el estudio del sistema "capa límite" o rápido, cuya matriz 
jacobiana resulta singular. En particular, para garantizar ciertas hipótesis de estabilidad en este sistema se ha utilizado la Teoría de la Variedad Centro, [29]. Hemos obtenido en este trabajo un resultado de aproximación de las soluciones del SPS, a partir de las soluciones del sistema reducido o lento.

En la Sección 4.2 se dará una breve descripción de los SPS dados en forma standard. Se enunciará un resultado fundamental (Teorema de Tikhonov) que garantiza existencia y unicidad de solución para estos sistemas, y establece también una aproximación de las soluciones. En las secciónes 3 y 4 se detallarán los resultados obtenidos en [27] y [28].

\subsection{SPS en forma standard}

Supondremos en esta Sección que (4.3)-(4.4) es un SPS dado en forma standard en $\left(x^{*}, z^{*}\right) \in g^{-1}(0)$. Entonces, por el TFI, existen entornos $U^{*} \mathrm{y}$ $V^{*}$ de $x^{*}$ y $z^{*}$, respectivamente, y una única función suave $h: U^{*} \rightarrow V^{*}$ tal que:

$$
\left\{(x, z) \in U^{*} \times V^{*}: g(x, z)=0\right\}=\left\{(x, h(x)): x \in U^{*}\right\}
$$

y además, $g(x, h(x))=0, \forall x \in U^{*}$.

Luego, a partir de (4.3)-(4.4), y suponiendo condición inicial

$$
\left(x\left(t_{0}\right), z\left(t_{0}\right)\right)=\left(x_{0}, z_{0}\right)
$$

se obtiene el sistema reducido o lento:

$$
\dot{x}=f(x, h(x)), x\left(t_{0}\right)=x_{0}
$$

Si $\bar{x}(t)$ es la solución de (4.10), entonces podemos obtener $\bar{z}(t)=h(\bar{x}(t))$. Sin embargo, observemos que

$$
\bar{z}\left(t_{0}\right)=h\left(x_{0}\right)
$$


y no necesariamente coincidirá con $z_{0}$. Luego, $\bar{z}(t)$ no es una aproximación uniforme de $z(t, \epsilon)$; se podría esperar, sin embargo, que

$$
z(t, \epsilon)-\bar{z}(t)=O(\epsilon)
$$

sobre algún intervalo $\left[t_{b}, t_{1}\right]$, con $t_{b}>t_{0}$.

Por otro lado, sería razonable que

$$
x(t, \epsilon)-\bar{x}(t)=O(\epsilon) .
$$

uniformememte en $t \in\left[t_{0}, t_{1}\right]$.

Observación 4.1: Si el error $z(t, \epsilon)-\bar{z}(t)$ fuera efectivamente $O(\epsilon)$ sobre $\left[t_{b}, t_{1}\right]$, entonces, durante el intervalo inicial $\left[t_{0}, t_{b}\right]$, debería cumplirse que $z$ se aproxime a $\bar{z}$, y para que esto suceda se deben satisfacer ciertas hipótesis de estabilidad.

Por simplicidad se trabajará en coordenadas $(x, y)$, siendo $y=z-h(x)$. En las nuevas variables $(x, y)$ el sistema (4.3)-(4.4) resulta:

$$
\begin{aligned}
\dot{x} & =f(x, y+h(x)), \\
\epsilon \dot{y} & =g(x, y+h(x))-\epsilon \frac{\partial h}{\partial x} f(x, y+h(x)),
\end{aligned}
$$

con condición inicial

$$
\left(x\left(t_{0}\right), y\left(t_{0}\right)\right)=\left(x_{0}, z_{0}-h\left(x_{0}\right)\right) .
$$

Además, establecemos

$$
\epsilon \frac{d y}{d t}=\frac{d y}{d \tau} \Rightarrow \frac{d \tau}{d t}=\frac{1}{\epsilon}
$$

y utilizamos $\tau=0$ como valor inicial en $t=t_{0}$; entonces la nueva escala de tiempo es

$$
\tau=\frac{t-t_{0}}{\epsilon}
$$


En el tiempo $\tau$ el sistema (4.11)-(4.12) es:

$$
\begin{aligned}
& \left.\frac{d x}{d \tau}=\epsilon f(x), y(\tau)+h(x)\right) \\
& \frac{d y}{d \tau}=g(x, y+h(x))-\epsilon \frac{\partial h}{\partial x} f(x, y+h(x))
\end{aligned}
$$

con condición inicial

$$
(x(0), y(0))=\left(x_{0}, z_{0}-h\left(x_{0}\right)\right)
$$

Si $\epsilon$ tiende a 0 , se obtiene $t=t_{0}$ y $x=x_{0}$; y el sistema (4.14)- (4.15) se transforma en el sistema autónomo:

$$
\begin{aligned}
& \frac{d \hat{x}}{d \tau}=0 \\
& \frac{d \hat{y}}{d \tau}=g\left(x_{0}, \hat{y}+h\left(x_{0}\right)\right)
\end{aligned}
$$

Si suponemos $x_{0} \in U^{*}$, entonces $\hat{y}=0$ es punto de equilibrio de (4.18), pues $g\left(x_{0}, h\left(x_{0}\right)\right)=0$. Al sistema (4.18) se lo llama boundary layer, "capa límite" o rápido.

\section{Teorema de Tikhonov}

El Teorema de Tikhonov establece condiciones suficientes que garantizan la existencia y unicidad de solución para el SPS (4.3)-(4.4) con condición inicial (4.9). Se obtiene además una aproximación para la solución a partir de la solución del sistema nominal que resulta de considerar $\epsilon=0$.

Supongamos que la solución $\bar{x}(t)$ del sistema reducido (4.10) está definido para $t \in\left[t_{0}, t_{1}\right]$, y que $\|\bar{x}(t)\| \leq r_{1}$ sobre $\left[t_{0}, t_{1}\right]$. Se define el conjunto $B_{r}=$ $\left\{x \in \mathbb{R}^{n}:\|x\| \leq r\right\}$, con $r>r_{1}$. Reescribimos el sistema (4.18) como

$$
\frac{d \hat{y}}{d \tau}=g(x, \hat{y}+h(x))
$$


donde los $x \in B_{r}$ son tratados como parámetros fijos.

Definición 4.2: El equilibrio $\hat{y}=0$ del sistema (4.19) es exponencialmente estable, uniformemente en $x \in B_{r}$, si existen constantes positivas $k, \gamma$ y $\rho_{0}$ tales que la solución de (4.19) está definida para todo $\tau \geq 0$ y satisface

$$
\|y(\tau)\| \leq k\|y(0)\| e^{-\gamma \tau}, \quad \forall\|y(0)\|<\rho_{0}, \forall x \in B_{r}, \forall \tau \geq 0 .
$$

Teorema: Consideremos el SPS (4.3)-(4.4) con condición inicial (4.9), y supongamos que se cumple la condición (4.8), es decir, existe $h(x)$ tal que $z=h(x)$, localmente alrededor de $\left(x^{*}, z^{*}\right)$. Supongamos, además, que las siguentes hipótesis se satisfacen para todo $(x, z-h(x)) \in B_{r} \times B_{\rho}$, para cierto $\rho \geq \rho_{0} k$ :

- Las funciones $f$ y $g$, y sus primeras derivadas parciales con respecto a $x$ y $z$ son continuas. La función $h(x)$ y el Jacobiano $\frac{\partial g(x, z)}{\partial z}$ tienen primeras derivadas parciales continuas con respecto a sus argumentos.

- El sistema reducido (4.10) tiene solución única $\bar{x}(t)$ definido sobre $\left[t_{0}, t_{1}\right]$, que cumple $\|\bar{x}(t)\| \leq r_{1}$ para todo $t \in\left[t_{0}, t_{1}\right]$, para cierto $r_{1}<r$.

- El origen del sistema (4.19) es exponencialmente estable, uniformemente en $x$.

Entonces existen constantes positivas $\mu \mathrm{y} \epsilon^{*}$ tal que, para todo $\left\|z_{0}-h\left(x_{0}\right)\right\|<$ $\mu$ y $0<\epsilon<\epsilon^{*}$, el sistema (4.3)-(4.4) con condición inicial (4.9) tiene solución única $(x(t, \epsilon), z(t, \epsilon))$ sobre $\left[t_{0}, t_{1}\right], \mathrm{y}$

$$
\begin{aligned}
x(t, \epsilon)-\bar{x}(t) & =O(\epsilon) \\
z(t, \epsilon)-h(\bar{x}(t))-\hat{y}(t / \epsilon) & =O(\epsilon),
\end{aligned}
$$


uniformemente en $t \in\left[t_{0}, t_{1}\right]$, donde $\hat{y}(\tau)$ es la solución de (4.18). Además, dado $t_{b}>t_{0}$, existe $\epsilon^{* *} \leq \epsilon^{*}$ tal que

$$
z(t, \epsilon)-h(\bar{x}(t))=O(\epsilon)
$$

uniformemente en $t \in\left[t_{b}, t_{1}\right]$, cuando $\epsilon<\epsilon^{* *}$.

Diferentes pruebas de este Teorema pueden encontrarse en [30], [31] y [32].

\subsection{SPS no-standard}

En esta sección consideraremos un SPS no-standard cuyo SDA correspondiente, que se obtiene al anular el parámetro de perturbación, resulta de índice 2 localmente alrededor de $\left(x^{*}, z^{*}\right)$. Como en el caso standard, hallaremos los sistemas reducido y "capa límite". Estudiaremos la existencia y unicidad de solución para el primero y la estabilidad del equilibrio para el segundo.

\section{Sistema reducido o lento}

Queremos analizar cuál será en este caso el sistema reducido, que como en el caso standard deberá depender sólo de las variables lentas del sistema (las variables $x$ ).

Sabemos que al anular el parámetro de perturbación el SPS (4.3)-(4.4) se transforma en el SDAS (4.6)-(4.7). Supongamos que

$$
r g\left(\frac{\partial g}{\partial z}\right)=r<m, \text { en un entorno de }\left(x^{*}, z^{*}\right)
$$

Supongamos también que el sistema (4.6)-(4.7) tiene índice 2. Sabemos entonces, por lo analizado en la Sección 3.2, que existe un cambio de coorde- 
nadas

$$
\left(x, w_{1}, z_{2}\right)=\phi_{1}\left(x, z_{1}, z_{2}\right)
$$

tal que (4.6)-(4.7) en las nuevas coordenadas resulta

$$
\begin{aligned}
\dot{x} & =f_{1}\left(x, w_{1}, z_{2}\right) \\
0 & =w_{1} \\
0 & =H(x) ;
\end{aligned}
$$

siendo $f_{1}\left(x, w_{1}, z_{2}\right)=f\left(\phi_{1}^{-1}\left(x, w_{1}, z_{2}\right)\right)$. Se obtiene entonces el primer sistema reducido en las variables $\left(x, z_{2}\right)$ :

$$
\begin{aligned}
\dot{x} & =\bar{f}_{1}\left(x, z_{2}\right) \\
0 & =H(x)
\end{aligned}
$$

$\operatorname{con} \bar{f}_{1}\left(x, z_{2}\right)=f_{1}\left(x, 0, z_{2}\right)$.

Proposición 4.1: El sistema (4.27)-(4.28) tiene índice 2 en $\left(x^{*}, z_{2}^{*}\right)$.

Demostración:

$$
N^{*}=\left\{\left(x, z_{2}, \dot{x}, \dot{z}_{2}\right): \dot{x}=\bar{f}_{1}\left(x, z_{2}\right), 0=H(x)\right\}
$$

es el conjunto correspondiente a (4.27)-(4.28), y llamamos $\left\{M_{i}^{*}\right\}$ a la familia de variedades restringidas asociada a este sistema. Entonces,

- $M_{1}^{*}=\left\{\left(x, z_{2}\right): 0=H(x)\right\}$

- $M_{2}^{*}=\pi_{1}\left(N \cap T M_{1}^{*}\right)=M_{1}^{*} \cap\left\{\left(x, z_{2}\right): L_{\bar{f}_{1} H}\left(x, z_{2}\right)=0\right\}$,

$\operatorname{con} L_{\bar{f}_{1}} H\left(x, z_{2}\right)=\frac{d H}{d x} \bar{f}_{1}\left(x, z_{2}\right)$.

- $M_{3}^{*}=\pi_{1}\left(N^{*} \cap T M_{2}^{*}\right)$ 
Observar que $\left(x, z_{2}, \dot{x}, \dot{z}_{2}\right) \in N \cap T M_{2}^{*}$ sii

$$
\left\{\begin{aligned}
\dot{x} & =\bar{f}_{1}\left(x, z_{2}\right) \\
0 & =H(x) \\
0 & =L_{\bar{f}_{1}} H\left(x, z_{2}\right) \\
0 & =\frac{\partial L_{\bar{f}_{1}} H\left(x, z_{2}\right)}{\partial x} \bar{f}_{1}\left(x, z_{2}\right)+\frac{\partial L_{\bar{f}_{1}} H\left(x, z_{2}\right)}{\partial z_{2}} \dot{z}_{2}
\end{aligned}\right.
$$

Además, como estamos suponiendo que (4.6)-(4.7) tiene índice 2, se tiene que

$$
r g\left(\frac{\partial\left(L_{\bar{f}_{1}} H\left(x, z_{2}\right)\right)}{\partial z_{2}}\right)=m-r \quad \text { en }\left(x^{*}, z_{2}^{*}\right)
$$

entonces $M_{3}^{*}=M_{2}^{*}$, resultando (4.27)-(4.28) un SDAS también de índice 2, localmente alrededor de $\left(x^{*}, z_{2}^{*}\right)$.

Observar que el sistema reducido obtenido no depende sólo de las variables lentas del SPS, sino también de algunas de las variables rápidas. Ahora bien, bajo la hipótesis (4.29) y aplicando nuevamente el TFI, existen entornos $U_{1} \subset \mathbb{R}^{m}$ y $V_{1} \subset \mathbb{R}^{m-r}$ de $x^{*}$ y $z_{2}^{*}$, respectivamente, y una función suave $\alpha_{2}: U_{1} \rightarrow V_{1}$ de modo tal que,

$$
L_{\bar{f}_{1}} H\left(x, z_{2}\right)=0 \text { sii } z_{2}=\alpha_{2}(x) .
$$

De esta manera, podemos obtener el siguiente sistema reducido, que depende sólo de las variables lentas del SPS:

$$
\begin{aligned}
\dot{x} & =\tilde{f}_{1}(x) \\
0 & =H(x)
\end{aligned}
$$

con $\tilde{f}_{1}(x)=\bar{f}_{1}\left(x, \alpha_{2}(x)\right)$. Se observa que (4.30)-(4.31) es un SDO con restricciones algebraicas de la clase estudiada en la Sección 3.3; además vale el siguiente resultado: 
Proposición 4.2: El sistema (4.30)-(4.31) tiene índice 1 en $x^{*}$.

\section{Demostración:}

Sea $\left\{M_{i}^{* *}\right\}$ la familia de variedades restringidas correspondiente a (4.30)(4.31). Luego se tiene,

$$
M_{1}^{* *}=\{x: H(x)=0\}
$$

y

$$
M_{2}^{* *}=\left\{x \in M_{1}^{*}: \frac{\partial H}{\partial x} \tilde{f}_{1}(x)=0\right\}
$$

Sabemos que el sistema tendrá índice 1 si $M_{1}^{* *}=M_{2}^{* *}$. Ahora bien, utilizando la notación utilizada en la Proposición anterior se tiene que,

$$
x \in M_{1}^{* *} \Rightarrow\left(x, z_{2}\right) \in M_{1}^{*}
$$

y entonces $\left(x, \alpha_{2}(x)\right) \in M_{2}^{*}$ pues $L_{\bar{f}_{1}} H\left(x, \alpha_{2}(x)\right)=0$. Como además $M_{2}^{*} \subset M_{1}^{*}$, vale que $M_{1}^{* *}=M_{2}^{* *}, \mathrm{y}(4.30)-(4.31)$ tiene índice 1.

Corolario: Consideramos el sistema (4.30) con condición inicial $x\left(t_{0}\right)=x_{0}$. Si $H\left(x_{0}\right)=0$, entonces la única solución de (4.30), que estará definida en algún intervalo $I=\left[t_{0}, \delta+t_{0}\right]$, satisface (4.31), para todo $t \in I$.

\section{Demostración:}

Consecuencia de la Proposición 3.7.

\section{Sistema "capa límite" o rápido}

Queremos obtener las ecuaciones del sistema rápido correspondiente a (4.3)-(4.4) con condición inicial $\left(x\left(t_{0}\right), z\left(t_{0}\right)\right)=\left(x_{0}, z_{0}\right)$, para luego estudiar la estabilidad del equilibrio en este sistema. En coordenadas $\left(x, w_{1}, z_{2}\right)$ el 
sistema (4.3)-(4.4) es,

$$
\begin{aligned}
\dot{x}= & f_{1}\left(x, w_{1}, z_{2}\right) \\
\epsilon \dot{w_{1}=} & \epsilon\left(\frac{\partial g_{1}}{\partial x}\left(\phi^{-1}\left(x, w_{1}, z_{2}\right)\right)\right) f_{1}\left(x, w_{1}, z_{2}\right)+ \\
& \frac{\partial g_{1}}{\partial z_{1}}\left(\phi^{-1}\left(x, w_{1}, z_{2}\right)\right) w_{1}+\frac{\partial g_{1}}{\partial z_{2}}\left(\phi^{-1}\left(x, w_{1}, z_{2}\right)\right) \tilde{g}_{2}\left(x, w_{1}\right) \\
\epsilon \dot{z_{2}=} & \tilde{g}_{2}\left(x, w_{1}\right)
\end{aligned}
$$

con condiciones iniciales $\left(x\left(t_{0}\right), w_{1}\left(t_{0}\right), z_{2}\left(t_{0}\right)\right)=\left(x_{0}, w_{10}, z_{20}\right)$, siendo $w_{1}\left(t_{0}\right)=$ $g_{1}\left(x_{0}, z_{10}, z_{20}\right)$ y $\tilde{g}_{2}\left(x, w_{1}\right)=g_{2}\left(\phi^{-1}\left(x, w_{1}, z_{2}\right)\right)$ (que depende sólo de $x$ y $w_{1}$, por lo visto en la Sección 3.2). Observemos además que para obtener la ecuación(4.33) hemos considerado que $w_{1}=g_{1}\left(x, z_{1}, z_{2}\right)$.

En el tiempo $\tau=\frac{t-t_{0}}{\epsilon}$, el sistema (4.32)-(4.34) es:

$$
\begin{aligned}
\frac{d x}{d \tau}= & \epsilon f_{1}\left(x, w_{1}, z_{2}\right), \\
\frac{d w_{1}}{d \tau}= & \epsilon A\left(x, w_{1}, z_{2}\right) f_{1}\left(x, w_{1}, z_{2}\right)+B\left(x, w_{1}, z_{2}\right) w_{1}+ \\
& C\left(x, w_{1}, z_{2}\right) \tilde{g}_{2}\left(x, w_{1}\right), \\
\frac{d z_{2}}{d \tau}= & \tilde{g}_{2}\left(x, w_{1}\right),
\end{aligned}
$$

con condición inicial $\left(x(0), w_{1}(0), z_{2}(0)\right)=\left(x_{0}, w_{10}, z_{20}\right)$; donde,

$$
\begin{aligned}
& A\left(x, w_{1}, z_{2}\right)=\frac{\partial g_{1}}{\partial x}\left(\phi^{-1}\left(x, w_{1}, z_{2}\right)\right) \\
& B\left(x, w_{1}, z_{2}\right)=\frac{\partial g_{1}}{\partial z_{1}}\left(\phi^{-1}\left(x, w_{1}, z_{2}\right)\right) \\
& C\left(x, w_{1}, z_{2}\right)=\frac{\partial g_{1}}{\partial z_{2}}\left(\phi^{-1}\left(x, w_{1}, z_{2}\right)\right)
\end{aligned}
$$

Cuando $\epsilon$ tiende a 0 , se obtiene el sistema,

$$
\begin{aligned}
\frac{d \hat{x}}{d \tau} & =0 \\
\frac{d \hat{w}_{1}}{d \tau} & =B\left(\hat{x}, \hat{w}_{1}, \hat{z}_{2}\right) \hat{w}_{1}+C\left(\hat{x}, \hat{w}_{1}, \hat{z}_{2}\right) \tilde{g}_{2}\left(\hat{x}, \hat{w}_{1}\right), \\
\frac{d \hat{z}_{2}}{d \tau} & =\tilde{g}_{2}\left(\hat{x}, \hat{w}_{1}\right),
\end{aligned}
$$


con condición inicial $\left(\hat{x}(0), \hat{w}_{1}(0), \hat{z}_{2}(0)\right)=\left(x_{0}, w_{10}, z_{20}\right)$.

Observación 4.3: De (4.38) se tiene que $\hat{x}(\tau)=x_{0}$, luego (4.38)-(4.40) es equivalente a:

$$
\begin{aligned}
\frac{d \hat{w}_{1}}{d \tau} & =B\left(x_{0}, \hat{w}_{1}, \hat{z}_{2}\right) \hat{w}_{1}+C\left(x_{0}, \hat{w}_{1}, \hat{z}_{2}\right) \tilde{g}_{2}\left(x_{0}, \hat{w}_{1}\right), \\
\frac{d \hat{z}_{2}}{d \tau} & =\tilde{g}_{2}\left(x_{0}, \hat{w}_{1}\right)
\end{aligned}
$$

con condición inicial $\left(\hat{w}_{1}(0), \hat{z}_{2}(0)\right)=\left(w_{10}, z_{20}\right)$.

El sistema (4.41)-(4.42) es el sistema rápido correspondiente a (4.3)(4.4). Todos los puntos de la forma $\left(0, z_{2}^{e}\right)$ son puntos de equilibrio del sistema rápido (4.41)-(4.42), cualquiera sea $z_{2}^{e}$, pues supondremos de aquí en más que $\tilde{g}_{2}\left(x_{0}, 0\right)=H\left(x_{0}\right)=0$. Para estudiar la estabilidad de cualquiera de estos puntos calculamos la matriz jacobiana del sistema, la evaluamos en el punto de equilibrio, y obtenemos:

$$
J=\left[\begin{array}{cc}
B\left(x_{0}, 0, z_{2}^{e}\right)+C\left(x_{0}, 0, z_{2}^{e}\right) \frac{\partial \tilde{g}_{2}}{\partial \hat{w}_{1}}\left(x_{0}, 0\right) & 0 \\
\frac{\partial \tilde{g}_{2}}{\partial \hat{w}_{1}}\left(x_{0}, 0\right) & 0
\end{array}\right] .
$$

Si llamamos

$$
\begin{gathered}
A_{1}=B\left(x_{0}, 0, z_{2}^{e}\right)+C\left(x_{0}, 0, z_{2}^{e}\right) \frac{\partial \tilde{g}_{2}}{\partial \hat{w}_{1}}\left(x_{0}, 0\right), \\
A_{2}=\frac{\partial \tilde{g}_{2}}{\partial \hat{w}_{1}}\left(x_{0}, 0\right),
\end{gathered}
$$

el sistema (4.41)-(4.42) puede escribirse como

$$
\begin{aligned}
& \frac{d \hat{w}_{1}}{d \tau}=A_{1} \hat{w}_{1}+F_{1}\left(x_{0}, \hat{w}_{1}, \hat{z}_{2}\right) \\
& \frac{d \hat{z}_{2}}{d \tau}=A_{2} \hat{w}_{1}+F_{2}\left(x_{0}, \hat{w}_{1}\right) ;
\end{aligned}
$$

donde 


$$
\begin{gathered}
F_{1}\left(x_{0}, \hat{w}_{1}, \hat{z}_{2}\right)=B\left(x_{0}, \hat{w}_{1}, \hat{z}_{2}\right) \hat{w}_{1}+C\left(x_{0}, \hat{w}_{1}, \hat{z}_{2}\right) \tilde{g}_{2}\left(x_{0}, \hat{w}_{1}\right)-A_{1} \hat{w}_{1}, \\
F_{2}\left(x_{0}, \hat{w}_{1}\right)=\tilde{g}_{2}\left(x_{0}, \hat{w}_{1}\right)-A_{2} \hat{w}_{1} .
\end{gathered}
$$

Consideramos ahora una transformación de similaridad $T$ de modo tal que

$$
M=\left[\begin{array}{cc}
B_{1} & 0 \\
0 & 0
\end{array}\right]=T^{-1}\left[\begin{array}{ll}
A_{1} & 0 \\
A_{2} & 0
\end{array}\right] T,
$$

es la forma canónica de Jordan correspondiente a

$$
\left[\begin{array}{ll}
A_{1} & 0 \\
A_{2} & 0
\end{array}\right]
$$

Luego de aplicar esta transformación de similaridad, en las nuevas coordenadas (que seguiremos llamando $\left(\hat{w}_{1}, \hat{z}_{2}\right)$ ), el sistema rápido resulta:

$$
\begin{aligned}
& \frac{d \hat{w}_{1}}{d \tau}=B_{1} \hat{w}_{1}+\tilde{F}_{1}\left(x_{0}, \hat{w}_{1}, \hat{z}_{2}\right) \\
& \frac{d \hat{z}_{2}}{d \tau}=\tilde{F}_{2}\left(x_{0}, \hat{w}_{1}\right)
\end{aligned}
$$

donde $\tilde{F}_{1}$ y $\tilde{F}_{2}$ son las expresiones de $F_{1}$ y $F_{2}$ en las nuevas coordenadas. Además mediante cálculos sencillos se prueba que

$$
\begin{aligned}
\tilde{F}_{1}\left(x_{0}, 0, z_{2}^{e}\right) & =0, \quad \tilde{F}_{2}\left(x_{0}, 0, z_{2}^{e}\right)=0 \\
\frac{\partial \tilde{F}_{1}}{\partial\left(\hat{w}_{1}, \hat{z}_{2}\right)}\left(x_{0}, 0, z_{2}^{e}\right) & =0, \frac{\partial \tilde{F}_{2}}{\partial\left(\hat{w}_{1}, \hat{z}_{2}\right)}\left(x_{0}, 0, z_{2}^{e}\right)=0 .
\end{aligned}
$$

Como $M$ es singular, con $m-r$ autovalores nulos, recurriremos a la Teoría de la Variedad Centro para estudiar la estabilidad del equilibrio. En el Apéndice A se establecen algunas definiciones y resultados vinculados a esta Teoría. Como resultado de aplicar al sistema (4.45)-(4.46) estos resultados se tiene,

Proposition 4.3: $\hat{w}_{1}=0$ es una variedad centro para el sistema (4.45)(4.46) que pasa por el punto de equilibrio $\left(0, z_{2}^{e}\right)$. 


\section{Demostración:}

En general, una variedad centro para (4.45)-(4.46) por un equilibrio $\left(w_{1}^{e}, z_{2}^{e}\right)$ es una función suave $\hat{w}_{1}=\gamma\left(\hat{z}_{2}\right)$ que verifica:

1. $\gamma\left(z_{2}^{e}\right)=w_{1}^{e}$

2. $\frac{\partial \gamma}{\partial \hat{z}_{2}}\left(z_{2}^{e}\right)=0$

3. La variedad es invariante para el sistema, es decir,

$$
\frac{d \hat{w}_{1}}{d \tau}=B_{1} \gamma\left(\hat{z}_{2}\right)+\tilde{F}_{1}\left(x_{0}, \gamma\left(\hat{z}_{2}\right), \hat{z}_{2}\right)=\frac{d \gamma}{d \hat{z}_{2}} \tilde{F}_{2}\left(x_{0}, \gamma\left(\hat{z}_{2}\right)\right)
$$

En este caso, para la variedad $\hat{w}_{1}=0$ y algún punto de equilibrio de la forma $\left(w_{1}^{e}, z_{2}^{e}\right)=\left(0, z_{2}^{e}\right)$, las condiciones 1 y 2 se verifican trivialmente. Para probar la condición 3 , observar que la dinámica sobre la variedad $\hat{w}_{1}=0$ es,

$$
\frac{d \hat{w}_{1}}{d \tau}=\tilde{F}_{1}\left(x_{0}, 0, \hat{z}_{2}\right)=0
$$

lo que prueba la invariancia; luego concluimos que $\hat{w}_{1}=0$ es una variedad centro para (4.45)-(4.46) por el punto de equilibrio $\left(0, z_{2}^{e}\right)$.

Observación 4.4: Por Teorema A-2 (parte a)), la estabilidad del equilibrio de (4.45)-(4.46) está determinada por la estabilidad de $u^{e}=z_{2}^{e}$ en la dinámica

$$
\frac{d u}{d \tau}=\tilde{F}_{2}\left(x_{0}, 0\right)
$$

Como en este caso $\tilde{F}_{2}$ no depende de $u$, y $\tilde{F}_{2}\left(x_{0}, 0\right)=0$, entonces $\left(0, z_{2}^{e}\right)$ es un punto de equilibrio estable del sistema (4.45)-(4.46).

Teniendo en cuenta la Proposición 4.3 y la observación 4.4, aplicamos nuevamente el Teorema A-2 (parte b)). Entonces, existe una constante $\rho>0$ y 
una solución $u_{0}$ de

$$
\frac{d u}{d \tau}=0
$$

tal que, si $\tau \longrightarrow \infty$,

$$
\begin{aligned}
\hat{w}_{1}(\tau) & =O\left(e^{-\rho \tau}\right) \\
\hat{z}_{2}(\tau)-u_{0} & =O\left(e^{-\rho \tau}\right)
\end{aligned}
$$

Concluimos entonces,

Proposition 4.4: El equilibrio $\left(\hat{w}_{1}^{e}, \hat{z}_{2}^{e}\right)=\left(0, u_{0}\right)$ de (4.45)-(4.46) es asintóticamente estable.

\subsection{Aproximación de trayectorias}

En esta Sección utilizaremos el resultado de la Proposición 4.4 para probar que las trayectorias del sistema (4.35)-(4.37) pueden aproximarse con las del sistema (4.38)-(4.40), cuando $\epsilon$ tiende a 0 , uniformemente en $\tau \geq 0$. Para probar este resultado utilizaremos la Teoría de Perturbación Regular para ecuaciones diferenciales ordinarias, pues en este caso se tienen funciones que dependen en forma suave del parámetro de perturbación.

Entonces, (4.35)-(4.37) es el sistema perturbado y (4.38)-(4.40) el sistema nominal o no perturbado.

Si llamamos,

$$
\begin{gathered}
X=\left[\begin{array}{lll}
x & w_{1} & z_{2}
\end{array}\right]^{\prime}, \quad \hat{X}=\left[\begin{array}{lll}
\hat{x} & \hat{w}_{1} & \hat{z}_{2}
\end{array}\right]^{\prime}, \\
F(X, \epsilon)=\left[\begin{array}{lll}
\epsilon & f_{1}\left(x, w_{1}, z_{2}\right) & \epsilon A\left(x, w_{1}, z_{2}\right) f_{1}\left(x, w_{1}, z_{2}\right)+B\left(x, w_{1}, z_{2}\right) w_{1}+ \\
C\left(x, w_{1}, z_{2}\right) \tilde{g}_{2}\left(x, w_{1}\right) & \tilde{g}_{2}\left(x, w_{1}\right)
\end{array}\right]^{\prime}, \\
\hat{F}(\hat{X})=\left[\begin{array}{lll}
0 & B\left(\hat{x}, \hat{w}_{1} \hat{z}_{2}\right) \hat{w}_{1}+C\left(\hat{x}, \hat{w}_{1} \hat{z}_{2}\right) \tilde{g}_{2}\left(\hat{x}, \hat{w}_{1}\right) & \left.\tilde{g}_{2}\left(\hat{x}, \hat{w}_{1}\right)\right]^{\prime},
\end{array}\right.
\end{gathered}
$$


entonces podemos establecer el siguiente resultado de aproximación válido en el intervalo infinito, (ver [30]).

Proposición 4.5: Supongamos que

- $F$ está definida para $(X, \epsilon) \in D \times\left[0, \epsilon_{0}\right]$, con $D$ conexo y abierto.

- $X^{e}=\left(x_{0}, 0, u_{0}\right)$ es punto de equilibrio asintóticamente estable del sistema nominal.

Entonces,

$$
X(\tau, \epsilon)-\hat{X}(\tau)
$$

tiende a 0 cuando $\epsilon \longrightarrow 0$, uniformemente en $\tau$, para todo $\tau \geq 0$; siendo $X(\tau, \epsilon)$ la solución de

$$
\frac{d X}{d \tau}=F(X, \epsilon), \quad X(0)=X_{0},
$$

y $\hat{X}(\tau)$ solución de

$$
\frac{d \hat{X}}{d \tau}=\hat{F}(\hat{X}), \quad \hat{X}(0)=X_{0}
$$

Observación 4.5: la segunda hipótesis de esta proposición vale como consecuencia de la Proposición 4.4.

Para terminar esta Sección enunciaremos y probaremos una extensión del Teorema de Tikhonov para el caso en que el SPS (4.3)-(4.4) es no-standard localmente $\left(x^{*}, z^{*}\right) \in g^{-1}(0)$. 


\section{Resultado principal}

El siguiente resultado garantiza existencia y unicidad de solución para el sistema (4.3)-(4.4) con condición inicial $\left(x\left(t_{0}\right), z\left(t_{0}\right)\right)=\left(x_{0}, z_{0}\right)$. Se obtiene también una aproximación de la solución del sistema a partir de la solución del SDAS (4.6)-(4.7) que se supondrá de índice 2 en $\left(x^{*}, z^{*}\right)$.

Teorema: Consideramos el SPS (4.3)-(4.4), pero en coordenadas $\left(x, w_{1}, z_{2}\right)$, es decir en la forma (4.32)-(4.34), con condición inicial $\left(x\left(t_{0}\right), w_{1}\left(t_{0}\right), z_{2}\left(t_{0}\right)\right)=$ $\left(x_{0}, w_{10}, z_{20}\right)$.

Supongamos que

(i) El SDAS que resulta de hacer $\epsilon=0$ tiene índice 2 localmente en algún punto $\left(x^{*}, z^{*}\right) \in g^{-1}(0)$.

(ii) El sistema reducido que resulta en este caso, es decir el sistema de ecuaciones (4.30)-(4.31), es tal que $H\left(x_{0}\right)=0$; y entonces admite única solución $x_{r}(t)$ en algún intervalo $\left[t_{0}, t_{1}\right]$ (consecuencia del Corolario de la Proposición 4.2).

(iii) Considerando el sistema rápido (4.45)-(4.46), $B_{1}$ es una matriz con todos sus autovalores de parte real negativa.

Entonces, existe una constante $\mu>0$ tal que si $\left\|\left(w_{10}, z_{20}-\alpha_{2}\left(x_{0}\right)\right)\right\|<\mu$, el sistema (4.32)-(4.34) tiene solución única $\left(x(t, \epsilon), w_{1}(t, \epsilon), z_{2}(t, \epsilon)\right)$ y se verifica

$$
\begin{gathered}
x(t, \epsilon)-x_{r}(t) \rightarrow 0 \\
\left(w_{1}(t, \epsilon), z_{2}(t, \epsilon)-\alpha_{2}\left(x_{r}(t)\right)\right) \rightarrow\left(\hat{w}_{1}\left(\frac{t-t_{0}}{\epsilon}\right), \hat{z}_{2}\left(\frac{t-t_{0}}{\epsilon}\right)-\alpha_{2}\left(x_{0}\right)\right)
\end{gathered}
$$


cuando $\epsilon \rightarrow 0$, uniformemente en $t \in\left[t_{0}, t_{1}\right]$; siendo $\left(\hat{w}_{1}(\tau), \hat{z}_{2}(\tau)\right)$ la solución de (4.41)-(4.42).

Además, dado $t_{b}>t_{0}$,

$$
w_{1}(t, \epsilon) \longrightarrow 0
$$

cuando $\epsilon \rightarrow 0$, uniformemente en $t \in\left[t_{b}, t_{1}\right]$.

\section{Demostración:}

Las hipótesis generales supuestas en el Capítulo 2 (Preliminares) garantizan existencia y unicidad de solución para el sistema (4.32)-(4.34).

Nos ocuparemos entonces de la aproximación de las trayectorias de este sistema con las del sistema reducido,

$$
\begin{aligned}
\dot{x}_{r} & =\tilde{f}_{1}\left(x_{r}\right) \\
0 & =H\left(x_{r}\right)
\end{aligned}
$$

Analizamos en dos partes: el error para $x$ y el error para $\left(w_{1}, z_{2}\right)$.

\section{Error para $x$ :}

Si definimos $e_{x}(t, \epsilon)=x(t, \epsilon)-x_{r}(t)$ entonces,

$$
\begin{gathered}
e_{x}(t, \epsilon)=\int_{t_{0}}^{t}\left[f_{1}\left(x(s, \epsilon), w_{1}(s, \epsilon), z_{2}(s, \epsilon)\right)-\tilde{f}_{1}\left(x_{r}(s)\right)\right] d s= \\
\int_{t_{0}}^{t}\left[f_{1}\left(x(s, \epsilon), w_{1}(s, \epsilon), z_{2}(z, \epsilon)\right)-f_{1}\left(x_{r}(s), 0, \alpha_{2}\left(x_{r}(s)\right)\right)\right] d s= \\
\int_{t_{0}}^{t}\left[f_{1}\left(x(s, \epsilon), w_{1}(s, \epsilon), z_{2}(s, \epsilon)\right)-f_{1}\left(x_{r}(s), w_{1}(s, \epsilon), z_{2}(s, \epsilon)\right)\right] d s+ \\
+\int_{t_{0}}^{t}\left[f_{1}\left(x_{r}(s), w_{1}(s, \epsilon), z_{2}(s, \epsilon)\right)-f_{1}\left(x_{r}(s), 0, \alpha_{2}(x(s, \epsilon))\right)\right] d s+ \\
+\int_{t_{0}}^{t}\left[f_{1}\left(x_{r}(s), 0, \alpha_{2}(x(s, \epsilon))\right)-f_{1}\left(x_{r}(s), 0, \alpha_{2}\left(x_{r}(s)\right)\right)\right] d s
\end{gathered}
$$


y en consecuencia, suponiendo que $f_{1}$ y $\alpha_{2}$ son Lipschitz en sus argumentos,

$$
\begin{gathered}
\left\|e_{x}(t, \epsilon)\right\| \leq \int_{t_{0}}^{t} k_{1}\left\|x(s, \epsilon)-x_{r}(s)\right\| d s+ \\
\int_{t_{0}}^{t} k_{2}\left\|\left(w_{1}(s, \epsilon), z_{2}(s, \epsilon)-\alpha_{2}(x(s, \epsilon))\right)\right\| d s+\int_{t_{0}}^{t} k_{3}\left\|x(s, \epsilon)-x_{r}(s)\right\| d s .
\end{gathered}
$$

Para obtener una cota para

$$
\left(w_{1}(s, \epsilon), z_{2}(s, \epsilon)-\alpha_{2}(x(s, \epsilon))\right)
$$

trabajaremos, por simplicidad, en el tiempo $\tau$. Entonces,

$$
\begin{gathered}
\left\|\left(w_{1}(\tau, \epsilon), z_{2}(\tau, \epsilon)-\alpha_{2}(x(\tau, \epsilon))\right)\right\| \leq \\
\left\|\left(w_{1}(\tau, \epsilon)-\hat{w}_{1}(\tau), z_{2}(\tau, \epsilon)-\hat{z}_{2}(\tau)\right)\right\|+\left\|\left(\hat{w}_{1}(\tau), \hat{z}_{2}(\tau)-\alpha_{2}\left(x_{0}\right)\right)\right\|+ \\
\left.+\| \alpha_{2}(x(\tau, \epsilon))-\alpha_{2}\left(x_{0}\right)\right) \|
\end{gathered}
$$

Por Proposición 4.5, dado $\delta_{1}>0$, existe $\epsilon_{1}>0$ tal que

$$
\left\|\left(w_{1}(\tau, \epsilon)-\hat{w}_{1}(\tau), z_{2}(\tau, \epsilon)-\hat{z}_{2}(\tau)\right)\right\|<\frac{\delta_{1}}{3}, \forall \tau \geq 0, \forall \epsilon<\epsilon_{1} .
$$

Por lo visto en la Sección $4.3,\left(0, \alpha_{2}\left(x_{0}\right)\right)$ es un punto de equilibrio estable del sistema (4.45)-(4.46); entonces existe una constante $\mu>0$ tal que, si $\left\|\left(w_{10}, z_{20}-\alpha_{2}\left(x_{0}\right)\right)\right\|<\mu$, se cumple que

$$
\left\|\left(\hat{w}_{1}(\tau), \hat{z}_{2}(\tau)-\alpha_{2}\left(x_{0}\right)\right)\right\|<\frac{\delta_{1}}{3}, \forall \tau \geq 0 .
$$

Además, existe una constante $K>0$, tal que

$$
\left.\| \alpha_{2}(x(\tau, \epsilon))-\alpha_{2}\left(x_{0}\right)\right)\|\leq K\| x(\tau, \epsilon)-x_{0} \|
$$

pues $\alpha_{2}$ es Lipschitz en $x$. Además, dado $\delta_{2}=\frac{\delta_{1}}{3 K}$, existe $\epsilon_{2}>0$ tal que $\left\|x(\tau, \epsilon)-x_{0}\right\|<\delta_{2}$, para todo $\epsilon<\epsilon_{2}$ (nuevamente se aplica la Proposición 
$4.5)$.

Entonces, concluimos que

$$
\left\|\alpha_{2}(x(\tau, \epsilon))-\alpha_{2}\left(x_{0}\right)\right\|<\frac{\delta_{1}}{3}, \quad \forall \epsilon<\epsilon_{2}, \quad \forall \tau \geq 0
$$

Finalmente, si elegimos $\epsilon^{*}=\operatorname{mín}\left(\epsilon_{1}, \epsilon_{2}\right)$, obtenemos que,

$$
\left\|\left(w_{1}(\tau, \epsilon), z_{2}(\tau, \epsilon)-\alpha_{2}(x(\tau, \epsilon))\right)\right\|<\delta_{1}, \quad \forall \epsilon<\epsilon^{*}, \quad \forall \tau \geq 0
$$

o equivalentemente,

$$
\left\|\left(w_{1}(s, \epsilon), z_{2}(s, \epsilon)-\alpha_{2}(x(s, \epsilon))\right)\right\|<\delta_{1}, \quad \forall \epsilon<\epsilon^{*}, \quad \forall s \in\left[t_{0}, t_{1}\right] .
$$

Ahora, dado $\delta>0$, se tiene,

$$
\left\|e_{x}(t, \epsilon)\right\| \leq \int_{t_{0}}^{t} K_{1}\left\|e_{x}(s, \epsilon)\right\| d s+k_{2} \delta_{1}\left(t_{1}-t_{0}\right)
$$

$\operatorname{con} K_{1}=\min \left(k_{1}, k_{3}\right)$.

Por la desigualdad de Gronwall, concluimos que

$$
\left\|e_{x}(t, \epsilon)\right\| \leq k_{2} \delta_{1}\left(t_{1}-t_{0}\right) e^{K_{1}\left(t_{1}-t_{0}\right)}, \quad \forall t \in\left[t_{0}, t_{1}\right] .
$$

Entonces, si elegimos

$$
\delta_{1}=\frac{\delta}{k_{2}\left(t_{1}-t_{0}\right) e^{K_{1}\left(t_{1}-t_{0}\right)}}
$$

resulta,

$$
\left\|e_{x}(t, \epsilon)\right\| \leq \delta, \quad \forall \epsilon<\epsilon^{*}=\min \left(\epsilon_{1}, \epsilon_{2}\right),
$$

uniformemente en $t \in\left[t_{0}, t_{1}\right]$; lo que prueba (4.49). 
Error para $\left(w_{1}, z_{2}\right)$ :

Para probar (4.50) trabajaremos nuevamente en el tiempo $\tau$.

$$
\begin{gathered}
\left(w_{1}(\tau, \epsilon), z_{2}(\tau, \epsilon)-\alpha_{2}\left(x_{r}(\tau)\right)\right)-\left(\hat{w}_{1}(\tau), \hat{z}_{2}(\tau)-\alpha_{2}\left(x_{0}\right)\right)= \\
\left(w_{1}(\tau, \epsilon)-\hat{w}_{1}(\tau), z_{2}(\tau, \epsilon)-\hat{z}_{2}(\tau)\right)+\left(0, \alpha_{2}(x(\tau, \epsilon))-\alpha_{2}\left(x_{r}(\tau)\right)\right)+ \\
+\left(0, \alpha_{2}\left(x_{0}\right)-\alpha_{2}(x(\tau, \epsilon))\right)
\end{gathered}
$$

que tiende a 0 , para $\epsilon$ suficientemente paqueño, uniformemente en $\tau \geq 0$. Más precisamente, para que cada término de la suma sea suficientemente pequeño se ha utilizado: para el primer término la Proposición 4.5; para el segundo término que $\alpha_{2}$ es Lipschitz y la cota (4.49) probada antes; para el tercer término que $\alpha_{2}$ es Lipschitz y nuevamente la Proposición 4.5. Así queda probado (4.50). Para probar (4.51), consideremos $t_{b}>t_{0}$ y $\delta>0$. Luego, existe $\epsilon_{3}>0$ tal que,

$$
\left\|w_{1}(t, \epsilon)-\hat{w}_{1}\left(\frac{t-t_{0}}{\epsilon}\right)\right\|<\delta / 2
$$

para todo $\epsilon<\epsilon_{3}$ y todo $t \in\left[t_{0}, t_{1}\right]$.

Entonces,

$$
\left\|w_{1}(t, \epsilon)\right\|<\delta / 2+\left\|\hat{w}_{1}\left(\frac{t-t_{0}}{\epsilon}\right)\right\|
$$

e invocando el Lema del Apéndice A, si $\left\|w_{10}\right\|$ es suficientemente pequeño, existen constantes positivas $c_{1}$ y $\beta$ tales que,

$$
\left\|\hat{w}_{1}\left(\frac{t-t_{0}}{\epsilon}\right)\right\| \leq c_{1} e^{-\beta\left(\frac{t-t_{0}}{\epsilon}\right)}\left\|w_{10}\right\| .
$$

Como

$$
e^{-\beta\left(\frac{t-t_{0}}{\epsilon}\right)} \leq \epsilon, \forall \beta\left(t-t_{0}\right) \geq \epsilon \ln \left(\frac{1}{\epsilon}\right)
$$


entonces (4.54) será $O(\epsilon)$ uniformemente en $\left[t_{b}, t_{1}\right]$ si $\epsilon$ es suficientemente paqueño para satisfacer

$$
\epsilon \ln \left(\frac{1}{\epsilon}\right) \leq \beta\left(t_{b}-t_{0}\right)
$$

Más precisamente, existen constantes $\lambda>0$ y $\epsilon_{4}>0$ tales que,

$$
\left\|\hat{w}_{1}\left(\frac{t-t_{0}}{\epsilon}\right)\right\| \leq \lambda \epsilon
$$

para todo $\epsilon<\epsilon_{4}$, uniformemente en $t \in\left[t_{b}, t_{1}\right]$.

Ahora podemos elegir $\epsilon_{5} \leq \epsilon_{4}$ tal que $\lambda \epsilon<\delta / 2$, para todo $\epsilon<\epsilon_{5}$; entonces

$$
\left\|\hat{w}_{1}\left(\frac{t-t_{0}}{\epsilon}\right)\right\|<\delta / 2
$$

para todo $\epsilon<\epsilon_{5}$, uniformemente en $t \in\left[t_{b}, t_{1}\right]$.

Finalmente, si $\epsilon^{* *}=\operatorname{mín}\left(\epsilon_{3}, \epsilon_{5}\right)$, se cumple que

$$
\left\|w_{1}(t, \epsilon)\right\|<\delta, \quad \forall \epsilon<\epsilon^{* *}
$$

uniformemente en $t \in\left[t_{b}, t_{1}\right]$; y se prueba (4.51).

\section{Ejemplo}

En esta Sección analizaremos el comportamiento de las trayectorias del siguiente SPS no-standard,

$$
\begin{aligned}
\dot{x_{1}} & =x_{2}^{2}+z_{1}-z_{2} \\
\dot{x_{2}} & =x_{1}+z_{1} \\
\epsilon \dot{z}_{1} & =-z_{1}+x_{1} x_{2} \\
\epsilon \dot{z}_{2} & =x_{1}+x_{2}
\end{aligned}
$$


Cuando $\epsilon=0$, obtenemos el SDAS,

$$
\begin{aligned}
\dot{x_{1}} & =x_{2}^{2}+z_{1}-z_{2} \\
\dot{x_{2}} & =x_{1}+z_{1} \\
0 & =-z_{1}+x_{1} x_{2} \\
0 & =x_{1}+x_{2}
\end{aligned}
$$

Si llamamos,

$g\left(x_{1}, x_{2}, z_{1}, z_{2}\right)=\left[g_{1}\left(x_{1}, x_{2}, z_{1}, z_{2}\right) \quad g_{2}\left(x_{1}, x_{2}, z_{1}, z_{2}\right]^{\prime}=\left[\begin{array}{ll}-z_{1}+x_{1} x_{2} & x_{1}+x_{2}\end{array}\right]^{\prime}\right.$, entonces,

$$
r g\left(\frac{\partial g}{\partial\left(z_{1}, z_{2}\right)}\right)=1, \quad \text { para todo }\left(x_{1}, x_{2}, z_{1}, z_{2}\right)
$$

además,

$$
r g\left(\frac{\partial g_{1}}{\partial z_{1}}\right)=1
$$

En este caso trabajaremos directamente en las coordenadas $\left(x_{1}, x_{2}, z_{1}, z_{2}\right)$, pues a partir de la restricción algebraica (4.61) obtenemos,

$$
z_{1}=\alpha_{1}\left(x_{1}, x_{2}\right)=x_{1} x_{2} .
$$

Por otro lado, llamando

$$
\begin{gathered}
f\left(x_{1}, x_{2}, z_{1}, z_{2}\right)=\left[\begin{array}{cc}
x_{2}^{2}+z_{1}-z_{2} & x_{1}+z_{1}
\end{array}\right]^{\prime}, H(x)=x_{1}+x_{2} \\
\left.f_{1}\left(x_{1}, x_{2},, z_{2}\right)=f\left(x_{1}, x_{2}, \alpha_{1}\left(x_{1}, x_{2}, z_{2}\right), z_{2}\right)\right)
\end{gathered}
$$

entonces

$$
L_{f_{1}} H\left(x_{1}, x_{2}, z_{2}\right)=x_{2}^{2}+2 x_{1} x_{2}+x_{1}-z_{2},
$$

y entonces el sistema (4.59)-(4.62) tiene índice 2 en todo punto, pues

$$
r g\left(\frac{\partial\left(L_{f_{1}} H\right)}{\partial z_{2}}\right)=1 .
$$


Además, de la expresión

$$
L_{f_{1}} H\left(x_{1}, x_{2}, z_{2}\right)=0
$$

se obtiene

$$
z_{2}=\alpha_{2}\left(x_{1}, x_{2}\right)=x_{2}^{2}+2 x_{1} x_{2}+x_{1}
$$

Luego, el sistema reducido correspondiente es en este caso,

$$
\begin{aligned}
\dot{x}_{1 r} & =x_{1 r} x_{2 r}-x_{1 r} \\
\dot{x}_{2 r} & =-x_{1 r} x_{2 r}+x_{1 r} \\
0 & =x_{1 r}+x_{2 r}
\end{aligned}
$$

que resulta un SDAS de índice 1 (como habíamos visto en la Proposición $4.2)$.

Si expresamos al sistema (4.55)-(4.58) en el tiempo $\tau=\frac{t-t_{0}}{\epsilon}$ tenemos,

$$
\begin{aligned}
& \frac{d x_{1}}{d \tau}=\epsilon\left(x_{2}^{2}+z_{1}-z_{2}\right) \\
& \frac{d x_{2}}{d \tau}=\epsilon\left(x_{1}+z_{1}\right) \\
& \frac{d z_{1}}{d \tau}=-z_{1}+x_{1} x_{2} \\
& \frac{d z_{2}}{d \tau}=x_{1}+x_{2},
\end{aligned}
$$

Cuando $\epsilon$ tiende a 0 , y suponiendo condiciones iniciales $\left(x_{10}, x_{20}, z_{10}, z_{20}\right)$, se obtiene el sistema "capa límiteçorrespondiente,

$$
\begin{aligned}
& \frac{d \hat{z}_{1}}{d \tau}=-\hat{z}_{1}+x_{10} x_{20} \\
& \frac{d \hat{z}_{2}}{d \tau}=x_{10}+x_{20}
\end{aligned}
$$

Como en las hipótesis del Teorema se debe satisfacer:

$$
H\left(x_{10}, x_{20}\right)=x_{10}+x_{20}=0
$$


Luego, el sistema (4.70)-(4.71) se reduce a,

$$
\begin{aligned}
& \frac{d \hat{z}_{1}}{d \tau}=-\hat{z}_{1}+x_{10} x_{20} \\
& \frac{d \hat{z}_{2}}{d \tau}=0,
\end{aligned}
$$

cuyos puntos de equilibrio son de la forma $\left(x_{10} x_{20}, z_{2}^{e}\right)$, para cualquier $z_{2}^{e}$, y la variedad centro correspondiente por cualquiera de estos equilibrios es $\hat{z}_{1}=x_{10} x_{20}$.

En las siguientes figuras se comparan las trayectorias del SPS y las del sistema reducido en el intervalo de tiempo $[0,4]$, para distintos valores del parámetro de perturbación $(\epsilon=, 0005,0001$ y ,00005), cuando las condiciones iniciales son,

$$
\left(x_{1}(0), x_{2}(0), z_{1}(0), z_{2}(0)\right)=(, 5,-, 5,-, 2,, 24) .
$$

Para el caso de las variables lentas sólo realizamos la comparación para $\epsilon=, 0005$, pues los gráficos para los parámetros más chicos son similares.

Para el caso de las variables rápidas $z_{1}$ y $z_{2}$, se comparan con sus correspondientes aproximaciones que se obtienen a partir de las trayectorias del sistema reducido; es decir $z_{1}$ con $z_{1 a}=\alpha_{1}\left(x_{1 r}, x_{2 r}\right)=x_{1 r} x_{2 r}$, y $z_{2}$ con $z_{2 a}=\alpha_{2}\left(x_{1 r}, x_{2 r}\right)=x_{2 r}^{2}+2 x_{1 r} x_{2 r}+x_{1 r}$. Además, para poder observar el sistema "capa límite", se grafican las trayectorias de $z_{1}$ y $z_{1 a}$ en el intervalo de tiempo $[0,, 1]$. 


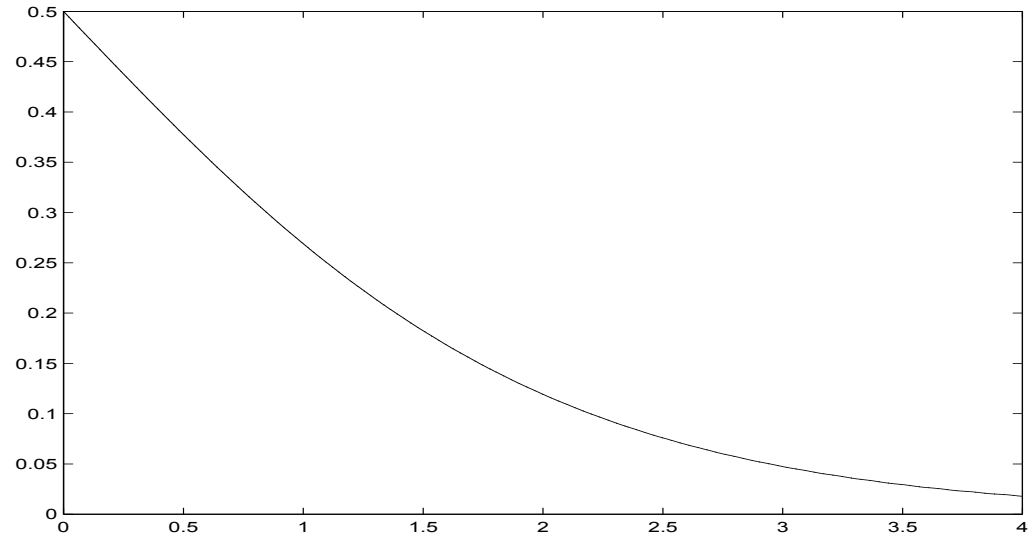

Figura 4.1: $x_{1}(t)(-)$ y $x_{1 r}(.).(t), \epsilon=, 0005$

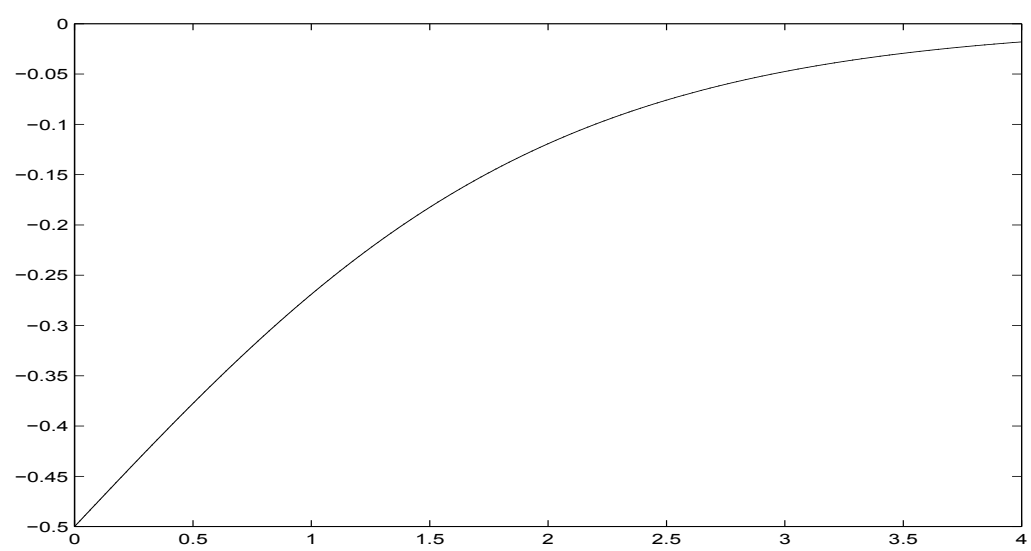

Figura 4.2: $x_{2}(t)(-)$ y $x_{2 r}(t)(.),. \epsilon=, 0005$. 


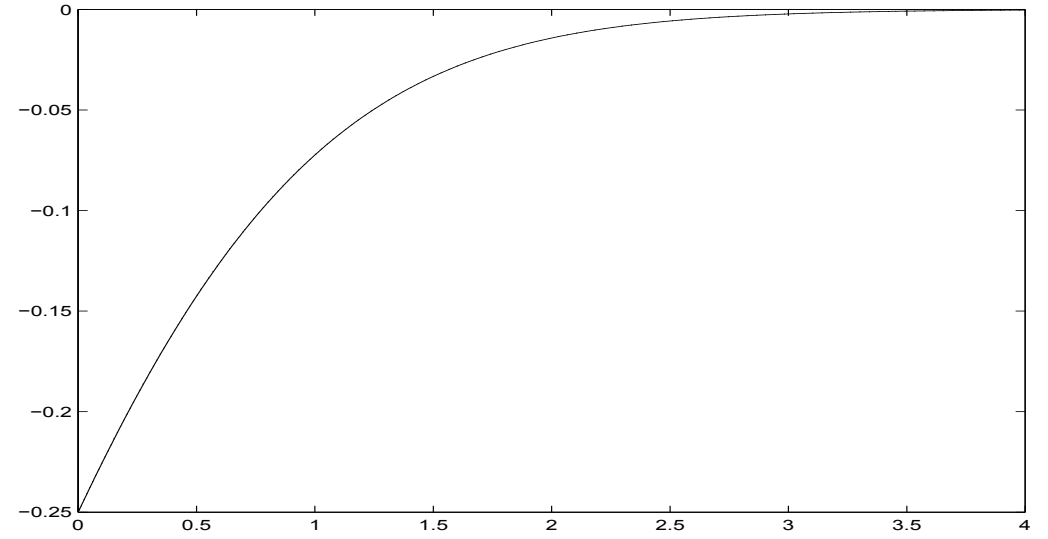

Figura 4.3: $z_{1}(t)(-)$ y $z_{1 a}(.).(t), \epsilon=, 0005$.

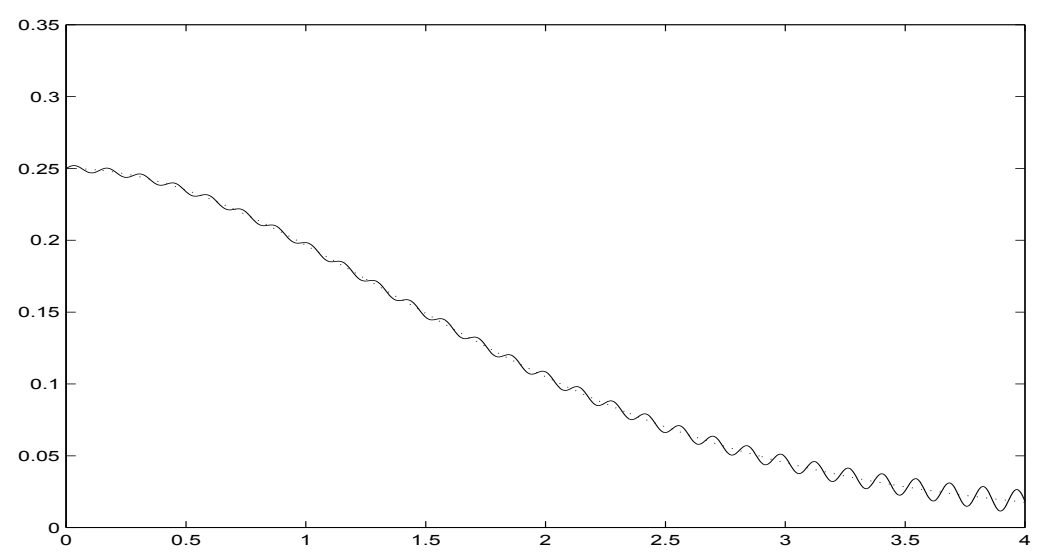

Figura 4.4: $z_{2}(t)(-)$ y $z_{2 a}(t)(.),. \epsilon=, 0005$. 


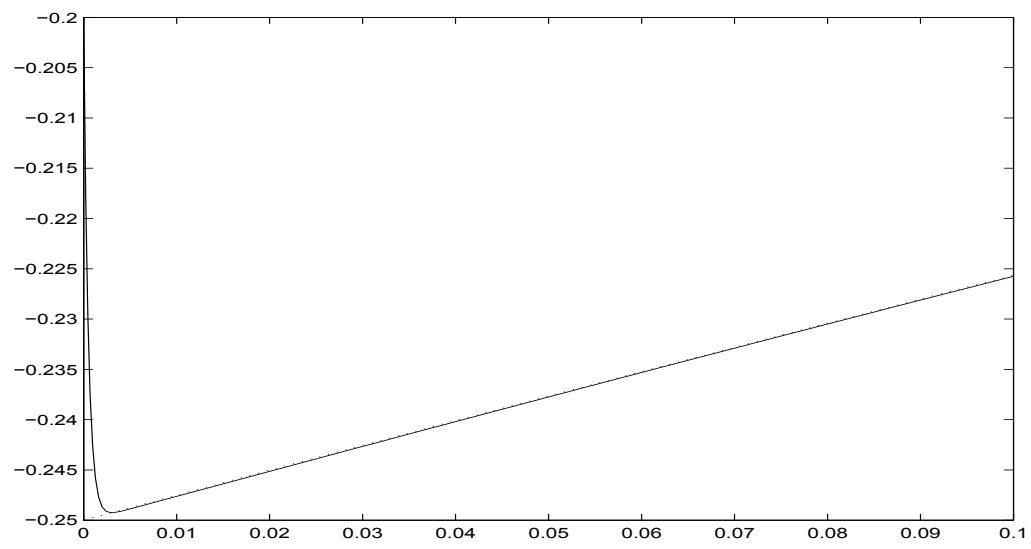

Figura 4.5: $z_{1}(t)(-)$ y $z_{1 a}(t)(.$.$) en [0,, 1], \epsilon=, 0005$.

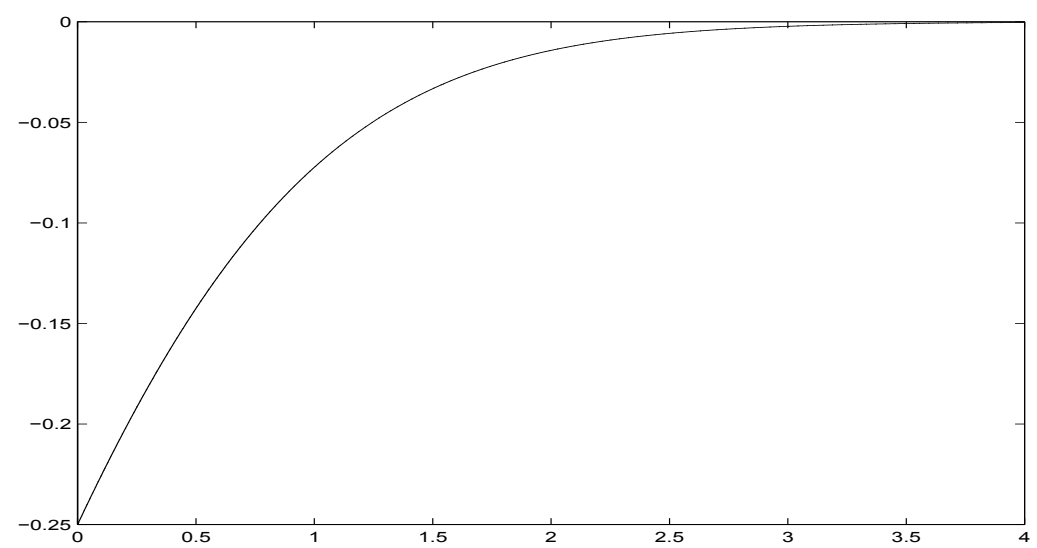

Figura 4.6: $z_{1}(t)(-)$ y $z_{1 a}(t)(.),. \epsilon=, 0001$. 


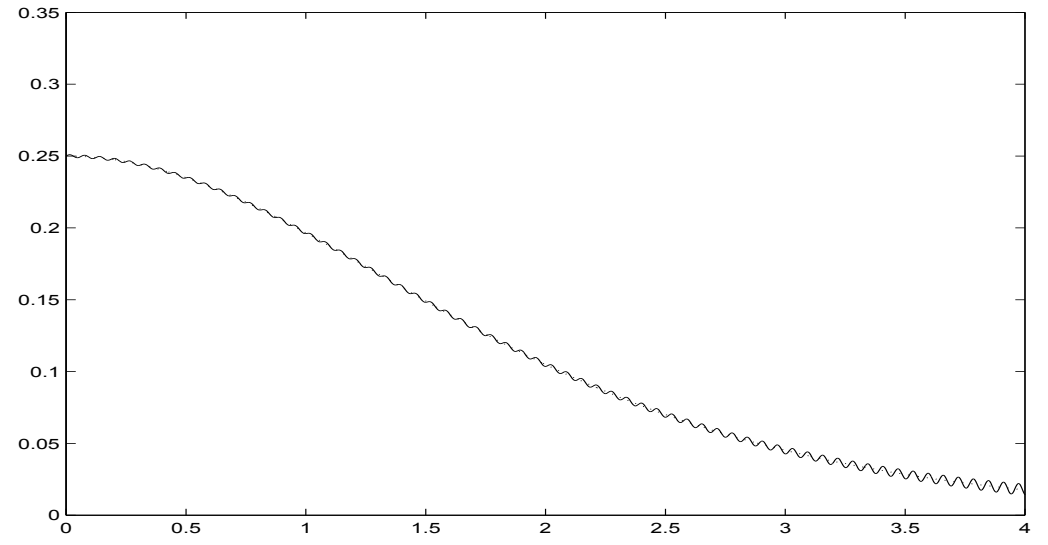

Figura 4.7: $z_{2}(t)(-)$ y $z_{2 a}(t)(.),. \epsilon=, 0001$.

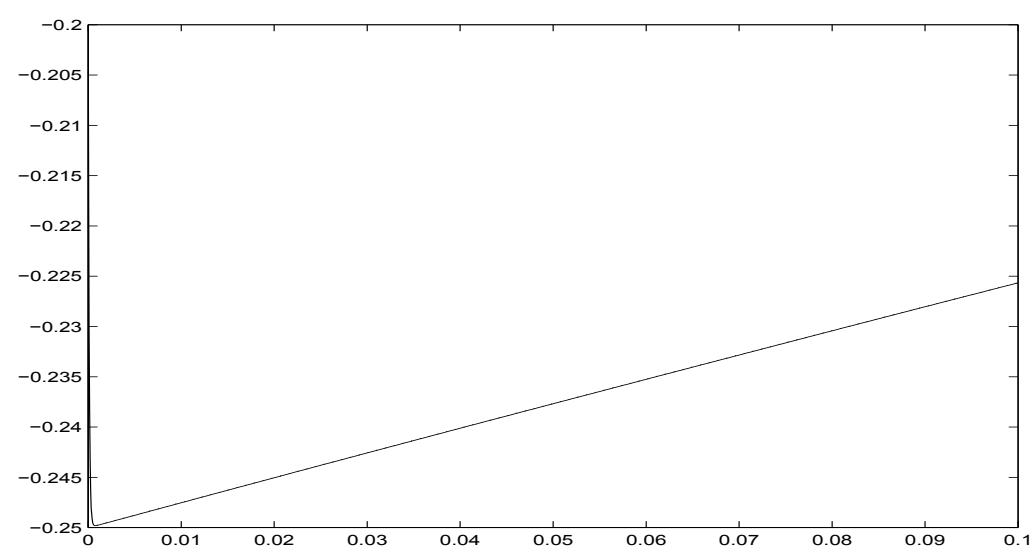

Figura 4.8: $z_{1}(t)(-)$ y $z_{1 a}(t)(.$.$) en [0,, 1], \epsilon=, 0001$. 


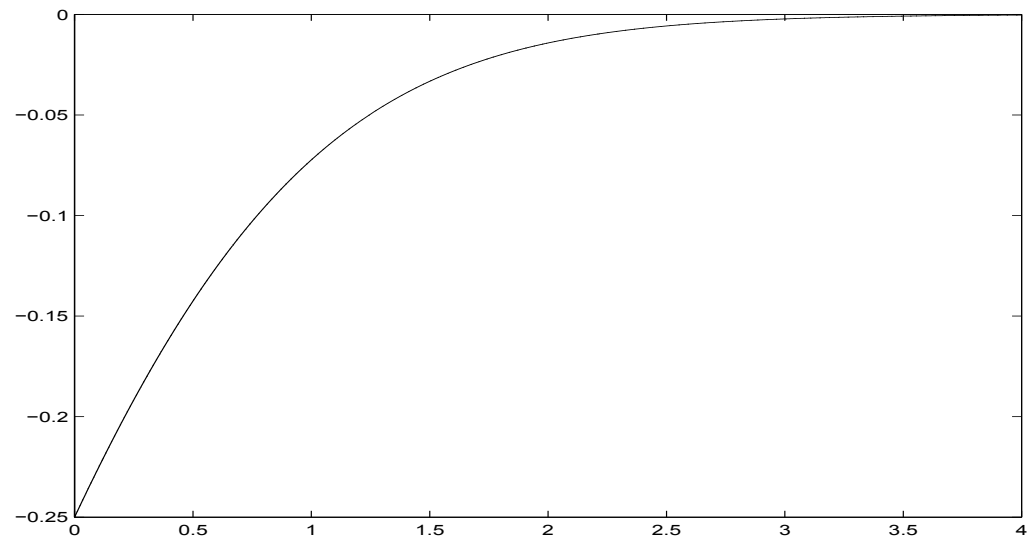

Figura 4.9: $z_{1}(t)(-)$ y $z_{1 a}(t)(.),. \epsilon=, 00005$.

Figura 4.10: $z_{2}(t)(-)$ y $z_{2 a}(t)(.),. \epsilon=, 00005$. 


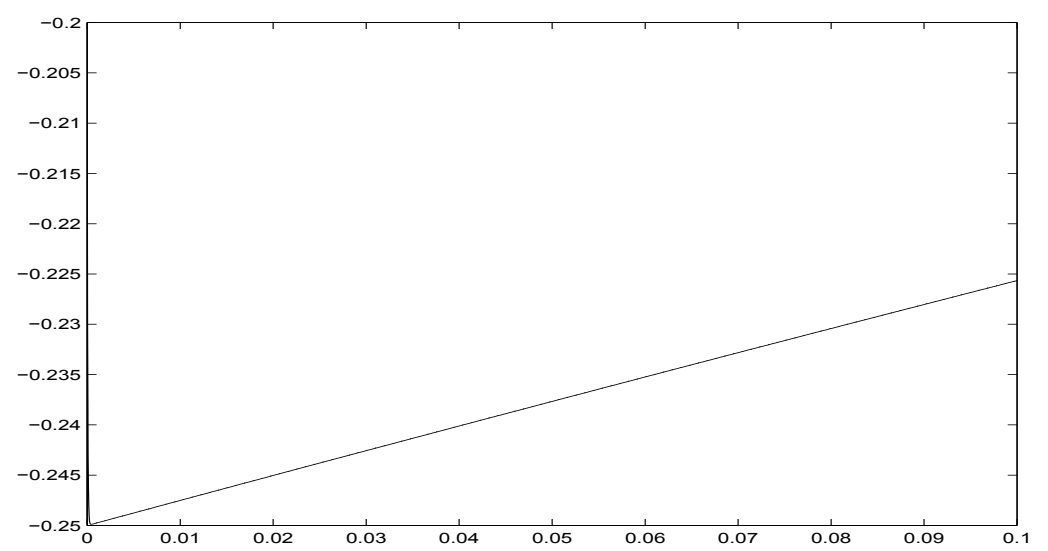

Figura 4.11: $z_{1}(t)(-)$ y $z_{1 a}(t)(.$.$) en [0,, 1], \epsilon=, 00005$. 
72 CAPÍTULO 4. SISTEMAS CON PERTURBACIÓN SINGULAR 


\section{Capítulo 5}

\section{SDAS: un problema de control}

\subsection{Introducción}

Como hemos visto brevemente en la Sección 1.5, existen diferentes tipos de problemas de aplicación donde los SDA aparecen con frecuencia.

Un problema particular es el llamado prescribed path control (PPC). En este caso se considera el modelo de un proceso (o planta) dado por:

$$
\dot{x}(t)=f(x(t), u(t), t)
$$

donde $x$ representa el vector de variables diferenciales o variables de estado del sistema y $u$ el de variables de control. El objetivo es elegir $u(t)$ adecuadamente de modo tal que la trayectoria $x(t)$ siga algún camino prefijado,

$$
g(x(t), u(t), t)=0
$$

Observar que (5.1)-(5.2) resulta un SDAS. Con frecuencia $u$ no aparece en (5.2), y luego el SDAS es de índice mayor que uno.

Dentro de los casos particulares correspondientes al problema PPC citaremos: 
1. El PPC de un brazo de robot con junta flexible [33, 34], donde pueden considerarse dos situaciones. Si existe un punto de contacto que se está moviendo a lo largo de una superficie, entonces la restricción está impuesta por la superficie. La superficie aplica fuerzas de reacción sobre el robot y el problema es con frecuencia analizado en forma variacional (aplicando el principio de las deformaciones virtuales). Si en cambio el robot se está moviendo libremente en el espacio de trabajo y la restricción (5.2) aparece para evitar una colisión con objetos fijos, entonces el problema debería modelarse considerando a (5.1) como la dinámica libre e imponiendo la restricción (5.2).

2. Problemas de trayectoria PPC: en este caso el SDA modela una nave volando en el espacio cuando las restricciones algebraicas del camino se imponen sobre sus trayectoria, [4]. En este problema las ecs. (5.1) incluyen las ecuaciones de movimiento del vehículo, mientras que una ecuación algebraica de la forma

$$
g(x(t), t)=0
$$

describe las trayectorias. El vector de variables de estado $x$ representa la posición y velocidad del vehículo. Se elige a $u$ (variable de control) como el ángulo bank.

3. Otro tipo de problemas de PPC surge cuando existen invariantes (es decir, relaciones que valen a lo largo de cualquier trayectoria solución) presentes en la solución de un SDO. Estos invariantes pueden ser igualdades (o en general desigualdades) que describen la conservación total de energía, masa o momento de un sistema. Los problemas de este tipo 
se pueden formular como SDA donde los invariantes se imponen como restricciones algebraicas, [35].

\section{Planteo del problema}

En este capítulo trabajaremos con sistemas no-lineales, afines en el control, de la forma

$$
\dot{x}=f(x)+g(x) u
$$

con $f: \mathbb{R}^{n} \rightarrow \mathbb{R}^{n}$ y $g: \mathbb{R}^{n} \rightarrow \mathbb{R}^{m}$ suficientemente suaves; $x \in \mathbb{R}^{n}$ vector de variables de estado y $u \in \mathbb{R}^{m}$ vector de variables de control. Supondremos que las soluciones de (5.4) deben satisfacer la restricción algebraica

$$
k(x)=0
$$

con $k: \mathbb{R}^{n} \rightarrow \mathbb{R}^{m}$ suave y $r g\left(\frac{\partial k}{\partial x}\right)=m$.

Las siguientes definiciones nos ayudarán a formular en forma precisa el problema que nos ocupará.

Definición 5.1: Consideremos el sistema no-lineal

$$
\dot{x}=f(x),
$$

una variedad $N \subset \mathbb{R}^{n}$ se dice invariante para (5.6) si

$$
f(x) \in T_{x} N, \quad \forall x \in N
$$

Observar que si $N$ es una variedad conexa, esto implica que las soluciones de (5.6), para $x(0) \in N$, permanecen en $N$ para todo $t \geq 0$.

Definición 5.2: Dado el sistema (5.4), una variedad $N \subset \mathbb{R}^{n}$ se llama (localmente) invariante por realimentación de estados si existe (localmente 
sobre $N$ ) una realimentación de estados estática $u=\alpha(x)$, tal que $N$ es invariante para el sistema realimentado

$$
\dot{x}=f(x)+g(x) \alpha(x)
$$

es decir,

$$
f(x)+g(x) \alpha(x) \in T_{x} N, \quad \forall x \in N .
$$

En nuestro caso, considerando la superficie suave

$$
S=\left\{x \in \mathbb{R}^{n}: k(x)=0\right\},
$$

nuestro objetivo será hallar un control por realimentación de estados $u=$ $\alpha(x)$ y la subvariedad $\mu \subset S$ maximal, invariante para el sistema realimentado. De este modo, realimentando el sistema con $u=\alpha(x)$ y considerndo $x(0) \in \mu$, nos aseguramos que las trayectorias permanecen en $\mu$.

Para resolver este problema consideraremos el sistema compuesto (5.4)-(5.5), que resulta un SDAS si tomamos a $x$ como el vector de variables diferenciales del sistema y a $u$ como el vector de las variables algebraicas.

Kumar y Daoutidis [26], analizaron el comportamiento de un SDAS con control de la forma:

$$
\begin{aligned}
\dot{x} & =f(x)+b(x) z+g(x) u \\
0 & =k(x)+l(x) z+c(x) u \\
y_{i} & =h_{i}(x), i=1, \ldots, m .
\end{aligned}
$$

donde $x \in \chi \subset \mathbb{R}^{n}$ es el vector de variables diferenciales del sistema, $z \in \Omega \subset$ $\mathbb{R}^{p}$ es el vector de variables algebraicas ( $\chi$ y $\Omega$ conjuntos conexos y abiertos), $u \in \mathbb{R}^{m}$ es el vector de variables de control e $y_{i}$ es la i-ésima salida, que se supone una función suave de las variables diferenciales. 
Suponiendo $l(x)$ singular (es decir, el SDAS es de índice mayor que uno), los autores se ocuparon de dos problemas:

1. Obtener una realización en el espacio de estados del SDAS, es decir un conjunto de ecuaciones diferenciales para $x$ que describen la dinámica del sistema bajo restricciones algebraicas.

2. Formular y resolver un problema de control a partir de la realización en el espacio de estados obtenida, utilizando resultados conocidos para SDO con control.

A su vez el paso 1 involucra:

(i) Identificar las ecuaciones algebraicas para $x$ impuestas por las ecuaciones singulares; estas restricciones dan lugar a la región del espacio de estados donde las variables diferenciales evolucionan.

(ii) Obtener una solución para las variables algebraicas $z$ en términos de $x$ y $u$, consistente con las restricciones algebraicas ya identificadas para $x$.

En nuestro caso aplicaremos el algoritmo propuesto por los autores para resolver el problema 1, pero para el caso particular del SDAS (5.4)-(5.5). Consideraremos a las variables de control $u$ como variables algebraicas, lo que nos permitirá hallar una realimentación $u=\alpha(x)$ y luego una realización del SDAS en el espacio de estados (que en este caso corresponde al sistema realimentado) junto con un conjunto de restricciones algebraicas (que dependerán sólo de las variables diferenciales).

Por último, aplicaremos la técnica desarrollada para hallar la dinámica cero de un sistema no-lineal de múltiples entradas y múltiples salidas sin grado relativo vectorial, [36]. 


\subsection{Caso $m=1$}

En esta Sección consideraremos el SDAS (5.4)-(5.5) para el caso particular de $u \in \mathbb{R}$ y $k: \mathbb{R}^{n} \rightarrow \mathbb{R}$. Definimos $L_{f} k(x)=\frac{d k}{d x} f(x)$ y recursivamente $L_{f}^{r} k(x)=L_{f}\left(L_{f}^{r-1} k(x)\right)$.

\section{Caso I:}

$$
L_{g} k(x) \neq 0
$$

Diferenciando la restricción algebraica $k(x)=0$ respecto de $t$ se tiene,

$$
\frac{\partial k}{\partial x}(x)(f(x)+g(x) u(t))=L_{f} k(x)+L_{g} k(x) u(t)=0
$$

y se obtiene la realimentación de estados:

$$
u_{1}=\alpha_{1}(x)=-\frac{L_{f} k(x)}{L_{g} k(x)} .
$$

Observar que si la condición (5.11) se cumple en un punto $x^{0}$, entonces la realimentación $u_{1}$ estará definida localmente alrededor de $x^{0}$. Se concluye entonces,

Proposición 5.1: Supongamos (5.11), entonces el sistema (5.4)-(5.5) realimentado con $u_{1}=\alpha_{1}(x)$ es un SDA de índice 1 y espacio de configuración $S$. Luego, en este caso $S$ es la variedad invariante maximal por realimentación de estados. 


\section{Demostración:}

Consideremos el sistema (5.4)-(5.5) realimentado con $u_{1}=\alpha_{1}(x)$, se obtiene el siguiente SDO con restricciones algebraicas (del tipo (3.15)-(3.16)),

$$
\begin{aligned}
\dot{x} & =f(x)-g(x) \frac{L_{f} k(x)}{L_{g} k(x)} \\
0 & =k(x) .
\end{aligned}
$$

Diferenciando la restricción algebraica respecto del tiempo se tiene,

$$
\frac{\partial k}{\partial x} \dot{x}=\frac{\partial k}{\partial x}\left(f(x)-\frac{L_{f} k(x)}{L_{g} k(x)} g(x)\right)=0,
$$

entonces, (5.12)-(5.13) es un SDA de índice 1 y espacio de configuración $S$. Luego, la variedad invariante maximal por realimentación de estados es en este caso $S$.

\section{Caso II:}

$$
L_{g} k(x)=0 \text { y } L_{g} L_{f} k(x) \neq 0
$$

Diferenciando la restricción $k(x)=0$ respecto de $t$ se obtiene,

$$
L_{f} k(x)=0,
$$

y diferenciando nuevamente esta expresión,

$$
L_{f}^{2} k(x)+L_{g} L_{f} k(x) u=0 .
$$

De la igualdad anterior resulta la realimentación:

$$
u_{2}=\alpha_{2}(x)=-\frac{L_{f}^{2} k(x)}{L_{g} L_{f} k(x)} .
$$


Proposición 5.2: Si se cumple (5.14), el sistema (5.4)-(5.5) realimentado con $u_{2}=\alpha_{2}(x)$ tiene índice 2 y espacio de configuración

$$
\mu_{2}=\left\{x \in \mathbb{R}^{n}: k(x)=0, L_{f} k(x)=0\right\} .
$$

Demostración:

El sistema (5.4)-(5.5) realimentado es,

$$
\begin{aligned}
\dot{x} & =f(x)-g(x) \frac{L_{f}^{2} k(x)}{L_{g} L_{f} k(x)} \\
0 & =k(x)
\end{aligned}
$$

Diferenciando $k(x)=0$ y $L_{f} k(x)=0$ respecto del tiempo se obtienen, respectivamente,

$$
\frac{\partial k}{\partial x}(x)\left(f(x)-\frac{L_{f}^{2} k(x)}{L_{g} L_{f} k(x)} g(x)\right)=L_{f} k(x)-\frac{L_{f}^{2} k(x)}{L_{g} L_{f} k(x)} L_{g} k(x)=L_{f} k(x)=0
$$

y

$$
\frac{\partial\left(L_{f} k(x)\right)}{\partial x}\left(f(x)-\frac{L_{f}^{2} k(x)}{L_{g} L_{f} k(x)} g(x)\right)=L_{f}^{2} k(x)-\frac{L_{f}^{2} k(x)}{L_{g} L_{f} k(x)} L_{g} L_{f} k(x)=0 .
$$

Luego, el sistema realimentado tiene índice 2 y espacio de configuración $\mu_{2}$. Observar que por ser $\mu_{2}$ el espacio de configuración del sistema realimentado, resulta maximal (consecuencia de la definición de espacio de configuración dada en la Sección 3.1).

Generalizando, supongamos que,

$$
L_{g} L_{f}^{r} k(x)=0, \quad \text { si } r<s-1 \quad \text { y } \quad L_{g} L_{f}^{s-1} k(x) \neq 0
$$


Diferenciando sucesivamente la restricción $k(x)=0$ se tiene,

$$
\begin{aligned}
L_{f} k(x)+L_{g} k(x) u & =L_{f} k(x)=0 \\
L_{f}^{2} k(x)+L_{g} L_{f} k(x) u & =L_{f}^{2} k(x)=0 \\
& \cdots \\
L_{f}^{s-1} k(x)+L_{g} L_{f}^{s-2} k(x) u & =L_{f}^{s-1} k(x)=0 \\
L_{f}^{s} k(x)+L_{g} L_{f}^{s-1} k(x) u & =0
\end{aligned}
$$

De la última igualdad se obtiene,

$$
u_{s}=\alpha_{s}(x)=-\frac{L_{f}^{s} k(x)}{L_{g} L_{f}^{s-1} k(x)},
$$

y concluimos con el siguiente resultado,

Proposición 5.3: Asumiendo (5.17), entonces el SDAS (5.4)-(5.5) realimentado con $u_{s}=\alpha_{s}(x)$ tiene índice $s$ y espacio de configuración

$$
\mu_{s}=\left\{x \in \mathbb{R}^{n}: k(x)=0, L_{f} k(x)=0, \ldots, L_{f}^{s-1} k(x)=0\right\}
$$

\section{Demostración:}

El sistema (5.4)-(5.5) realimentado con $u_{s}=\alpha_{s}(x)$ es,

$$
\begin{aligned}
\dot{x} & =f(x)-g(x) \frac{L_{f}^{s} k(x)}{L_{g} L_{f}^{s-1} k(x)} \\
0 & =k(x),
\end{aligned}
$$

luego, si $x \in \mu_{s}$, se tiene

$$
\begin{aligned}
\frac{\partial k}{\partial x} \dot{x} & =L_{f} k(x)=0 \\
\frac{\partial\left(L_{f} k\right)}{\partial x} \dot{x} & =L_{f}^{2} k(x)=0 \\
& \cdots \\
\frac{\partial\left(L_{f}^{s-2} k\right)}{\partial x} \dot{x} & =L_{f}^{s-1} k(x)=0 \\
\frac{\partial\left(L_{f}^{s-1} k\right)}{\partial x} \dot{x} & =L_{f}^{s} k(x)-\frac{L_{f}^{s} k(x)}{L_{g} L_{f}^{s-1} k(x)} L_{g} L_{f}^{s-1} k(x)=0,
\end{aligned}
$$

Nuevamente observamos que la variedad obtenida es maximal pues es el espacio de configuración del sistema realimentado. $\square$ 
La proposición anterior generaliza el problema cualquiera sea $s$. Por ejemplo, si $s=1$, resulta la Proposición 5.1, mientras que para $s=2$ se obtiene la Proposición 5.2.

\subsection{Caso $m>1$}

En esta sección analizaremos el problema planteado en la Introducción para el caso en que el sistema (5.4) tenga $m$ controles $(m>1)$. Supondremos $g(x)=\left[\begin{array}{llll}g_{1}(x) & \ldots & g_{m}(x)\end{array}\right]^{\prime}$ y $k(x)=\left[\begin{array}{llll}k_{1}(x) & \ldots & k_{m}(x)\end{array}\right]^{\prime}$. Las condiciones de rango constante que se establecen a lo largo del algoritmo son locales, es decir valen en un entorno de un punto de operación.

- Paso 1:

Renombramos $k^{1}(x)=k(x)$; y diferenciamos $k^{1}(x)=0$ con respecto a $t$,

$$
\frac{\partial k^{1}}{\partial x}(f(x)+g(x) u)=L_{f} k^{1}(x)+L_{g} k^{1}(x) u=0,
$$

siendo

$$
L_{f} k^{1}(x)=\left[\begin{array}{c}
L_{f} k_{1}^{1}(x) \\
\vdots \\
L_{f} k_{m}^{1}(x)
\end{array}\right], \quad L_{g} k^{1}(x)=\left[\begin{array}{ccc}
L_{g_{1}} k_{1}^{1}(x) & \ldots & L_{g_{m}} k_{1}^{1}(x) \\
\vdots & & \vdots \\
L_{g_{1}} k_{m}^{1}(x) & \ldots & L_{g_{m}} k_{m}^{1}(x)
\end{array}\right]
$$

Evaluamos,

$$
\operatorname{rg}\left(L_{g} k^{1}(x)\right)=m_{1}
$$

\section{Caso I:}

$$
m_{1}=m
$$

En este caso el algoritmo termina y se obtiene

$$
u_{1}=\alpha_{1}(x)=-\left(L_{g} k^{1}(x)\right)^{-1} L_{f} k^{1}(x) .
$$


Se concluye con el siguiente resultado,

Proposición 5.4: Supongamos que $r g\left(L_{g} k^{1}(x)\right)=m$, entonces el sistema (5.4)-(5.5) realimentado con (5.20) tiene índice 1. Además la variedad

$$
\mu_{1}=\left\{x \in \mathbb{R}^{n}: k^{1}(x)=0\right\}
$$

es la variedad invariante maximal para el sistema (5.4) realimentado con (5.20).

Demostración: análoga a la prueba de la Proposición 5.1.

\section{Caso II:}

$$
m_{1}<m
$$

Entonces, existe una matriz no singular y suave $E_{1}(x)$ de dimensión $m \times m$ tal que,

(i) Las primeras $m_{1}$ filas en $E_{1}(x) L_{g} k^{1}(x)$ resultan linealmente independientes $\mathrm{y}$,

(ii) las últimas $m-m_{1}$ filas se anulan.

Premultiplicando la restricción $L_{f} k^{1}(x)+L_{g} k^{1}(x) u=0$ por $E_{1}(x)$ se obtiene:

$$
0=\left[\begin{array}{l}
\bar{k}^{2}(x) \\
k^{2}(x)
\end{array}\right]+\left[\begin{array}{c}
\bar{l}^{2}(x) \\
0
\end{array}\right] u
$$

$\operatorname{con} r g \bar{l}^{2}=m_{1}$

- Paso 2:

Diferenciando la restricción $k^{2}(x)=0$ respecto de $t$ se obtiene,

$$
\frac{\partial k^{2}}{\partial x}(f(x)+g(x) u)=L_{f} k^{2}(x)+L_{g} k^{2}(x) u=0 .
$$


Se evalúa

$$
r g\left[\begin{array}{l}
\bar{l}^{2}(x) \\
L_{g} k^{2}(x)
\end{array}\right]=m_{2}
$$

\section{Caso I:}

$$
m_{2}=m
$$

En este caso el algoritmo termina y de las expresiones:

$$
\begin{aligned}
\bar{k}^{2}(x)+\bar{l}^{2}(x) u & =0 \\
L_{f} k^{2}(x)+L_{g} k^{2}(x) u & =0,
\end{aligned}
$$

se obtiene

$$
u_{2}=\alpha_{2}(x)=-\left[\begin{array}{l}
\bar{l}^{2}(x) \\
L_{g} k^{2}(x)
\end{array}\right]^{-1}\left[\begin{array}{l}
\bar{k}^{2}(x) \\
L_{f} k^{2}(x)
\end{array}\right] .
$$

Se tiene entonces que,

Proposición 5.5: Si $r g\left(L_{g} k^{1}(x)\right)=m_{1}<m$ y $r g\left[\bar{l}^{2}(x) L_{g} k^{2}(x)\right]^{\prime}=m$, entonces el sistema (5.4)-(5.5) realimentado con (5.23) tiene índice 2 y espacio de configuración $\mu_{2}=\left\{x \in \mathbb{R}^{n}: k^{1}(x)=0, k^{2}(x)=0\right\}$.

\section{Demostración:}

Consideremos el sistema (5.4)-(5.5) realimentado con (5.23),

$$
\begin{aligned}
\dot{x} & =f(x)-g(x)\left[\begin{array}{l}
\bar{l}^{2}(x) \\
L_{g} k^{2}(x)
\end{array}\right]^{-1}\left[\begin{array}{l}
\bar{k}^{2}(x) \\
L_{f} k^{2}(x)
\end{array}\right] \\
0 & =k^{1}(x) .
\end{aligned}
$$

Diferenciando $k^{1}(x)=0$ se tiene,

$$
\begin{aligned}
& \frac{\partial k^{1}}{\partial x}\left(f(x)-g(x)\left[\begin{array}{l}
\bar{l}^{2}(x) \\
L_{g} k^{2}(x)
\end{array}\right]^{-1}\left[\begin{array}{c}
\bar{k}^{2}(x) \\
L_{f} k^{2}(x)
\end{array}\right]\right)= \\
& L_{f} k^{1}(x)-L_{g} k_{1}(x)\left[\begin{array}{l}
\bar{l}^{2}(x) \\
L_{g} k^{2}(x)
\end{array}\right]^{-1}\left[\begin{array}{c}
\bar{k}^{2}(x) \\
L_{f} k^{2}(x)
\end{array}\right],
\end{aligned}
$$


y premultiplicando por $E_{1}(x)$ se obtiene,

$$
\left[\begin{array}{l}
\bar{k}^{2}(x) \\
k^{2}(x)
\end{array}\right]-\left[\begin{array}{c}
\bar{l}^{2}(x) \\
0
\end{array}\right]\left[\begin{array}{l}
\bar{l}^{2}(x) \\
L_{g} k^{2}(x)
\end{array}\right]^{-1}\left[\begin{array}{l}
\bar{k}^{2}(x) \\
L_{f} k^{2}(x)
\end{array}\right] .
$$

Por otro lado si consideramos las restricción $k^{2}(x)=0$ y derivamos respecto del tiempo obtenemos,

$$
\begin{gathered}
\frac{\partial k^{2}}{\partial x}\left(f(x)-g(x)\left[\begin{array}{l}
\bar{l}^{2}(x) \\
L_{g} k^{2}(x)
\end{array}\right]^{-1}\left[\begin{array}{l}
\bar{k}^{2}(x) \\
L_{f} k^{2}(x)
\end{array}\right]\right)= \\
L_{f} k^{2}(x)-L_{g} k^{2}(x)\left[\begin{array}{c}
\bar{l}^{2}(x) \\
L_{g} k^{2}(x)
\end{array}\right]^{-1}\left[\begin{array}{c}
\bar{k}^{2}(x) \\
L_{f} k^{2}(x)
\end{array}\right] .
\end{gathered}
$$

Por último, de lo que resulta de diferenciar ambas restricciones se tiene:

$$
\left[\begin{array}{c}
\bar{k}^{2}(x) \\
L_{f} k^{2}(x)
\end{array}\right]-\left[\begin{array}{c}
\bar{l}^{2}(x) \\
L_{g} k^{2}(x)
\end{array}\right]\left[\begin{array}{l}
\bar{l}^{2}(x) \\
L_{g} k^{2}(x)
\end{array}\right]^{-1}\left[\begin{array}{l}
\bar{k}^{2}(x) \\
L_{f} k^{2}(x)
\end{array}\right]=0 .
$$

Luego, el sistema realimentado tiene índice 2 y espacio de configuración $\mu_{2}$. $\square$

\section{Caso II:}

$$
m_{2}<m
$$

Entonces, existe una matriz $E_{2}(x)$ no singular y suave de dimensión $m \times m$ tal que,

(i) Las primeras $m_{2}$ filas en $E_{2}(x)\left[\bar{l}^{2}(x) \quad L_{g} k^{2}(x)\right]^{\prime}$ resultan linealmente independientes $\mathrm{y}$,

(ii) las últimas $m-m_{2}$ filas se anulan.

Luego,

$$
E_{2}(x)\left\{\left[\begin{array}{c}
\bar{k}^{2}(x) \\
L_{f} k^{2}(x)
\end{array}\right]+\left[\begin{array}{c}
\bar{l}^{2}(x) \\
L_{g} k^{2}(x)
\end{array}\right] u\right\}=\left[\begin{array}{c}
\bar{k}^{3}(x) \\
k^{3}(x)
\end{array}\right]+\left[\begin{array}{c}
\bar{l}^{3}(x) \\
0
\end{array}\right] u,
$$

$\operatorname{con} r g \bar{l}^{3}(x)=m_{2}$. 
- Paso 3:

Derivamos la restricción $k^{3}(x)=0$,

$$
L_{f} k^{3}(x)+L_{g} k^{3}(x) u=0
$$

y evaluamos

$$
r g\left[\begin{array}{c}
\bar{l}^{3}(x) \\
L_{g} k^{3}(x)
\end{array}\right]=m_{3}
$$

\section{Caso I:}

$$
m_{3}=m
$$

Si esto ocurre el algoritmo termina y de

$$
\begin{aligned}
\bar{k}^{3}(x)+\bar{l}^{3}(x) u & =0 \\
L_{f} k^{3}(x)+L_{g} k^{3}(x) u & =0,
\end{aligned}
$$

se obtiene

$$
u_{3}=\alpha_{3}(x)=-\left[\begin{array}{c}
\bar{l}^{3}(x) \\
L_{g} k^{3}(x)
\end{array}\right]^{-1}\left[\begin{array}{c}
\bar{k}^{3}(x) \\
L_{f} k^{3}(x)
\end{array}\right] .
$$

Luego, se concluye:

Proposición 5.6: Supongamos que

$$
\begin{aligned}
r g\left(L_{g} k^{1}(x)\right)= & m_{1}<m, \operatorname{rg}\left[\bar{l}^{2}(x) L_{g} k^{2}(x)\right]^{\prime}=m_{2}<m \\
& r g\left[\bar{l}^{3}(x) L_{g} k^{3}(x)\right]^{\prime}=m
\end{aligned}
$$

entonces el sistema (5.4)-(5.5) realimentado con (5.28) tiene índice 3 y espacio de configuración

$$
\mu_{3}=\left\{x \in \mathbb{R}^{n}: k^{1}(x)=0, k^{2}(x)=0, k^{3}(x)=0\right\} .
$$




\section{Demostración:}

El sistema (5.4)-(5.5) realimentado con (5.28) es:

$$
\begin{aligned}
\dot{x} & =f(x)-g(x)\left(\left[\begin{array}{c}
\bar{l}^{3}(x) \\
L_{g} k^{3}(x)
\end{array}\right]^{-1}\left[\begin{array}{c}
\bar{k}^{3}(x) \\
L_{f} k^{3}(x)
\end{array}\right]\right) \\
0 & =k^{1}(x)
\end{aligned}
$$

Diferenciando las restricciones $k^{1}(x)=0$ y $k^{2}(x)=0$, y premultiplicando por $E_{1}(x)$ y $E_{2}(x)$, respectivamente, se tiene:

$$
\left[\begin{array}{c}
\bar{k}^{2}(x) \\
k^{2}(x)
\end{array}\right]-\left[\begin{array}{c}
\bar{l}^{2}(x) \\
0
\end{array}\right]\left(\left[\begin{array}{c}
\bar{l}^{3}(x) \\
L_{g} k^{3}(x)
\end{array}\right]^{-1}\left[\begin{array}{c}
\bar{k}^{3}(x) \\
L_{f} k^{3}(x)
\end{array}\right]\right)=0
$$

y

$$
\left[\begin{array}{c}
\bar{k}^{3}(x) \\
k^{3}(x)
\end{array}\right]-\left[\begin{array}{c}
\bar{l}^{3}(x) \\
0
\end{array}\right]\left(\left[\begin{array}{c}
\bar{l}^{3}(x) \\
L_{g} k^{3}(x)
\end{array}\right]^{-1}\left[\begin{array}{c}
\bar{k}^{3}(x) \\
L_{f} k^{3}(x)
\end{array}\right]\right)=0 .
$$

Luego, derivamos la restricción $k^{3}(x)=0$,

$$
L_{f} k^{3}(x)-L_{g} k^{3}(x)\left(\left[\begin{array}{c}
\bar{l}^{3}(x) \\
L_{g} k^{3}(x)
\end{array}\right]^{-1}\left[\begin{array}{c}
\bar{k}^{3}(x) \\
L_{f} k^{3}(x)
\end{array}\right]\right)=0 .
$$

Por último obtenemos,

$$
\left[\begin{array}{c}
\bar{k}^{3}(x) \\
L_{f} k^{3}(x)
\end{array}\right]-\left[\begin{array}{c}
\bar{l}^{3}(x) \\
L_{g} k^{3}(x)
\end{array}\right]\left(\left[\begin{array}{c}
\bar{l}^{3}(x) \\
L_{g} k^{3}(x)
\end{array}\right]^{-1}\left[\begin{array}{c}
\bar{k}^{3}(x) \\
L_{f} k^{3}(x)
\end{array}\right]\right)=0 ;
$$

lo que concluye la demostración.

Generalizando, supongamos que

$$
r g\left[\bar{l}^{s-1}(x) L_{g} k^{s-1}(x)\right]^{\prime}=m_{s-1}<m ;
$$

entonces existe $E_{s-1}(x)$ no singular y suave de dimensión $m \times m$ tal que,

$$
\begin{aligned}
& E_{s-1}(x)\left(\left[\begin{array}{c}
\bar{k}^{s-1}(x) \\
L_{f} k^{s-1}(x)
\end{array}\right]+\left[\begin{array}{c}
\bar{l}^{s-1}(x) \\
L_{g} k^{s-1}(x)
\end{array}\right] u\right)=\left[\begin{array}{c}
\bar{k}^{s}(x) \\
k^{s}(x)
\end{array}\right]+\left[\begin{array}{c}
\bar{l}^{s}(x) \\
0
\end{array}\right] u=0 . \\
& \operatorname{con} r g \bar{l}^{s}(x)=m_{s-1} .
\end{aligned}
$$


- Paso s:

Diferenciamos $k^{s}(x)$ respecto de $t$,

$$
\frac{\partial k^{s}}{\partial x}(f(x)+g(x) u)=0
$$

y suponemos que

$$
r g\left[\begin{array}{c}
\bar{l}^{s}(x) \\
L_{g} k^{s}(x)
\end{array}\right]=m
$$

Luego, de la expresión

$$
\left[\begin{array}{c}
\bar{k}^{s}(x) \\
L_{f} k^{s}(x)
\end{array}\right]+\left[\begin{array}{c}
\bar{l}^{s}(x) \\
L_{g} k^{s}(x)
\end{array}\right] u=\left[\begin{array}{l}
0 \\
0
\end{array}\right]
$$

obtenemos

$$
u_{s}=\alpha_{s}(x)=-\left[\begin{array}{c}
\bar{l}^{s}(x) \\
L_{g} k^{s}(x)
\end{array}\right]^{-1}\left[\begin{array}{c}
\bar{k}^{s}(x) \\
L_{f} k^{s}(x)
\end{array}\right]
$$

Concluimos esta Sección enunciando el siguiente resultado que generaliza los obtenidos para los casos de índice 1, 2 y 3.

Proposición 5.7: Supongamos que,

$$
\begin{aligned}
& r g\left[L_{g} k^{1}(x)\right]=m_{1}<m, \operatorname{rg}\left[\begin{array}{ll}
l^{2}(x) & L_{g} k^{2}(x)
\end{array}\right]^{\prime}=m_{2}<m, \ldots, \\
& r g\left[\begin{array}{lll}
\bar{l}^{s-1} & \left.L_{g} k^{s-1}(x)\right]^{\prime}=m_{s-1}<m \text { y } r g\left[\bar{l}^{s}(x)\right. & L_{g} k^{s}(x)
\end{array}\right]^{\prime}=m .
\end{aligned}
$$

Entonces, (5.4)-(5.5) realimentado con (5.31) tiene índice $s$ y espacio de configuración

$$
\mu_{s}=\left\{x \in \mathbb{R}^{n}: k^{1}(x)=0, k^{2}(x)=0, \ldots, k^{s}(x)=0\right\} .
$$




\section{Ejemplo}

Consideremos el siguiente sistema no lineal con controles $u_{1}$ y $u_{2}$ :

$$
\begin{aligned}
& \dot{x}_{1}=x_{1} x_{4}+u_{1}+u_{2} \\
& \dot{x}_{2}=x_{3} \\
& \dot{x}_{3}=x_{4} u_{1} \\
& \dot{x}_{4}=x_{1} x_{4}+x_{2}+u_{2}
\end{aligned}
$$

junto con las restricciones algebraicas,

$$
\begin{aligned}
& x_{1}=0 \\
& x_{2}=0
\end{aligned}
$$

Queremos hallar, para el sistema (5.43)-(5.46), la variedad invariante maximal por realimentación de estados contenida en

$$
\mu_{1}=\left\{x=\left(x_{1}, x_{2}, x_{3}, x_{3}\right) \in \mathbb{R}^{4}: x_{1}=0, x_{2}=0\right\},
$$

Llamamos,

$$
k^{1}(x)=\left[\begin{array}{l}
x_{1} \\
x_{2}
\end{array}\right], f(x)=\left[\begin{array}{c}
x_{1} x_{4} \\
x_{3} \\
0 \\
x_{1} x_{4}+x_{2}
\end{array}\right], g(x)=\left[\begin{array}{cc}
1 & 1 \\
0 & 0 \\
x_{4} & 0 \\
0 & 1
\end{array}\right] .
$$

Se tiene,

$$
L_{g} k^{1}(x)=\frac{\partial k^{1}}{\partial x} g(x)=\left[\begin{array}{cc}
1 & 1 \\
0 & 0
\end{array}\right], \quad L_{f} k^{1}(x)=\left[\begin{array}{c}
x_{1} x_{4} \\
x_{3}
\end{array}\right] .
$$

Luego, $r g L_{g} k^{1}(x)=1$, y por ser su segunda fila nula, tomaremos $E_{1}(x)$ igual a la matriz identidad de orden 2 .

Entonces, en este caso llamamos,

$$
\bar{k}^{2}(x)=x_{1} x_{4}, \quad k^{2}(x)=x_{3}, \quad \bar{l}^{2}(x)=\left[\begin{array}{ll}
1 & 1
\end{array}\right]^{\prime} ;
$$


y obtenemos,

$$
L_{g} k^{2}(x)=\left[\begin{array}{ll}
x_{4} & 0
\end{array}\right], L_{f} k^{2}(x)=0
$$

y

$$
r g\left[\begin{array}{c}
\bar{l}^{2}(x) \\
L_{g} k^{2}(x)
\end{array}\right]=r g\left[\begin{array}{cc}
1 & 1 \\
x_{4} & 0
\end{array}\right]=2, \text { si } x_{4} \neq 0 .
$$

De la expresión

$$
\left[\begin{array}{c}
\bar{k}^{2}(x) \\
L_{f} k^{2}(x)
\end{array}\right]+\left[\begin{array}{c}
\bar{l}^{2}(x) \\
L_{g} k^{2}(x)
\end{array}\right]\left[\begin{array}{l}
u_{1} \\
u_{2}
\end{array}\right]=\left[\begin{array}{l}
0 \\
0
\end{array}\right]
$$

se obtiene la realimentación

$$
\left[\begin{array}{l}
u_{1} \\
u_{2}
\end{array}\right]=-\left[\begin{array}{cc}
1 & 1 \\
x_{4} & 0
\end{array}\right]^{-1}\left[\begin{array}{c}
x_{1} x_{4} \\
0
\end{array}\right]=-\left[\begin{array}{c}
0 \\
-x_{1} x_{4}
\end{array}\right]
$$

De lo anterior se tiene que el sistema (5.43)-(5.46) con las restricciones algebraicas (5.36)-(5.37) satisface las hipótesis de la Proposición 5.5. Entonces, concluimos así:

(i) El SDAS (5.43)-(5.37) realimentado con (5.38) tiene índice 2 y espacio de configuración

$$
\mu_{2}=\left\{\left(x_{1}, x_{2}, x_{3}, x_{4}\right) \in \mathbb{R}^{4}: x_{1}=0, x_{2}=0, x_{3}=0\right\} .
$$

(ii) $\mu_{2}$ es la variedad invariante maximal por realimentación de estados contenida en $\mu_{1}$.

El sistema (5.43)-(5.46) realimentado con (5.38) es,

$$
\begin{aligned}
& \dot{x}_{1}=0 \\
& \dot{x}_{2}=x_{3} \\
& \dot{x}_{3}=0 \\
& \dot{x}_{4}=x_{2}
\end{aligned}
$$


Si tomamos como condiciones iniciales $(0,0,1,1) \in \mu_{1}$, las soluciones son

$$
\begin{aligned}
& x_{1}(t)=0 \\
& x_{2}(t)=t \\
& x_{3}(t)=1 \\
& x_{4}(t)=\frac{1}{2} t^{2}+1
\end{aligned}
$$

y entonces las trayectorias no evolucionan sobre $\mu_{1}$. Por último, observar que en este caso $\mu_{2}$ es un subespacio de equilibrio para el sistema realimentado; entonces, para cualquier condición inicial en $\mu_{2}$ el sistema permanece en esa condición inicial, y luego en $\mu_{2}$.

\subsection{Dinámica cero sin grado relativo vectorial}

En esta Sección aplicaremos el algoritmo desarrollado en la sección anterior para hallar la dinámica cero de un sistema no-lineal con múltiples entradas y salidas, aún cuando ciertas hipótesis standard (como pedir que el sistema tenga grado relativo vectorial) no se verifiquen. Más adelante describiremos en forma precisa esta idea.

Trabajaremos con un sistema de la forma,

$$
\begin{aligned}
\dot{x} & =f(x)+g(x) u \\
y & =h(x)
\end{aligned}
$$

con $u \in \mathbb{R}^{m}$ y $h: \mathbb{R}^{n} \rightarrow \mathbb{R}^{m}$.

Definición: Sea $U$ un conjunto abierto de $\mathbb{R}^{n}$ y $x_{0} \in U$. Una subvariedad anuladora de la salida para el sistema (5.39)-(5.40) es una variedad conexa $M \subset U$ tal que: 
1. $x_{0} \in M$;

2. para cada $x \in M, h(x)=0$;

3. $M$ es localmente invariante por realimentación.

Entonces, si $M$ es subvariedad invariante anuladora de la salida, existe una realimentación de estados tal que, las trayectorias del sistema a lazo cerrado que empiezan en $M$, permanecen en $M$ en un entorno de $t=0$. Además, la correspondiente salida es idénticamente nula en ese intervalo de tiempo.

Para hallar la subvariedad invariante maximal por realimentación de estados contenida en $h^{-1}(0)$ aplicaremos el algoritmo de la Sección 5.3 al sistema (5.39) con la restricción algebraica

$$
h(x)=0 ;
$$

de este modo se hallarán la realimentación $u^{*}=\alpha^{*}(x)$ y la variedad $\mu^{*}$, subvariedad maximal anuladora de la salida para el sistema (5.39)-(5.40) realimentado con $u^{*}=\alpha^{*}(x)$.

Observación: el sistema

$$
\dot{x}=f^{*}(x),
$$

con $f^{*}(x)=f(x)+g(x) \alpha^{*}(x)$ y $x \in \mu^{*}$, identifica el comportamiento interno dinámico inducido sobre el sistema cuando la salida ha sido forzada, mediante una elección adecuada del estado inicial y de la entrada, a permanecer nula por algún intervalo de tiempo, es decir, la dinámica cero del sistema, [37]. 
Para determinar la dinámica cero del sistema (5.39)-(5.40), localmente alrededor de $x_{0}$, se supone usualmente que el sistema tiene algún grado relativo vectorial $\left\{r_{1}, \ldots, r_{m}\right\}$ en $x_{0}$, es decir que:

1. $L_{g_{j}} L_{f}^{k} h_{i}(x)=0$, para todo $1 \leq j \leq m, k<r_{i}-1,1 \leq i \leq m$, y para todo $x$ en un entorno de $x_{0}$;

2. la matriz de dimensión $m \times m$,

$$
A(x)=\left[\begin{array}{ccc}
L g_{1} L_{f}^{r_{1}-1} h_{1}(x) & \ldots & L g_{m} L_{f}^{r_{1}-1} h_{1}(x) \\
\vdots & & \vdots \\
L g_{1} L_{f}^{r_{m}-1} h_{m}(x) & \ldots & L g_{m} L_{f}^{r_{m}-1} h_{m}(x)
\end{array}\right]
$$

es no singular en $x_{0}$.

La existencia de grado relativo vectorial en $x_{0}$ permite definir un cambio local de coordenadas y obtener una representación especial del sistema llamada forma normal, a partir de la cual se obtiene de manera sencilla la dinámica cero. Sin embargo, aplicando el algoritmo de la Sección 5.3, podemos hallar la dinámica cero aún sin tener grado relativo vectorial y trabajando en las coordenadas originales. Para ilustrar esto analizaremos un caso particular.

\section{Ejemplo}

Consideramos el sistema

$$
\begin{aligned}
& \dot{x}_{1}=u_{1} \\
& \dot{x}_{2}=x_{4}+x_{3} u_{1} \\
& \dot{x}_{3}=x_{3}+x_{4} \\
& \dot{x}_{4}=u_{2}
\end{aligned}
$$


con salidas,

$$
\begin{aligned}
& h_{1}(x)=x_{1} \\
& h_{2}(x)=x_{2}
\end{aligned}
$$

Queremos hallar la variedad invariante maximal por realimentación de estados contenida en $h^{-1}(0)$ siendo $h(x)=\left[\begin{array}{ll}x_{1} & x_{2}\end{array}\right]^{\prime}$. Para eso aplicamos el algoritmo de la Sección 5.3 al sistema (5.43)-(5.46) considerando la restricción algebraica

$$
k^{1}(x)=h(x)=0 .
$$

Llamamos

$$
f(x)=\left[\begin{array}{c}
0 \\
x_{4} \\
x_{3}+x_{4} \\
0
\end{array}\right]
$$

y

$$
g_{1}(x)=\left[\begin{array}{c}
1 \\
x_{3} \\
0 \\
0
\end{array}\right], \quad g_{2}(x)=\left[\begin{array}{l}
0 \\
0 \\
0 \\
1
\end{array}\right]
$$

entonces

$$
L_{g} k^{1}(x)=\left[\begin{array}{ll}
L_{g_{1}} h_{1} & L_{g_{2}} h_{1} \\
L_{g_{1}} h_{2} & L_{g_{2}} h_{2}
\end{array}\right]=\left[\begin{array}{cc}
1 & 0 \\
x_{3} & 0
\end{array}\right]
$$

tiene rango 1 para todo $x$; además el sistema no tiene grado relativo vectorial en ningún punto. Mediante cálculos sencillos se tiene que, si

$$
E_{1}(x)=\left[\begin{array}{cc}
1 & 0 \\
-x_{3} & 1
\end{array}\right]
$$

resulta,

$$
\begin{gathered}
E_{1}(x)\left[L_{f} k^{1}(x)+L_{g} k^{1}(x)\right]= \\
=\left[\begin{array}{c}
\bar{k}^{2}(x) \\
k^{2}(x)
\end{array}\right]+\left[\begin{array}{c}
\bar{l}^{2}(x) \\
0
\end{array}\right] u=\left[\begin{array}{c}
0 \\
x_{4}
\end{array}\right]+\left[\begin{array}{ll}
1 & 0 \\
0 & 0
\end{array}\right] u
\end{gathered}
$$


luego,

$$
L_{f} k^{2}(x)=0 \quad \text { y } L_{g} k^{2}(x)=\left[\begin{array}{ll}
0 & 1
\end{array}\right] .
$$

Además, de la expresión

$$
\left[\begin{array}{c}
\bar{k}^{2}(x) \\
L_{f} k^{2}(x)
\end{array}\right]+\left[\begin{array}{c}
\bar{l}^{2}(x) \\
L_{g} k^{2}(x)
\end{array}\right] u=\left[\begin{array}{l}
0 \\
0
\end{array}\right]
$$

se obtiene $u^{*}=0$. Luego, el sistema

$$
\begin{aligned}
\dot{x} & =f^{*}(x) \\
h_{1}(x) & =x_{1} \\
h_{2}(x) & =x_{2}
\end{aligned}
$$

con $f^{*}(x)=f(x)$, tiene como variedad maximal anuladora de la salida a

$$
\begin{aligned}
\mu^{*}= & \left\{x \in R^{4}: k^{1}(x)=0, k^{2}(x)=0\right\}= \\
& =\left\{x \in R^{4}: x_{1}=x_{2}=x_{4}=0\right\} .
\end{aligned}
$$

El sistema (5.43)-(5.46) cuando $u=u^{*}=0 \mathrm{es,}$

$$
\begin{aligned}
& \dot{x}_{1}=0 \\
& \dot{x}_{2}=x_{4} \\
& \dot{x}_{3}=x_{3}+x_{4} \\
& \dot{x}_{4}=0
\end{aligned}
$$

Entonces, si tomamos condiciones iniciales en $\mu^{*}$ se tiene que $x_{1}(t)=x_{2}(t)=$ $x_{4}(t)=0$, para todo $t$. Luego,

$$
\dot{x}_{3}=x_{3}
$$

es la dinámica cero del sistema. 


\section{Capítulo 6}

\section{Conclusiones}

En este trabajo hemos estudiado una clase particular de sistemas diferenciales algebraicos: los semiexplícitos. Los hemos considerado desde el punto de vista geométrico, es decir como campos vectoriales sobre cierta variedad diferencial. De este modo la existencia y unicidad de solución para un SDAS puede inferirse a partir de la teoría de existencia y unicidad de solución para campos vectoriales.

En la Sección 6.1 detallaremos en forma precisa los aportes del trabajo. En la Sección 6.2 enunciaremos algunas posibles líneas de investigación en base a las ideas generadas en los Capítulos 3, 4 y 5 .

\subsection{Resumen de las contribuciones}

1. Hemos desarrollado un algoritmo que permite describir la familia de variedades restringidas, campo vectorial correspondiente y espacio de configuración asociados a un SDAS. Hemos considerado los casos de índice 1, 2 y 3, para luego pasar al caso general de índice $s$. Entonces, es posible asegurar la existencia y unicidad de solución para un SDAS a partir del estudio de las soluciones de su campo vectorial co- 
rrespondiente. Además, el dominio de este campo vectorial (espacio de configuración) representa un conjunto de condiciones iniciales posibles para el SDAS para asegurar existencia y unicidad de solución (condiciones iniciales consistentes).

2. Considerando un SPS no-standard, hemos considerado el sistema reducido correspondiente (que resulta un SDAS). Suponiendo que el sistema reducido es de índice 2 , hemos obtenido un resultado de aproximación de trayectorias para el SPS a partir de las trayectorias del sistema reducido.

Las hipótesis fundamentales para obtener este resultado fueron dos:

(i) Asegurar existencia y unicidad de solución para el sistema reducido. El estudio de las soluciones de este sistema se realizó sobre la base del análisis hecho en el Capítulo 3.

(ii) Suponer la estabilidad del equilibrio del sistema rápido (que en este caso, por la naturaleza del problema, resulta con matriz jacobiana singular). Esta estabilidad se garantizó utilizando la Teoría de la Variedad Centro.

3. Considerando un sistema no-lineal, afín en el control y con restricciones algebraicas, se ha resuelto el problema de hallar la variedad invariante maximal por realimentación de estados incluida en la superficie definida por las restricciones algebraicas.

Se ha considerado al sistema no-lineal junto con las restricciones algebraicas como un SDAS, donde las variables de control fueron tomadas como variables algebraicas. Se obtuvo un algoritmo para determinar tal variedad invariante. Se analizaron los casos de sistemas con un con- 
trol y con múltiples controles.

Como aplicación de lo anterior, se ha considerado un sistema no-lineal con control y con salida, y se ha resuelto el problema de hallar la subvariedad maximal por realimentación de estados anuladora de la salida. Este procedimiento permite describir la dinámica cero del sistema, aún en los casos en que no exista grado relativo vectorial.

\subsection{Futuras investigaciones}

A partir de los resultados obtenidos en los capítulos 2, 3 y 4, podemos enumerar algunas posibles futuras líneas de investigación.

1. Considerando el SDAS de ecuaciones (3.1)-(3.2) con el que se ha trabajado en el Capítulo 3, se plantea el problema de hallar sus soluciones en el contexto de órbitas de familias de campos, [38]. Este análisis generalizaría el estudio de soluciones realizado en el Capítulo 3 para sistemas regulares, al caso de sistemas no-regulares. Se puede plantear el problema de construir una distribución $K$ en $\mathbb{R}^{n+m}$, de modo tal que las soluciones del sistema sean curvas contenidas en las hojas integrales maximales de $K$.

2. Considerando un SPS no-standard con control, hallar una realimentación de estados de modo tal que el sistema realimentado resulte estable. En la Teoría de Perturbación Singular que analiza el caso standard [39], la estabilidad de los sistemas reducido y rápido son las hipótesis básicas para garantizar la estabilidad del sistema perturbado. Luego, considerando el caso no-standard estudiado en el Capítulo 4, 
se debería analizar bajo que condiciones el sistema reducido (en este caso un SDAS de índice 2) y el sistema rápido son estabilizables.

3. En la Teoría de Control por Modos Deslizantes, se trata de hallar una superficie ideal de deslizamiento para las trayectorias del sistema. Para que tal superficie exista se deben garantizar ciertas condiciones, [40]. Sin embargo, cuando estas condiciones no se cumplen, sería posible hallar una superficie ideal de deslizamiento (aunque de dimensión menor) a partir de los resultados obtenidos en el Capítulo 5. 


\section{Apéndice A}

\section{Teoría de la Variedad Centro}

Consideremos un sistema no-lineal

$$
\dot{x}=F(x)
$$

donde $F \in C^{r}(r \geq 0)$ está definido en un abierto $U \subset \mathbb{R}^{n}$, y sea $x_{0}$ un punto de equilibrio para $F$, es decir, $F\left(x_{0}\right)=0$. Sin pérdida de generalidad supondremos que $x_{0}=0$. Es bien conocido que la estabilidad asintótica local del equilibrio puede determinarse a partir del comportamiento de la aproximación lineal de $F$ en $x=0$. Luego, si llamamos

$$
J=\left(\frac{\partial F}{\partial x}\right)_{x=0}
$$

entonces,

(i) si todos los autovalores de $J$ están en el semiplano complejo izquierdo, $x=0$ es un punto de equilibrio asintóticamente estable de (A.1),

(ii) si uno o más autovalores de $J$ están en el semiplano complejo derecho, entonces $x=0$ es un punto de equilibrio inestable de (A.1).

Este resultado, conocido como Principio de Estabilidad de la Primera Aproximación, no cubre el análisis de la estabilidad local del equilibrio en el caso 
crítico, es decir cuando $J$ tiene algún autovalor con parte real 0 .

En este Apéndice describiremos algunos resultados que forman parte de la Teoría de la Variedad Centro, [29], y que resultan útiles en los casos críticos para el análisis de la estabilidad asintótica.

Definición A-1: Una subvariedad $C^{r}, S \subset U$, se dice localmente invariante para (A.1) si, para cada $x_{0} \in S$, existen $t_{1}<0<t_{2}$ tal que la curva integral $x(t)$ de (A.1) que satisface $x(0)=x_{0}$ es tal que $x(t) \in S, \forall t \in\left(t_{1}, t_{2}\right)$.

En adelante trabajaremos con un sistema de la forma

$$
\begin{aligned}
& \dot{x}=A x+f(x, y) \\
& \dot{y}=B y+g(x, y)
\end{aligned}
$$

con $x \in \mathbb{R}^{n}, y \in \mathbb{R}^{m}$ y $A$ y $B$ matrices constantes tales que todos los autovalores de $A$ tienen parte real 0 y todos los autovalores de $B$ tienen parte real negativa. Supondremos además que

$$
f(0,0)=0, g(0,0)=0, \quad\left(\frac{\partial f}{\partial(x, y)}\right)=0, \quad\left(\frac{\partial g}{\partial(x, y)}\right)=0 ;
$$

donde los dos jacobianos anteriores están evaluados en $(x, y)=(0,0)$.

Definición A-2: $y=h(x)$ variedad invariante para (A.2)-(A.3) se llama variedad centro si $h(0)=0$ y $\frac{\partial h}{\partial x}(0)=0$.

Observación: Si en (A.2)-(A.3), $f$ y $g$ son idénticamente nulos, entonces el sistema tiene dos variedades invariantes, $x=0$ e $y=0$. La variedad $y=0$ es la variedad centro; luego, todas las soluciones de (A.2)-(A.3) tienden exponencialmente rápido, cuando $t \rightarrow \infty$, a las soluciones de

$$
\dot{x}=A x
$$


Es decir, la ecuación sobre la variedad centro determina el comportamiento de las soluciones sobre el sistema completo. Este resultado puede extenderse cuando $f$ y $g$ son no nulos.

Teorema A-1: Existe una variedad centro $y=h(x)$ para (A.2)-(A.3), con $\|x\|<\delta$ y $h \in C^{2}$.

El flujo sobre la variedad centro está gobernado por el sistema $n$-dimensional

$$
\dot{u}=A u+f(u, h(u))
$$

El lema siguiente describe las propiedades de estabilidad de la variedad centro.

Lema: Sea $(x(t), y(t))$ una solución de (A.2)-(A.3) con $\|(x(0), y(0))\|$ suficientemente pequeño. Entonces existen constantes positivas $c_{1}$ y $\mu$ tales que,

$$
\|y(t)-h(x(t))\| \leq c_{1} e^{-\mu t}\|y(0)-h(x(0))\|, \quad \forall t \geq 0
$$

Por último, el siguente resultado establece que (A.4) tiene toda la información necesaria para determinar el comportamiento asintótico de soluciones de (A.2)-(A.3).

\section{Teorema A-2:}

(a) Supongamos que la solución nula de (A.4) es estable (asintóticamente estable) (inestable). Entonces la solución nula de (A.2)-(A.3) es estable (asintóticamente estable) (inestable).

(b) Supongamos que la solución nula de (A.4) es estable. Sea $(x(t), y(t))$ una solución de (A.2)-(A.3) con $(x(0), y(0))$ suficientemente pequeño. En- 
tonces existe una solución $u(t)$ de (A.4) tal que, cuando $t \rightarrow \infty$,

$$
\begin{aligned}
& x(t)=u(t)+O\left(e^{-\gamma t}\right) \\
& y(t)=h(u(t))+O\left(e^{-\gamma t}\right)
\end{aligned}
$$

con $\gamma>0$ constante. 


\section{Bibliografía}

[1] B. Haggman and P. Bryant, "Solutions of Singular Constrained Differential Equations: A Generalization of Circuits Containing CapacitorOnly Loops and Inductor-Only Cutsets," IEEE Trans. Circ. Syst. 31, 1015-1029 (1984).

[2] D. Hill and I. Mareels, "Stability Theory for Differential/Algebraic Systems with Application to Power Systems," IEEE Trans. Circ. Syst. 37, 1416-1423 (1990).

[3] W. Blajer, "A Projection Method Approach to Constrained Dynamic Analysis," J. Appl. Mechanics 59, 643-649 (1992).

[4] K.E. Brenan, S.L. Campbell and L.R. Petzold, Numerical Solution of Initial-Value Problems in Differential-Algebraic Equations, CLlassics in Applied Mathematics, SIAM, Philadelphia (1997).

[5] A. Kumar and P. Daoutidis, "A DAE Framework for Modeling and Control of Reactive Distillation Columns," Preprints of 4 th IFAC Symp. on Dynamics and Control of Chemical Reactors, Distillation Columns and Batch Processes, Helsingor, Denmark, 99-104 (1995). 
[6] C. Gear, "Simultaneous numerical solution of differential-algebraic equations," IEEE Trans. Circuit Theory 18, 89-95 (1971).

[7] L. Petzold, "Differential/algebraic equations are not ODE's," SIAM J. Sci. Statist. Comput. 3, 367-384 (1982).

[8] G. Soderlind, "DASP3-A Program for the Numerical Integration of Partitioned Stiff ODE's and Differential Algebraic Systems," Tech. Report TRITA-NA-8008, Dept. of Numer. Anal. and Comp. Science, The Royal Inst. of Technology, Stockholm, Sweden (1980).

[9] C. Gear and L. Petzold, "ODE Methods for the Solution of Differential/Algebraic Systems," Tech. Report 82-1103, Dept. of Comp. Sci., Univ. of Illinois at Urbana-Champaign (1982).

[10] W. Rheinboldt, "Differential-Algebraic Systems of Differential Equations on Manifolds," Math. of Comput. 43, 473-482 (1984).

[11] S. Campbell, Singular Systems of Differential Equations, Pitman Publishing Co., New York (1980).

[12] S. Campbell, Singular Systems of Differential Equations II, Pitman Publishing Co., New York (1982).

[13] L. Dai, Singular Control Systems, Lectures Notes in Control and Information Sciences, Springer Verlag, Heidelberg (1989).

[14] C. Gear, B. Leimkuhler and G. Gupta, "Automatic integration of Euler-Lagrange equations with constraints," J. Comput. Appl. Math. 12/13, 77-90 (1985). 
[15] H. Krishnan and H. McClamroch, "Tracking in Nonlinear DifferentialAlgebraic Control Systems with Applications to Constrained Robot Systems," Automatica 30, 1885-1897 (1994).

[16] R. Abraham, J. Marsden and T. Ratiu, Manifolds, Tensor Analysis, and Applications, Addison-Wesley Publishing Co., Massachusetts, (1983).

[17] S. Reich, "On a Geometrical Interpretation of Differential-Algebraic Equations," Circ. Syst. Sign. Proc. 9, 367-382 (1990).

[18] S. Reich, "On an Existence and Uniqueness Theory for Nonlinear Differential Algebraic Equations," Circ. Syst. Sign. Proc. 10, 343-359 (1991)

[19] A. Szatkowski, "Geometric Characterization of Singular Differential Algebraic Equations," Int. J. Syst. Sci. 23, 167-186 (1992).

[20] P. Rabier and W. Rheinboldt, "A Geometric Treatment of Implicit Differential-Algebraic Equations," J. of Diff. Eq. 109, 110-146 (1994).

[21] M. Etchechoury y C. Muravchik, "Regularidad de Sistemas Diferenciales Algebraicos Semiexplícitos," Proceedings de la VI Reunión en Procesamiento de la Información y Control RPIC97, San Juan, Argentina; 1, 58-63 (1997).

[22] E. Coddington and N. Levinson, Theory of Ordinary Differential Equations, McGraw-Hill Publishing Co., New Delhi (1977). 
[23] H. McClamroch and H. Krishnan, "Non-standard singularly perturbed control systems and differential-algebraic equations," Int. J. Control 55, 1239-1253 (1992).

[24] H. Krishnan and H. McClamroch, "On the Connection Between Nonlinear Differential-Algebraic Equations and Singularly Perturbed Control Systems in Nonstandard Form," IEEE Trans. Aut. Cont. 39, 10791084 (1994).

[25] H. Khalil, "Feedback Control of Nonstandard Singularly Perturbed Systems," IEEE Trans. Aut. Cont. 34, 1052-1060 (1989).

[26] A. Kumar and P. Daoutidis, Control of nonlinear differential algebraic equation systems, Chapman and Hall/CRC, London (1999).

[27] M. Etchechoury and C. Muravchik, "Singular Perturbation Models and Differential Algebraic Equations," Lat. Amer. Appl. Res. 29, 205212 (1999).

[28] M. Etchechoury and C. Muravchik, "Nonstandard Singularly Perturbed Systems and Differential Algebraic Systems of Higher Index," aceptado para su publicación en Applied Mathematics and Computation, julio 2001.

[29] J. Carr, Applications of Centre Manifold Theory, Springer-Verlag, New York Inc. (1981).

[30] H. Khalil, Nonlinear Systems, Macmillan Publishing Company, New York (1992). 
[31] M. Corless and L. Glielmo, "On the Exponential Stability of Singularly Perturbed Systems", SIAM J. Cont. and Opt. 30, 1338-1360 (1990).

[32] V. Butuzov, A. Vasil'eva and M. Fedoryuk, Asymptotic Methods in the Theory of Ordinary Differential Equations, in Progress in Mathematics (R. Gamkrelidze, Ed.), Plenum Press (1970).

[33] A. De Luca, "Control properties of robot arms with joint elasticity", Proc. Math. Theory Networks and Systems (1987).

[34] A. De Luca, A. Isidori and F. Nicolao, "An application of nonlinear model matching to the dynamic control of robot arm with elastic joints," Proc. IFAC Symposium on Robot Control, Barcelona, 55-62 (1985).

[35] C.W. Gear, "Maintaining solution invariants in the numerical solution of ODEs," SIAM J. Sci. Statist. Comput. 7, 734-743 (1986).

[36] M. Etchechoury y C. Muravchik, "Sistema Diferencial-Algebraico con Control: Subvariedad Invariante por Realimentación," aceptado para ser presentado en la IX Reunión de Procesamiento de la Información y Control, a realizarse en la UNL, Santa Fe, septiembre 2001.

[37] A. Isidori, Nonlinear Control Systems: An Introduction, Springer Verlag, Berlin (1995).

[38] H. Sussmann, "Orbits of Families of Vector Fields and Integrability of Distributions," Trans. of AMS, 180, 171-188 (1973).

[39] P. Kokotovic, H. Khalil and J. O'Reilly, Singular Perturbation Methods in Control, Academic Press, New York (1986). 
[40] H. Sira Ramírez, "Differential geometric methods in variable-structure control," Int. J. Control 48, 1359-1390 (1988). 